\title{
ZINC ION FLUXES ON THE PATHWAY TO MAMMALIAN SPERM FERTILIZATION COMPETENCY
}

A Dissertation
presented to
the Faculty of the Graduate School
at the University of Missouri-Columbia
In Partial Fulfillment
of the Requirements for the Degree
Doctor of Philosophy
KARL KERNS
Dr. Peter Sutovsky, Dissertation Supervisor
May 2019


(C) Copyright by Karl Kerns 2019

All Rights Reserved 
The undersigned, appointed by the dean of the Graduate School, have examined the dissertation entitled

\section{ZINC ION FLUXES ON THE PATHWAY TO MAMMALIAN SPERM FERTILIZATION COMPETENCY}

presented by Karl Kerns, a candidate for the degree of Doctor of Philosophy and hereby certify that, in their opinion, it is worthy of acceptance.

Professor Peter Sutovsky

Professor Randall Prather

Professor Timothy Safranski

Professor Michael Smith

Professor Tim Evans 


\section{ACKNOWLEDGEMENTS}

I would like to express my enormous gratitude to my advisor, Dr. Peter Sutovsky, for giving me the opportunity to join his laboratory at the University of Missouri. I am very thankful to be under his mentorship as well as for his guidance and support not only academically but also in life itself. His insight and intuition in sperm biology is second to none.

I would like to thank my committee members, Dr. Randall Prather, Dr. Timothy Safranski, Dr. Michael Smith, and Dr. Tim Evans for their guidance, aiding in critical thinking and intellectual growth. I am thankful for their inspiration and spark of awe and admiration for that which remains unknown in the reproductive biology field.

I cannot thank members of the Sutovsky Lab and our collaborators enough. First, to Miriam Sutovsky for originally training me in techniques, ordering supplies, and lab management, as well as for her friendship and encouragement throughout the years. To Dr. Michal Zigo for his technical expertise, assisting in projects, and our regular conversations. Thank you to Dalen Zuidema for assisting in sperm studies requiring oocytes. I am appreciative of Sutovsky Lab past, Dr. Wonhee Song, for her moral support. Collaborators that made work in this thesis possible include Dr. Erma Drobnis for our human sperm studies; Dr. Richard Oko, Wei Xu, and Lauren Hamilton for zymography studies; Dr. David Miller and Momal Sharif for oviductal glycan studies; and Patricio Morales for co-authoring my first review manuscript.

Thank you to Melissa Samuel, Sabrina Hammond, and everyone involved at the National Swine Resource and Research Center for providing routine boar semen 
samples and tissue samples. Much of the work done here would not have been possible without fresh, on site boar semen. Also thank you to Carmen Heinzmann and Carter Zeisneiss of International Boar Semen for donated boar semen and industry advice throughout the years. Likewise, for routine conversation with $\mathrm{Dr}$. Brad Belstra, Dr. Steve Terlouw, and Amanda Minton for swine industry perspectives.

I would like to thank the University of Missouri Division of Animal Sciences as well as the founding members of Food for the $21^{\text {st }}$ Century (F21C) for the world premier environment they have provided and continue to provide for graduate student training in the area of reproductive biology.

Last, but definitely not least, I would like to thank my parents, Steve and Becky Kerns, for their continued support of my graduate studies and feedback. None of this would have been possible if they had not instilled a passion for the swine industry and genetic progress through boar semen technologies. Likewise, I am thankful for my Redemption's Hill Church family. I am thankful to the Lord above for all this mentioned here, His graces and mercies, and for unveiling a small part of His creation's design at the moments leading up to the formation of new life. 


\section{TABLE OF CONTENTS}

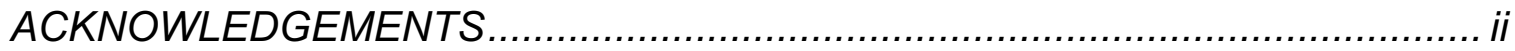

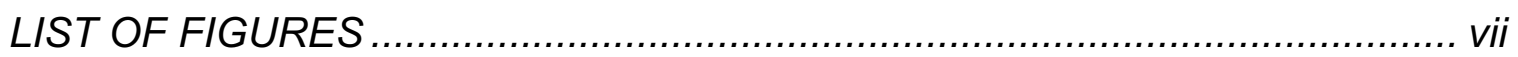

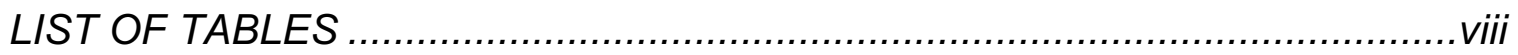

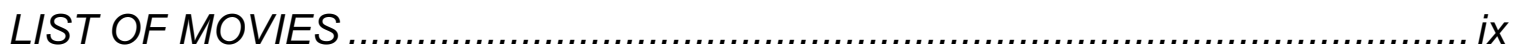

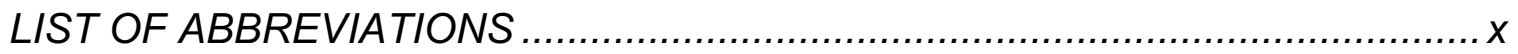

LIST OF OFFICIAL GENE SYMBOLS AND NAMES ...............................

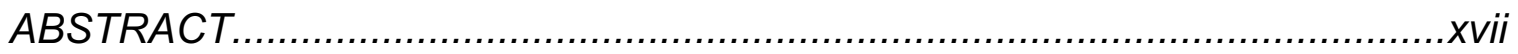

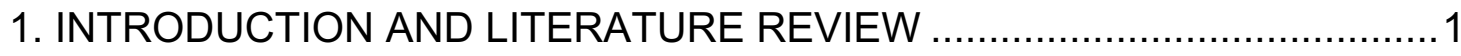

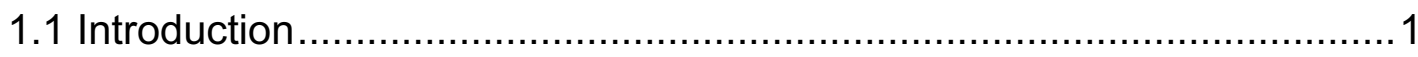

1.2 Zinc in Biological Systems Overview ........................................

1.3 Zinc-interacting Sperm Proteins ............................................... 5

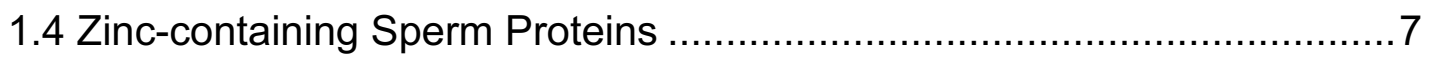

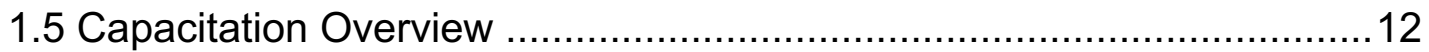

1.6 Zinc as a Regulator of Sperm Capacitation and Fertilization ................15

1.7 Ubiquitin Proteasome System Overview ................................... 19

1.8 The 20S Proteasome During Sperm Capacitation ...........................20

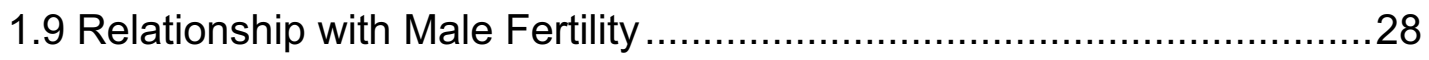

1.10 Areas for Future Exploration .................................................. 34

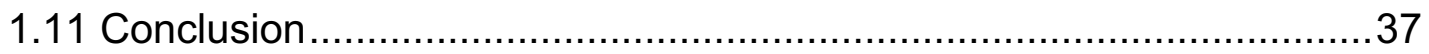


1.12 Acknowledgements

2. PART 1: CHARACTERIZING ZINC ION DISTRUBUTION IN MAMMALIAN

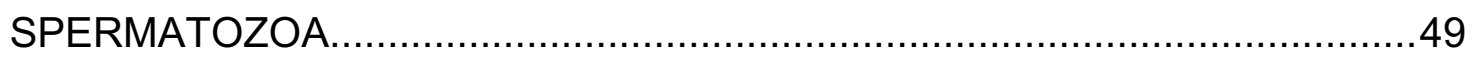

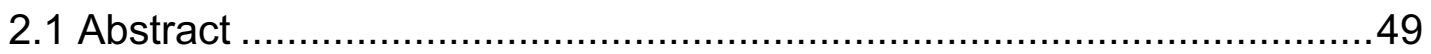

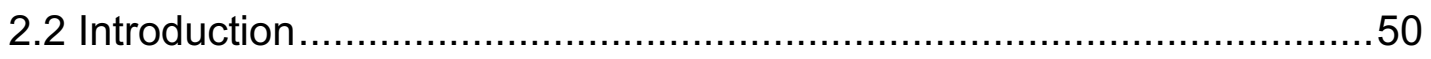

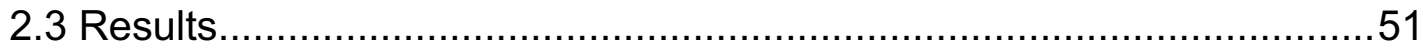

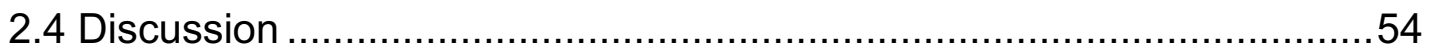

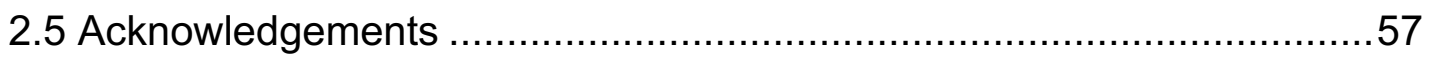

3. PART 2: ZINC ION FLUX DURING MAMMALIAN SPERM CAPACITATION

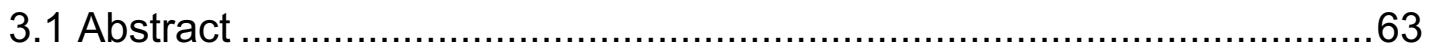

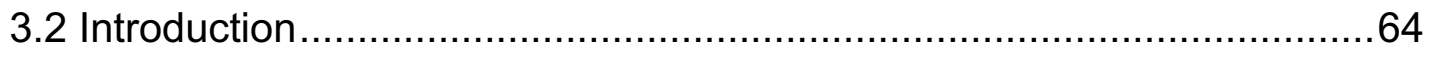

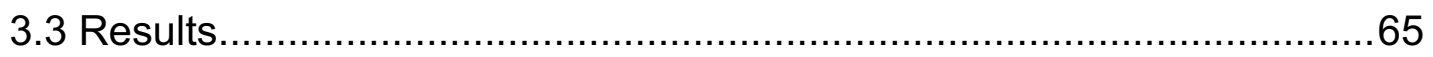

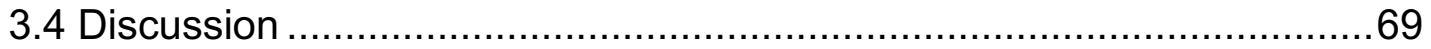

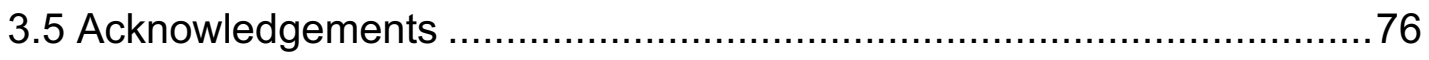

4. BOAR EJACULATE SEQUENCE ENDOWS SPERM ZINC SIGNATURE SUBPOPULATIONS AND CAPACITATION-INDUCED ZINC EFFLUX IS NECESSARY FOR OVIDUCTAL SPERM RESERVOIR GLYCAN RELEASE \& ACTIVATION OF ZONA PELLUCIDA PROTEINASE MMP2 _.....................92

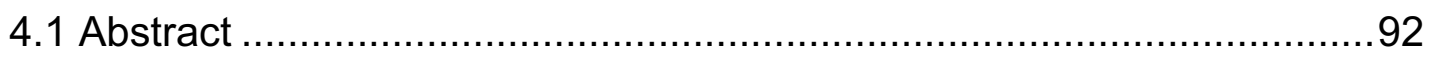

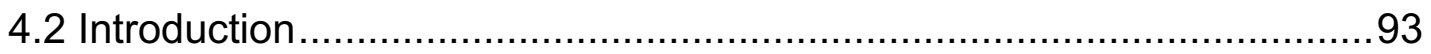

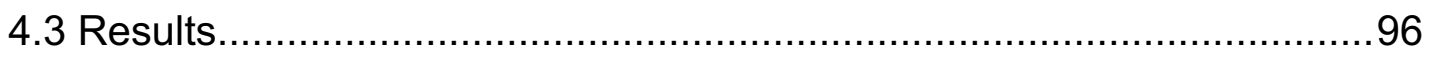

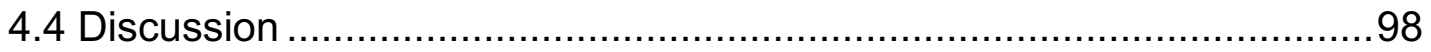




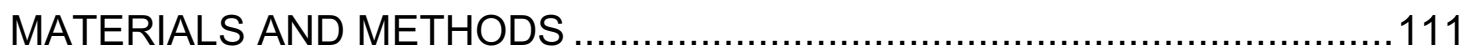

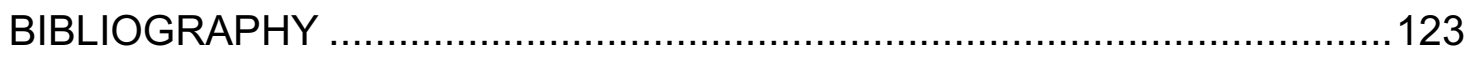

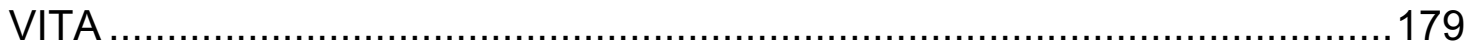




\section{LIST OF FIGURES}

Figure

1.1 $\mathrm{Zn}^{2+}$ regulation of mammalian spermatozoa fertilization competency summary

1.2 Summary of $26 \mathrm{~S}$ proteasome-regulated events of sperm capacitation ...... 42

2.1 Zinc content of the developing boar spermatozoa prior to ejaculation........58

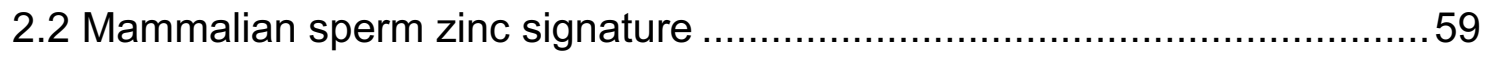

2.3 Super resolution confocal microscopy of boar sperm zinc signature ..........60

2.4 Bull sperm head Zn-efflux, reflected by transition from signature 2 to 3 , is associated with a posterior-to-anterior nuclear membrane modification wave.

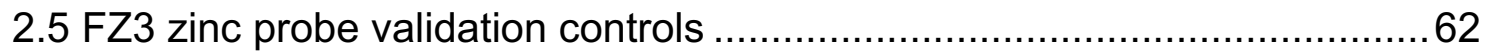

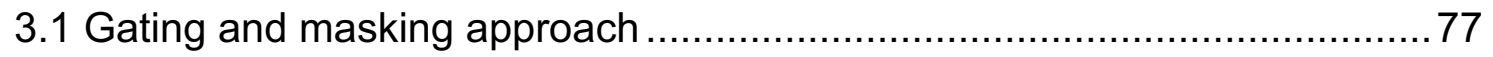

3.2 Capacitation status confirmation under high versus low bicarbonate IVC

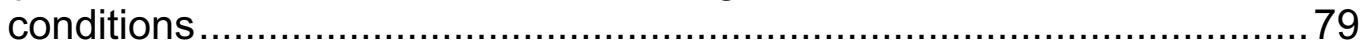

3.3 Acrosomal status and membrane integrity of zinc signature .....................81

3.4 Modulation of internal and external $\mathrm{Zn}^{2+}$ during IVC ..............................83

3.5 Zinc signature of human spermatozoa after IVC …................................. 84

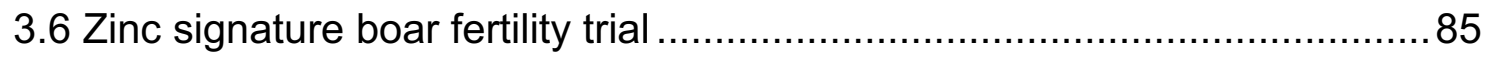

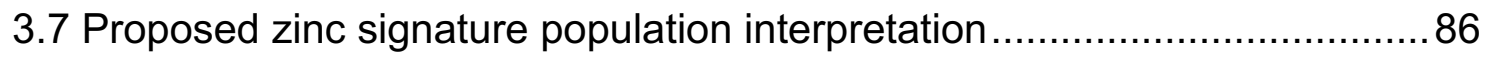

4.1 Boar sperm zinc signature differs by ejaculate fraction ......................... 105

4.2 BTS + $1.0 \mathrm{mM} \mathrm{ZnCl} 2$ prevents spontaneous sperm tyrosine phosphorylation $(\mathrm{pY})$, an indicator of sperm capacitation ..........................................106

4.3 MU Vanguard extender prevents storage-induced sperm capacitation. ... 107

4.4 Zinc inhibits $\mathrm{P}_{4}$-induced sperm release from oviductal glycans ............... 108 


\section{LIST OF TABLES}

Table

1.1 General summary of testicular/sperm Zn-containing and interacting proteins.

1.2 Compilation of proteins that may interact with the sperm proteasome during capacitation/fertilization.

3.1 Statistical analysis of zinc signature and acrosomal status ....................88

3.2 Effect of proteasomal inhibition on zinc signature ................................. 89

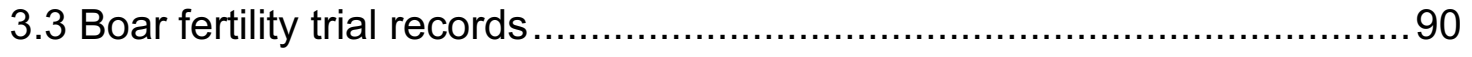

4.1 Boar ejaculate fraction endows sperm zinc signature subpopulations ....110

4.2 MU Vanguard extender prevents storage-induced sperm capacitation... 110 


\section{LIST OF MOVIES}

Table

Page

3.1 Comparison of sperm motility under varied IVC conditions ......................90

3.2 Hyperactivated spermatozoa have zinc signature 2 .............................90

3.3 Zinc signature in motile vs. immotile spermatozoa after IVC ....................91

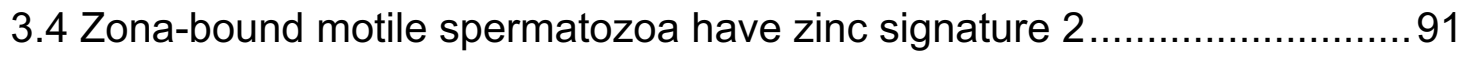




\section{LIST OF ABBREVIATIONS}

$\begin{array}{ll}\text { Al } & \text { Artificial insemination } \\ { }^{\circ} \mathrm{C} & \text { Degrees Celsius } \\ \text { ACE } & \text { Angiotensin converting enzyme } \\ \text { ADAM } & \text { A disintegrin and metalloproteinase } \\ \text { AE } & \text { Acrosomal exocytosis } \\ \text { AF647 } & \text { Alexa Fluor }{ }^{\text {TM }} \text { 647 } \\ \text { ALP } & \text { Alkaline phosphatase } \\ \text { AQN/AQN1 } & \text { Spermadhesin AQN1 } \\ \text { ATP } & \text { Adenosine triphosphate } \\ \text { AWN/AWN3 } & \text { Spermadhesin AWN3 } \\ \text { bi-SiaLN } & \text { Biantennary 6-sialylated lactosamine oligosaccharide } \\ \text { BSP } & \text { Binder of sperm } \\ \text { BTS } & \text { Beltsville Thaw Solution [extender] } \\ \text { Ca }{ }^{2+} & \text { Calcium ion } \\ \text { CaCl } 2 & \text { Calcium dichloride } \\ \text { CAMP } & \text { Cyclic adenosine monophosphate } \\ \text { CLBL } & \text { Clasto-Lactacystin } \beta \text {-Lactone } \\ \text { CO } 2 & \text { Carbon dioxide } \\ \text { ddH2O } & \text { Double-distilled water } \\ \text { DEDTC } & \text { N,N-diethyldithiocarbamate } \\ \text { DMPS } & 2,3 \text {-dimercaptopropane-1-sulfonate } \\ \text { DMSA } & \text { (2R,3S)-2,3-Bis(sulfanyl)butanedioic acid } \\ & \end{array}$




\begin{tabular}{|c|c|}
\hline DMSO & Dimethyl sulfoxide \\
\hline DNA & Deoxyribonucleic acid \\
\hline EDTA & 2,2',2",2"'-(Ethane-1,2-diyldinitrilo)tetraacetic acid \\
\hline EPOX & Epoxomicin \\
\hline FN1 & Fibronectin \\
\hline FONDECYT & Fondo Nacional de Desarrollo Científico y Tecnológico \\
\hline FZ3 & FluoZin ${ }^{T M}-3$ AM \\
\hline g & Gram \\
\hline GFP & Green fluorescent protein \\
\hline GLM & General Linear Model \\
\hline GM6001 & GM6001 MMP inhibitor \\
\hline GPCR & G-protein-coupled receptor \\
\hline H33342 & Hoechst 33342 \\
\hline $\mathrm{HCO}_{3}^{-}$ & Bicarbonate \\
\hline HeLa & Henrietta Lacks cells \\
\hline HEPES & $\begin{array}{l}\text { 4-(2-Hydroxyethyl)piperazine-1-ethanesulfonic acid, } \\
\mathrm{N} \text {-(2-Hydroxyethyl)piperazine-N'-(2-ethanesulfonic acid) }\end{array}$ \\
\hline HVCN & Hydrogen voltage gated channel 1 \\
\hline IAM & Inner acrosomal membrane \\
\hline IBFC & Image-based flow cytometry \\
\hline ICSI & Intracytoplasmic sperm injection \\
\hline $\lg \mathrm{A}$ & Immunoglobulin A \\
\hline $\lg G$ & Immunoglobulin G \\
\hline
\end{tabular}




\begin{tabular}{ll} 
IgM & Immunoglobulin M \\
IRB & Internal Review Board \\
IUI & Intrauterine insemination \\
IVC & in vitro capacitation \\
IVF & in vitro fertilization \\
LDH & Lactate dehydrogenase \\
mg & Milligram \\
MG132 & N-benzyloxycarbonyl-L-leucyl-L-leucyl-L-leucinal \\
MgCl 2 & Magnesium dichloride \\
mL & Milliliter \\
mM & Millimolar \\
MMP & Matrix metalloproteinase \\
MT & Metallothionein \\
mTALP & Modified Tyrode's albumin lactate pyruvate \\
MU & University of Missouri \\
NIFA & National Institute of Food and Agriculture \\
NIH & National Institutes of Health \\
nM & Nanomolar \\
NP-40 & Nonidet P-40 \\
ODF & Outer dense fibers \\
PAGE & Progestrone \\
PBS & Polyacrylamide gel electrophoresis \\
\hline
\end{tabular}




\begin{tabular}{ll} 
PEB1 & Phosphatidylethanolamine binding protein 1 \\
PI & propidium iodide \\
PI- & propidium iodide negative \\
PI+ & propidium iodide positive \\
PI3K & Phosphoinositide 3-kinase \\
PI3K & Phosphoinositide 3-kinase \\
PKA & Protein kinase A \\
PKA & Protein kinase A \\
PMFAB & Plasma membrane fatty acid binding protein \\
PNA & Lectin Arachis hypogea/peanut agglutinin \\
PNA+ & PNA positive (modified acrosome) \\
PPi & Inorganic pyrophosphate \\
PSP-1 & Spermadhesin PSP-1 \\
PTK & Protein tyrosine kinase \\
PTP & Protein tyrosine phosphatase \\
PTP & Protein tyrosine phosphatase \\
pY & Protein tyrosine phosphorylation \\
PYR-41 & 4[4-(5-nitro-furan-2-ylmethylene)-3,5-dioxo-pyrazolidin- \\
ROS & Reactive oxygen species \\
RP & Regulatory complex/particle \\
SACY & Sperm-soluble adenylyl cyclase \\
SAS & Statistical analysis system \\
\hline
\end{tabular}




\begin{tabular}{|c|c|}
\hline SDS & Sodium dodecyl sulfate \\
\hline SOD & Superoxide dismutase \\
\hline STED & Stimulated emission depletion \\
\hline suLe $e^{x}$ & 3-O-sulfated Lewis X trisaccharide \\
\hline SVS2 & Seminal vesicle secretory protein 2 \\
\hline TL-HEPES & Tyrode's lactate HEPES buffer \\
\hline TPEN & $N, N, N^{\prime}, N^{\prime}$ 'tetrakis(2-pyridinylmethyl)-1,2-ethanediamine \\
\hline $\operatorname{TrX}$ & Triton $\mathrm{X}-100$ \\
\hline Tyr & Tyrosine \\
\hline UPS & Ubiquitin proteasome system \\
\hline USDA & U.S. Department of Agriculture \\
\hline ZIP & Zrt- and Irt-like protein \\
\hline ZIP & Zrt-, Irt-like protein \\
\hline $\mathrm{Zn}$ & Zinc \\
\hline $\mathrm{Zn}^{2+}$ & Zinc ion \\
\hline ZnBP & Zinc-binding proteins \\
\hline $\mathrm{ZnCl}_{2}$ & Zinc dichloride \\
\hline $\mathrm{ZnR}$ & Zn-sensing receptor \\
\hline $\mathrm{ZnT}$ & Zinc transporter \\
\hline ZP & Zona pellucida \\
\hline$\mu \mathrm{L}$ & Microliter \\
\hline$\mu \mathrm{M}$ & Micromolar \\
\hline$\omega$ & Omega \\
\hline
\end{tabular}




\section{LIST OF OFFICIAL GENE SYMBOLS AND NAMES}

ADAM

AKAP3

AKAP8

ACRBP

ADRM1

ALP

ACE

BSP3

CatSper

SOD1

EGFR

GPR39

GPCR

HVCN1

JAMM

LDH-X

$\mathrm{LDH}-\mathrm{C}_{4}$

MMP2

MMP9

MFGE8

SOD2
A disintegrin and metalloproteinase

A-kinase anchoring protein 3

A-kinase anchoring protein 8

Acrosin binding protein

Adhesion regulating molecule 1

Alkaline phosphatase

Angiotensin converting enzyme

Binder of sperm 3

Cation channels of sperm

Cytosolic dimeric $\mathrm{Cu} / \mathrm{Zn}-\mathrm{SOD}$

Epidermal growth factor receptor

G Protein-coupled receptor 39

G-protein-coupled receptor

Hydrogen voltage gated channel 1

JAB1/MPN/Mov34 metalloenzyme

Lactate dehydrogenase isoenzyme

Lactate dehydrogenase isoenzyme

Matrix metallopeptidase 2

Matrix metallopeptidase 9

Milk fat globule-EGF factor 8 protein

Mitochondrial matrix Mn-SOD

NYD-SP27/PLCZ1 phospholipase C zeta 1 isoform 


\begin{tabular}{|c|c|}
\hline PRM2 & Protamine 2 \\
\hline PSMD1 & Proteasome $26 \mathrm{~S}$ subunit, non-ATPase 1 \\
\hline PSMD14 & Proteasome $26 \mathrm{~S}$ subunit, non-ATPase 14 \\
\hline PSMD4 & Proteasome $26 \mathrm{~S}$ subunit, non-ATPase 4 \\
\hline PSMD6 & Proteasome $26 \mathrm{~S}$ subunit, non-ATPase 6 \\
\hline PSMD7 & Proteasome $26 \mathrm{~S}$ subunit, non-ATPase 7 \\
\hline PSMD8 & Proteasome $26 \mathrm{~S}$ subunit, non-ATPase 8 \\
\hline PSMA1 & Proteasome subunit alpha 1 \\
\hline PSMA2 & Proteasome Subunit alpha 2 \\
\hline PSMA4 & Proteasome Subunit alpha 4 \\
\hline PSMB1 & Proteasome Subunit beta 1 \\
\hline PSMB10 & Proteasome Subunit beta 10 \\
\hline PSMB6 & Proteasome Subunit beta 6 \\
\hline EC-SOD/SOD3 & Secretory tetrameric extracellular SOD \\
\hline SPINK2 & Serine peptidase inhibitor, Kazal type 2 \\
\hline SERPINE2 & Serpin family $\mathrm{E}$ member 2 \\
\hline sNHE & Sperm-specific $\mathrm{Na}^{+} / \mathrm{H}^{+}$exchanger \\
\hline UBR7 & Ubiquitin protein ligase $\mathrm{E} 3$ component $\mathrm{N}$-recognin 7 \\
\hline UBA1 & Ubiquitin-activating enzyme E1 \\
\hline VCP & Valosin-containing protein \\
\hline ZP3 & Zona pellucida 3 glycoprotein \\
\hline
\end{tabular}




\title{
ZINC ION FLUXES ON THE PATHWAY TO MAMMALIAN SPERM FERTILIZATION COMPETENCY
}

\author{
Karl Kerns \\ Dr. Peter Sutovsky, Dissertation Supervisor
}

\begin{abstract}
For decades, the role of the divalent ion calcium was the focus of understanding mechanisms leading to sperm fertilization competency and management of semen storage. Little focus has been placed on other divalent ions, including zinc ion $\left(\mathrm{Zn}^{2+}\right)$. Further, the ultimate maturation event preparing mammalian spermatozoa for fertilization, sperm capacitation, was first described in 1951, yet its regulatory mechanisms remain poorly understood. Here, we document a novel biological phenomenon of a unique $\mathrm{Zn}^{2+}$ distribution (further zinc signature) associated with mammalian spermatozoa from the round spermatid stage of spermiogenesis, to epididymal maturation, ejaculation, and up to 72 hours of liquid semen storage. Using image-based flow cytometry (IBFC), we identified four distinct sperm zinc signatures present in boar, bull, and human spermatozoa. The zinc signature was altered after sperm capacitation, reduced by proteasomal inhibitors, removed by zinc chelation, and maintained with addition of external $\mathrm{ZnCl}_{2}$. The zinc signature differed between the three major boar ejaculate fractions. These differences set in sperm ejaculatory sequence likely establish two major sperm cohorts; one destined for populating the sperm oviductal reservoir and the other that is capable of fertilizing mature, ovulated oocytes at the time of mating/insemination. Management of the sperm zinc signature prevented spontaneous, pathological capacitation, by day 3 of extended liquid boar semen storage and could allow for
\end{abstract}


use of fewer sperm per artificial insemination (Al) dose to increase the usage of high genetic value sires. A newly formulated semen extender was able to mimic qualities of the pre-sperm rich fraction. On a subcellular level, the capacitation induced $\mathrm{Zn}^{2+}$ efflux allows for release from oviductal glycans studied with the oviductal epithelium mimicking glycan binding assay. Sperm $\mathrm{Zn}^{2+}$ efflux also activates zinc-containing enzymes involved in sperm penetration of the zona pellucida, such as the inner acrosomal membrane metalloproteinase MMP2 that had a severely reduced activity in the presence of $\mathrm{Zn}^{2+}$ by gel zymography. In context of the fertilization-induced oocyte zinc spark and the ensuing, oocyteissued polyspermy-blocking zinc shield, the inhibitory effect of $\mathrm{Zn}^{2+}$ on spermborne enzymes may contribute to the fast block of polyspermy. Altogether, our findings establish a new paradigm on the role of $\mathrm{Zn}^{2+}$ in sperm function, paving the way for improved semen analysis, in vitro fertilization (IVF) and the optimization of Al and semen distribution. 


\section{CHAPTER 1}

\section{INTRODUCTION AND LITERATURE REVIEW}

\section{Published as part of:}

Kerns K, Zigo M, Sutovsky P. 2018. Zinc: A Necessary lon for Mammalian Sperm Fertilization Competency. International Journal of Molecular Sciences, 19, 4097.

\section{DOI:10.3390/iims19124097}

and

Kerns K, Morales P, Sutovsky P. 2016. Regulation of Sperm Capacitation by the 26S Proteasome: An Emerging New Paradigm in Spermatology. Biology of Reproduction, 94:5, 117, 1-9. DOI:10.1095/biolreprod.115.136622

\subsection{Introduction}

The present review focuses on two major components, the role of zinc and the ubiquitin proteasome system, and their roles in mammalian sperm capacitation. The mechanism of sperm capacitation remains among a large knowledge gap in the field of fertilization and early development. Despite decades of intense research, the mechanisms that convey fertilizing competency to spermatozoa traveling through the female reproductive system are not well understood (De Jonge, 2017). In fact, leaders in sperm capacitation research have recently disproven their own hallmark discoveries which were long accepted paradigms and theories [particularly the role of protein tyrosine phosphorylation, covered throughout (Alvau et al., 2016)]. 


\subsection{Zinc in Biological Systems Overview}

Zinc $(\mathrm{Zn})$ is one of the highly abundant elements on earth, an essential micronutrient to all things living, typically occurring as a divalent cation metal with moderate reactivity and reducing properties. Essential biological roles of zinc include signaling, enzymatic activities, regulation of normal growth and sexual maturation, digestion, homeostasis of central nervous system and mitochondrial oxidative stress (Lee, 2018b; Levaot and Hershfinkel, 2018). Conversely, zinc imbalance or altered zinc-signaling accompanies pathologies including but not limited to Alzheimer's disease (Lovell, 2009; Szewczyk, 2013; Prakash et al., 2015), blindness, cancer, digestive ailments, growth retardation and inflammation (Prasad, 2009). While ancient Etruscans and Romans may have already recognized medicinal properties of zinc salts (Giachi et al., 2013), its biological importance was only fully realized in the $19^{\text {th }}$ century, and entered mainstream human medicine a hundred years later, when the first studies were conducted on dwarfism, human zinc deficiency, and general importance of zinc as a growth factor (Prasad, 2013).

Cells of all organisms ranging from $E$. coli to mammals tightly regulate free zinc ion $\left(\mathrm{Zn}^{2+}\right)$ distribution, even though its toxicity is relatively low (Beyersmann and Haase, 2001; Outten and O'Halloran, 2001). In humans, nearly $90 \%$ of $\mathrm{Zn}^{2+}$ is found in the muscle and bone (Wastney et al., 1986). Other organs containing significant concentrations of $\mathrm{Zn}^{2+}$ are the prostate, liver, gastrointestinal tract, kidney, skin, lung, brain, heart and pancreas (Llobet et al., 1988; Bentley and 
Grubb, 1991; He et al., 1991). Homeostasis of $\mathrm{Zn}^{2+}$ is important for survival and fitness; thus, when $\mathrm{Zn}^{2+}$ is consumed in excess, it is important for the body to handle its surplus (Jones et al., 1979). Upon ingestion and absorption through the small intestine, the redistribution of $\mathrm{Zn}^{2+}$ occurs via the serum, where $\mathrm{Zn}^{2+}$ is bound predominantly to albumin (major binding protein for up to $60 \%$ of $\mathrm{Zn}^{2+}$ ); the remaining $\mathrm{Zn}^{2+}$ is bound predominantly to 12 other proteins including $\alpha_{2-}$ macroglobulin, transferrin, ceruloplasmin, $\lg G$, $\lg \mathrm{A}$, $\lg \mathrm{M}$, complement C4, haptoglobin, and prealbumin (Prasad and Oberleas, 1970; Scott and Bradwell, 1983). Serum $Z^{2+}$ accounts for only $\sim 0.1 \%$ of bodily $Z n^{2+}$ (Rukgauer et al., 1997). Further, there is no known specialized $\mathrm{Zn}^{2+}$ storage system in the body, and therefore only the daily intake of $\mathrm{Zn}^{2+}$ will ensure steady availability (Scott and Bradwell, 1983).

On the cellular level, $30-40 \%$ of $\mathrm{Zn}^{2+}$ localizes in the nucleus, while $50 \%$ is stored in the cytoplasm and the rest is associated with membranes (Vallee and Falchuk, 1993). There are two families of proteins that are responsible for the movement of $\mathrm{Zn}^{2+}$ through biological membranes, thus exercising sustained homeostatic control. These include zinc-importer (ZIP; Zrt-, Irt-like) family proteins that transport $\mathrm{Zn}^{2+}$ into the cytosol and the zinc transporter ( $\mathrm{ZnT}$ ) family proteins transporting $\mathrm{Zn}^{2+}$ out of the cytosol (Lichten and Cousins, 2009). Completion of human genome sequencing identified 14 members of ZIP (designated ZIP1-14) and 10 members ZnT (designated ZnT1-10) (Lichten and Cousins, 2009) families. Few studies have inspected major tissues for expression patterns of ZnTs in humans (Foresta et al., 
2014), and the expression of ZIPs during spermatogenesis is only known in mice (Croxford et al., 2011). Once $\mathrm{Zn}^{2+}$ enters a cell by ZnTs and ZIPs, it becomes sequestered within the endoplasmic reticulum, mitochondria and Golgi, or other cell type-specific membrane bound vesicular structures, also called zincomsomes (Zalewski et al., 1993; Wellenreuther et al., 2009). Cytosolic $\mathrm{Zn}^{2+}$ complexing with cytosolic proteins maintains the concentration of free cytosolic $\mathrm{Zn}^{2+}$ within range between picomoles and nanomoles, depending on the cell type (Sensi et al., 1997; Outten and O'Halloran, 2001; Vinkenborg et al., 2009; Qin et al., 2011). Up to $20 \%$ of cytosolic $\mathrm{Zn}^{2+}$ is bound by the apoprotein thionein, to form metallothionein (MT). MTs are small ubiquitous proteins (6-7 kDa), rich in cysteine that can complex transition metal ions (Coyle et al., 2002; Tapiero and Tew, 2003). One molecule of MT can bind up to seven $\mathrm{Zn}^{2+}$, buffering excess $\mathrm{Zn}^{2+}$ and supplying such cation under $\mathrm{Zn}^{2+}$ deficiency states (Maret and Krezel, 2007; Blindauer and Leszczyszyn, 2010).

Limited information exists on the regulation of $\mathrm{Zn}^{2+}$ homeostasis in reproductive system. In female gametes, $\mathrm{Zn}^{2+}$ plays a gatekeeping role in regulating meiotic resumption (Kim et al., 2010; Kim et al., 2011; Zhao et al., 2014). A novel phenomenon of $\mathrm{Zn}^{2+}$ release from the mammalian oocyte at fertilization was recently reported (Kim et al., 2011; Que et al., 2017), inspiring some of the work on male gametes that will be discussed throughout later chapters. The importance of $\mathrm{Zn}^{2+}$ for male fertility only emerged recently, propelled in part by consumer interest in nutritional supplements containing ionic trace minerals. 


\subsection{Zinc-interacting Sperm Proteins}

In eel, zinc is necessary for the sustenance of germ cells and spermatogenesis progression, with $N, N, N^{\prime}, N^{\prime}$-tetrakis(2-pyridinylmethyl)-1,2-ethanediamine (TPEN) -induced Zn deficiency causing germ cell apoptosis (Yamaguchi et al., 2009). Zinc transporters have been examined in rat, specifically MT I \& II in spermatocytes (Elgazar et al., 2005) as well as tesmin, a testis-specific metallothionein-like protein (Sugihara et al., 1999). Further, ZnT-7 in mouse testis may supply spermatogenesis-required zinc (Chi et al., 2009). $\mathrm{Zn}^{2+}$ transporter ZIP9 serves as a membrane-associated receptor interacting with G-protein Gna11 to mediate nonclassical testosterone signaling cascade in murine spermatogenic GC-2 cells (Shihan et al., 2015). Further, the spontaneous $\mathrm{Ca}^{2+}$ oscillations observed in spermatogenic cells seem to be modulated by $\mathrm{Zn}^{2+}$ (Lopez-Gonzalez et al., 2016). Zinc ions begin to colonize spermatogenic cells during the final stages of spermatid differentiation when they are incorporated in the nucleus (Barney et al., 1968) and nascent outer dense fibers (ODF) (Bedford and Calvin, 1974; Baccetti et al., 1976). Additional $\mathrm{Zn}^{2+}$ is incorporated into the nucleus at ejaculation (Bjorndahl et al., 1986). Nuclear $\mathrm{Zn}^{2+}$ associates with protamines and forms zinc bridges, most likely through imidazole groups of histidine and thiols of cysteine (Porath et al., 1975), proposed by Björndahl and Kvist to stabilize the sperm chromatin structure (Bjorndahl and Kvist, 2010, 2011). These authors showed that a rapid sperm chromatin decondensation can be induced by $\mathrm{Zn}^{2+}$ chelation with $2,2^{\prime}, 2^{\prime \prime}, 2^{\prime \prime \prime}-$ (Ethane-1,2-diyldinitrilo)tetraacetic acid (EDTA), causing the disruption of the

protamine zinc bridges (Kvist, 1980a, b; Roomans et al., 1982). In the sperm 
flagellum, $\mathrm{Zn}^{2+}$ is bound to sulfhydryl groups of ODF protein cysteine groups, to protect the nascent flagellum from premature oxidation (Henkel et al., 2001). During epididymal transit, $\mathrm{Zn}^{2+}$ is selectively removed from the flagellum by a 160 $\mathrm{kDa}$ protein, enabling oxidation of sulfhydryl groups and stiffening the ODF to support progressive motility (Henkel et al., 2001). High concentrations of $\mathrm{Zn}^{2+}$ have been found in the acrosome (Andrews et al., 1994) and proteolytic conversion of proacrosin to acrosin is inhibited by $\mathrm{Zn}^{2+}$ (Johnsen et al., 1982; Steven et al., 1982), as it probably involves $\mathrm{Zn}$-dependent metalloproteinases. Zinc ions also associate with sperm membranes, where they interact with lipoproteins and membranebound metalloproteins in which they react with sulfhydryl groups of cysteine and therefore fulfill a membrane stabilizing function (Bettger and O'Dell, 1981; Mankad et al., 2006). Active removal of $\mathrm{Zn}^{2+}$ is therefore a prerequisite for the completion of sperm capacitation (Andrews et al., 1994), a complex structural and molecular remodeling event that endows spermatozoa within female reproductive tract with ability to fertilize. High concentrations of $\mathrm{Zn}^{2+}(100 \mu \mathrm{M})$ reduce sperm motility in a reversible manner (Riffo et al., 1992). Initiation of motility following ejaculation (Acott and Carr, 1984) and the increased motility of the capacitation-induced sperm hyperactivation are both dependent upon intracellular alkalinization (Ho et al., 2002).

Additional $\mathrm{Zn}^{2+}$ becomes incorporated into spermatozoa during ejaculation (Bjorndahl et al., 1986; Foresta et al., 2014) where it is believed to have protective function in terms of sperm chromatin decondensation (Bjorndahl and Kvist, 2010, 
2011), sperm motility and metabolic inhibition (Babcock et al., 1983; Riffo et al., 1992), membrane stabilization (Bettger and O'Dell, 1981) and antioxidant activity (Bray and Bettger, 1990; Narasimhaiah et al., 2018). As $\mathrm{Zn}^{2+}$ becomes incorporated into spermatozoa upon mixing with seminal fluid, there are also seminal fluid $\mathrm{Zn}$-interacting proteins competitively binding free $\mathrm{Zn}^{2+}$. In humans, a bulk of seminal fluid $\mathrm{Zn}^{2+}$ is bound to high and low molecular weight ligands derived from prostatic and vesicular secretions (Arver, 1980, 1982; Arver and Eliasson, 1982; Siciliano et al., 2000). Among them, semenogelins participate in the formation of coagulum, to prevent retrograde flow of semen deposited in the female tract. Prostasomes, small, exosome-like lipoprotein vesicles, are the main zinc-binding partners in human seminal fluid (Robert and Gagnon, 1999; Vivacqua et al., 2004). Zinc-binding proteins have also been found in seminal fluid of boar (Mogielnicka-Brzozowska et al., 2011) and dog (Johnson et al., 1969; MogielnickaBrzozowska et al., 2015), and designated as ZnBP1-6.

\subsection{Zinc-containing Sperm Proteins}

Zinc-containing proteins, commonly known as metalloproteins, are capable of binding one or more $\mathrm{Zn}^{2+}$, usually as a requirement for their biological activity.

Human genome sequencing and combined proteomic approaches independently identified 1,684 proteins in the human proteome as zinc-containing proteins (Andreini et al., 2006). Metalloproteins can be further divided into three groups, i.e. i) metalloenzymes, ii) metallothioneins, and iii) gene regulatory proteins (Coleman, 1992; Vallee and Falchuk, 1993). Metallothioneins have been discussed in the 
previous section. Gene regulatory proteins are nucleoproteins directly involved with replication and transcription of DNA. Such DNA binding proteins can be further categorized into three structurally distinct groups, containing: i) zinc fingers, ii) zinc clusters, or iii) zinc twists (Vallee et al., 1991). Spermatozoa may have limited use for gene regulatory proteins since they are transcriptionally silent; however, these proteins, as for instance protamine P2 (discussed earlier), are used heavily for DNA condensation, packaging, and transcriptional suppression (Bianchi et al., 1992). Majority of this section will therefore be dedicated to zinc-containing metalloenzymes, which play a vital role in sperm function.

More than 300 enzymes have been identified that require $\mathrm{Zn}^{2+}$ for their function (Vallee and Falchuk, 1993), representing more than 50 different enzyme types. $\mathrm{Zn}^{2+}$ is the only metal that is encountered in all six classes of enzymes, (i.e. oxidoreductases, transferases, hydrolases, lyases, isomerases and ligases). This can be attributed to two properties of $\mathrm{Zn}^{2+}:$ i) relatively low toxicity when compared to other transition metals (Seiler and Sigel, 1988), and ii) stable association and coordination flexibility with macromolecules (Vallee and Auld, 1990). $\mathrm{Zn}^{2+}$ fulfills three functions in the Zn-enzymes: i) catalytic, ii) co-active (co-catalytic), and iii) structural (Vallee and Auld, 1992). Catalytic $\mathrm{Zn}^{2+}$ takes part directly in enzyme catalysis. Co-active $\mathrm{Zn}^{2+}$ enhances or diminishes catalytic function in conjunction with catalytic $\mathrm{Zn}^{2+}$, but is not indispensable for catalytic function (Vallee and Auld, 1992). Structural $\mathrm{Zn}^{2+}$ is required for stabilization of quaternary structure of oligomeric enzymes. 
Matrix metalloproteinases (MMPs) belong to a family of zinc-dependent endopeptidases, involved in the degradation of extracellular matrix proteins. Since the first discovery of MMPs in the early 1960s, the number of known MMPs have grown and at least 28 species have been identified to this date; for subtype categorization, distribution and substrate specificities, see review by Cui et al. (2017). Structurally, a typical MMP contains a propeptide, a catalytic metalloproteinase domain, a linker peptide (hinge region) and a hemopexin domain (Cui et al., 2017). The catalytic domain contains two $\mathrm{Zn}^{2+}$ (catalytic and structural) and up to three calcium ions $\left(\mathrm{Ca}^{2+}\right)$ which stabilize the structure. The cysteine rich region in propeptide chelates the catalytic $\mathrm{Zn}^{2+}$, keeping MMPs in an inactive zymogen form (Nagase et al., 2006). MMP2 and MMP9, also referred to as Gelatinase-A and Gelatinase-B were described in human seminal fluid (Shimokawa Ki et al., 2002; Tentes et al., 2007) and canine epididymal fluid and seminal fluid (Saengsoi et al., 2011; Warinrak et al., 2015). Furthermore, MMP2 was found to be localized in acrosomal and tail region of normal morphological ejaculated human and canine spermatozoa, while MMP9 was localized in the tail region (Buchman-Shaked et al., 2002; Warinrak et al., 2015). High levels of MMP2 are associated with high (70\%) motility and significantly elevated levels of MMP9 are observed in semen samples with low sperm count (Warinrak et al., 2015). Ferrer at al. (2012b) demonstrated that MMP2 together with acrosin were confined to the inner acrosomal membrane of epididymal bull sperm and thus introducing the possibility of their cooperation in enzymatic digestion of the oocyte zona pellucida (ZP) during penetration. Regulation of said MMPs by zinc ion fluxes 
associated with sperm capacitation should be investigated. Kratz et al. (2016) demonstrated that the levels of seminal MMP2 and MMP9 are correlated with oxidative stress in men, making this a potential diagnostic tool for semen quality/male infertility. Finally, Atabakhsh et al., (Atabakhsh et al., 2018) noticed a positive correlation between seminal fluid MMP2 activity and sperm count, as well as fertilization and embryo quality in couples undergoing assisted reproductive therapy (ART) by intracytoplasmic sperm injection (ICSI), offering a potential predictor of ICSI outcome.

Superoxide dismutases (SOD) are metalloenzymes responsible for dismutating the superoxide anion $\left(\mathrm{O}_{2}^{-}\right)$, to hydrogen peroxide $\left(\mathrm{H}_{2} \mathrm{O}_{2}\right)$ and oxygen $\left(\mathrm{O}_{2}\right)(\mathrm{McCord}$ and Fridovich, 1969). Three isoforms have been reported in mammals; i) the cytosolic dimeric $\mathrm{Cu} / \mathrm{Zn}-\mathrm{SOD}$ (SOD1), ii) the mitochondrial matrix Mn-SOD (SOD2), and the secretory tetrameric extracellular SOD (EC-SOD/SOD3) (Marklund, 1984). It has been shown that both seminal fluid and spermatozoa contain SOD activity (Alvarez et al., 1987; Beconi et al., 1993; O'Flaherty et al., 1997; Peeker et al., 1997; Marti et al., 2007; Kowalowka et al., 2008; Strzezek and Fraser, 2009; Park et al., 2012), of which $75 \%$ was attributed to SOD1, which is also the main SOD isoform in spermatozoa SOD. The SOD activity in spermatozoa is several-fold higher than SOD activity levels previously measured in more than 50 different human somatic cell types (Peeker et al., 1997). O'Flaherty et al. (O'Flaherty et al., 1997) suggested an important role of superoxide anion in sperm hyperactivation and capacitation; therefore, adequate balance between 
superoxide radical generation and dismutation is vital for proper function of spermatozoa as implicated by Sikka (Sikka, 2001).

Another significant group of $\mathrm{Zn}^{2+}$ containing proteins of spermatozoa are sorbitol dehydrogenases that convert sorbitol to fructose and endow spermatozoa with and have been correlated to motility (Dhanda et al., 1981). Lactate dehydrogenase isoenzyme ( $\left.\mathrm{LDH}-\mathrm{X}, \mathrm{LDH}-\mathrm{C}_{4}\right)$ also has been reported to have relationship with sperm motility (Wheat and Goldberg, 1983; Gavella and Cvitkovic, 1985; Virji, 1985). It was shown at least in mice that the inhibition of $\mathrm{LDH}-\mathrm{C}_{4}$ blocked sperm capacitation (Duan and Goldberg, 2003). We previously reported the presence of a ring finger ubiquitin ligase homologous to UBR7 in round spermatids and spermatozoa (Zimmerman et al., 2014), and implicated this zinc finger containing enzyme in spermiogenesis and possibly in the proteolytic degradation of the ZP at fertilization (Zimmerman et al., 2011). Angiotensin converting enzyme (ACE), yet another important $\mathrm{Zn}^{2+}$ containing protein, has been reported in testis, epididymis and spermatozoa of stallion, boar, and man (Jaiswal et al., 1984; Yotsumoto et al., 1984; Brentjens et al., 1986; Krassnigg et al., 1986; Vivet et al., 1987; Dobrinski et al., 1997; Gatti et al., 1999). Several roles in reproduction have been proposed for ACE, including spermatogenesis (Reeves and O'Dell, 1988), sperm capacitation (Singh et al., 1985; Kondoh et al., 2005), and sperm-ZP binding (Zigo et al., 2013). Alkaline phosphatase (ALP), a homodimeric enzyme containing two $\mathrm{Zn}^{2+}$ and one $\mathrm{Mg}^{2+}$ is present in mammalian seminal fluid (Bell and Lake, 1962; Einarsson et al., 1976) and spermatozoa (Soucek and Vary, 1984) Precise role of ALP in 
reproduction remains to be discovered, though it may serve as decapacitating factor (Bucci et al., 2014). Additional $\mathrm{Zn}^{2+}$ containing proteins found in the spermatozoa include fructose-bisphosphate aldolases (Gillis and Tamblyn, 1984) of which class-Il possesses $\mathrm{Zn}^{2+}$ (Mildvan et al., 1971), and alcohol dehydrogenase present in human testis and spermatozoa (Dafeldecker and Vallee, 1986; Khokha et al., 1994). The ADAM (a disintegrin and metalloproteinase) protein family plays a role in multiple events leading up to fertilization, including gamete migration and sperm reservoir interactions, spermoocyte ZP binding, and sperm-oocyte plasma membrane adhesion and fusion [see review (Cho, 2012)]. Noteworthy, proteins in this family possess metalloproteinase domains with $\mathrm{Zn}^{2+}$ (Chunghee, 2005). The metalloproteinase domain, however, is cleaved during epididymal transit and only the disintegrin domain remains in mature spermatozoon (Cho, 2012). Altogether, it is likely that the zinc-interacting proteome plays varied and often essential roles in the regulation of sperm homeostasis and fertilizing ability. Rather than a complete list of zinc-containing proteins, we focused on proteins that are well characterized. We are aware that there are many zinc-containing proteins to be characterized in spermatozoa.

\subsection{Capacitation Overview}

The acquisition of fertilizing potential by spermatozoa is attained in the epididymis, but the actual ability to fertilize is functionally suppressed until capacitation (Chang, 1951; Austin, 1952). Capacitation is a series of biochemical and biophysical alterations to the spermatozoa that increase intracellular $\mathrm{pH}$, increase fluidity of 
cell membranes, increase amplitude and pattern of motility, and initiate a complex signal transduction pathway including dramatic up-regulation of protein tyrosine phosphorylation (Visconti et al., 1995a; Visconti et al., 1995b). This essential (for fertilization) structural and functional remodeling prepares the spermatozoa for interaction with the cumulus-oocyte complex and to undergo acrosomal exocytosis (Gadella et al., 2008). It is believed capacitation can be delayed but is not fully reversible, and once initiated predestines spermatozoa towards cell death unless they reach the oocyte (Suarez, 2008).

It is important to consider how the in vivo environment might interact with spermsignaling in events initiating and during sperm capacitation. With that in mind, the oviductal sperm reservoir aids in reducing polyspermy and helps establish a sequential release of freshly capacitated spermatozoa. Spermatozoa bind to oviductal epithelial cells within the sperm reservoir located near the isthmus in most mammalian species. Species-specific carbohydrate residues on the oviductal cells and seminal plasma proteins coating sperm head surface (spermadhesins and binder-of-sperm proteins) mediate this binding and their release is modulated by the timing of ovulation [reviewed in (Miller, 2015; Suarez, 2015)]. The precise mechanism for sperm binding to and release from the reservoir is not well understood, but thought to correlate to sperm capacitation (Smith and Yanagimachi, 1991; Lefebvre and Suarez, 1996). Related hypotheses postulate that: 1) sperm release is coordinated with loss of the aforementioned sperm surface proteins by proteolysis and/or by shedding from plasma membrane (Demott and Suarez, 1992); 2) increase in spermatozoa motility to a 
hyperactivated state allows for release by shear force (Pacey et al., 1995); 3) carbohydrate residues on the epithelial cell surface are degraded by glycosidase enzymes present in oviductal fluid around the time of ovulation (Carrasco et al., 2008a; Carrasco et al., 2008b; Hung and Suarez, 2010); and 4) spermadhesins and other oviduct-secreted glycoproteins may compete with sperm binding of other oviductal carbohydrates (Song et al., 2010); however, additional follow up studies should be performed. These hypotheses are not mutually exclusive and a synergy between said mechanisms may exist.

The prevailing view attributes sperm release from the sperm reservoir to sperm capacitation. Capacitation begins with the removal of decapacitation factors. Candidate decapacitation factors include the spermadhesins AQN and AWN in the pig (Jonakova et al., 1998; Ekhlasi-Hundrieser et al., 2005), DF glycol protein (Fraser, 1998), phosphatidylethanolamine binding protein 1 (PEB1) (Gibbons et al., 2005; Nixon et al., 2006), sperm antigen 36, CRISP1, plasma membrane fatty acid binding protein (PMFAB) (Nixon et al., 2006), seminal vesicle secretory protein 2 (SVS2) (Kawano et al., 2014; Araki et al., 2015), and SERPINE2 (Lu et al., 2011) in the mouse, and NYD-SP27/PLCZ1 in the mouse and humans (Boatman and Robbins, 1991; Pastor-Soler et al., 2003). Some of these proteins are components of the seminal plasma fraction of semen (Jonakova et al., 1998), and others are of testicular origin and located on the outer acrosomal membrane. Removal of decapacitation factors triggers a cascade of events starting with an efflux of cholesterol from the sperm plasma membrane (Davis, 1981). Removal of 
cholesterol modulates an influx of bicarbonate and calcium that regulates the activity of sperm-soluble adenylyl cyclase (SACY) to increase intracellular cAMP [cyclic adenosine monophosphate (Boatman and Robbins, 1991; Pastor-Soler et al., 2003)]. An increase in cAMP activates protein kinase A (PKA), which phosphorylates protein tyrosine kinases (PTK) to an activated state and protein tyrosine phosphatases (PTP) to an inhibited state. This accelerates protein tyrosine phosphorylation in the sperm head and tail - a hallmark of sperm capacitation (Baker et al., 2006; Goto and Harayama, 2009).

\subsection{Zinc as a Regulator of Sperm Capacitation and Fertilization}

Zinc ions play a vital role in sperm capacitation, regulating key events responsible for fertilization competency (summarized in Fig. 1.1). Much as $\mathrm{Ca}^{2+}$ influx was understood as key for capacitation, today it is understood that $\mathrm{Zn}^{2+}$ efflux is the gatekeeper to this important $\mathrm{Ca}^{2+}$ influx (Loeb, 1914; Yanagimachi and Kanoh, 1953; Yanagimachi and Usui, 1974; Rogers and Yanagimachi, 1975). In the following discussion of sperm capacitation it is important to note the contrasting definitions of sperm capacitation (physiological vs. biochemical) (Gervasi and Visconti, 2016) and we will discuss it strictly from the earlier in its original definition [the acquisition of the capacity to fertilize (Chang, 1951)]. There has been a noticeable paucity of pivotal discoveries in sperm capacitation translatable from rodent models to humans (De Jonge, 2017). Much of this is criticized as a lack of in vivo or minimal inclusion of an in vitro female component in sperm capacitation studies; however, a critical review of literature suggests this could be due to subtle 
but vivid differences in the study models and/or experimental design. This includes species differences in attaining intracellular alkalinization (Florman et al., 2010) thus regulating $\mathrm{Ca}^{2+}$ entry [solely the $\mathrm{Na}^{+}$-dependent $\mathrm{Cl}^{-} / \mathrm{HCO}_{3}{ }^{-}$exchanger (Zeng et al., 1996) and possibly the sperm-specific $\mathrm{Na}^{+} / \mathrm{H}^{+}$exchanger sNHE (Wang et al., 2007) in murine; hydrogen voltage gated channel, HVCN1 in humans (Lishko et al., 2010)] as well as a result of using epididymal spermatozoa (as opposed to ejaculated). Both of these factors have a notable impact and result in studies that do not mimic the physiology of ejaculated human semen.

There is a moderate negative correlation between flagellar $\mathrm{Zn}^{2+}$ content, and sperm global and progressive motility in humans (Henkel et al., 1999). Chelation of sperm $\mathrm{Zn}^{2+}$ by $(2 R, 3 S)-2,3-B i s($ sulfanyl)butanedioic acid (DMSA), 2,3dimercaptopropane-1-sulfonate (DMPS), or DL-penicillamine leads to increased average straight line velocity and progressive sperm motility while decreasing the percentage of nonlinear motile spermatozoa (Wroblewski et al., 2003). Though discovered before the importance of HVCN1 in sperm motility activation and capacitation surfaced, previous authors believed this $\mathrm{Zn}^{2+}$ removal to be solely associated with stiffening of the ODF. Voltage-gated proton channel, HVCN1 localizes to the sperm flagellum and is responsible for sperm cytoplasmic alkalinization through transmembrane proton extrusion (Babcock et al., 1983). HVCN1 is asymmetrically positioned, likely providing differing alkalized microenvironments and gradients in relationship to the symmetrically positioned 
CatSper channels thereby responsible for asymmetrical flagellar bending during hyperactivation (Miller et al., 2018).

The relationship between 26S proteasome activity and $\mathrm{Zn}^{2+}$ is unclear, though the proteasomal regulatory subunit PSMD14/Rpn11 contains a metalloprotease-like $\mathrm{Zn}^{2+}$ site (Ambroggio et al., 2003). $\mathrm{Zn}^{2+}$ has been implicated in regulating proteasome-dependent proteolysis in HeLa cells (Kim et al., 2004). Contrarily to the high seminal fluid $\mathrm{Zn}^{2+}$ concentrations (2 mM) inhibiting HVCN1, lower concentrations (20 and $50 \mu \mathrm{M}$ ) have been implicated in promoting acrosomal exocytosis in sea urchin (Clapper et al., 1985) and bovine (Michailov et al., 2014) spermatozoa during in vitro capacitation. It is believed that $\mathrm{Zn}^{2+}$ interacts with $\mathrm{Zn}$ sensing receptor (ZnR) GPR39 of the G-protein-coupled receptor (GPCR) family found in the sperm acrosome. This interaction stimulates acrosomal exocytosis through epidermal growth factor receptor (EGFR) transactivation and phosphorylation of phosphoinositide 3-kinase (PI3K) causing acrosomal $\mathrm{Ca}^{2+}$ mobilization (Michailov et al., 2014). This implicates a multifaceted role of $\mathrm{Zn}^{2+}$ in sperm capacitation and therefore more research will be needed to fully comprehend these contrasting pathways (inhibiting vs. inducing acrosomal exocytosis).

Successful embryo development in mammals depends upon efficient antipolyspermy defense, preventing the entry of more than one spermatozoon in the oocyte cytoplasm at fertilization and thus alleviating an embryo-lethal polyploidy. 
While membrane depolarization and cortical granule exocytosis are regarded as the main barriers to polyspermy, a sperm-induced $\mathrm{Zn}^{2+}$ release from the oocyte cortex, nicknamed the $\mathrm{Zn}^{2+}$ spark, has recently been discovered in mammals (Kim et al., 2011; Que et al., 2017). Besides the oocyte $\mathrm{Zn}^{2+}$ spark (Kim et al., 2011), there is also a physiochemical $\mathrm{ZP}$ hardening and a $300 \% \mathrm{Zn}^{2+}$ increase in the $\mathrm{ZP}$ matrix observed when the fertilized oocyte zona becomes refractory to sperm binding in the mouse (Que et al., 2017). Such a proposed new anti-polyspermy defense mechanism is plausible, though the exact mechanism is not known. Further, $\mathrm{Zn}^{2+}$ is chemorepulsive, possibly overriding the chemoattraction of oocytesecreted progesterone in capacitated human, mouse, and rabbit spermatozoa (Guidobaldi et al., 2017). This signaling could in fact de-capacitate spermatozoa already bound to the zona or present in the perivitelline space at the time of fertilization. It is likely such hijacking sperm Zn-signaling and de-capacitation complements the blockage of fertilization through traditional anti-polyspermy mechanisms. $\mathrm{Zn}^{2+}$ has been shown to inhibit fertilization when added to bovine in vitro fertilization (IVF) media (Stephenson and Brackett, 1999). In further support of this mechanism, $\mathrm{Zn}^{2+}$ regulates the activity of the proposed sperm-borne $\mathrm{ZP}$ lysins, the proteinases (Ferrer et al., 2012b) implicated in ZP penetration, including acrosin (Johnsen et al., 1982; Steven et al., 1982), 26S proteasome [HeLa cells, (Kim et al., 2004); 20S proteasomal core, bovine brain (Amici et al., 2002)] and MMP2 [brain, (Backstrom et al., 1992)], therefore playing a regulatory role in sperm-ZP penetration. Additionally, inhibitors of zinc-dependent metalloproteases hinder sperm passage through the cumulus oophorus during porcine IVF (Beek et 
al., 2012). Altogether, a Zn-signaling polyspermy defense mechanism may exist from the zinc spark of newly fertilized oocytes and is explored in later Chapters.

\subsection{Ubiquitin Proteasome System Overview}

The conventional ubiquitin proteasome system (UPS) covalently modifies substrate proteins with multi-ubiquitin chains and targets them for degradation by the $26 \mathrm{~S}$ proteasome, a holoenzyme made up of a $19 \mathrm{~S}$ regulatory complex/particle (RP) and 20S core, each composed of multiple proteasomal subunit proteins. Both ATP dependent and non-ATP dependent subunits make up the 19S RP. Particular non-ATPase subunits, PSMD4 and ADRM1, recognize ubiquitinated proteins (Husnjak et al., 2008) and ATPase subunits rotate and regulate protein entrance into the $20 \mathrm{~S}$ core (Husnjak et al., 2008). Among the non-ATPase subunits, deubiquitinases PSMD7 and PSMD14 (Verma et al., 2002; Luise et al., 2011) release ubiquitin molecules/chains tagged to proteins, after which step the substrate protein can enter the $20 \mathrm{~S}$ core and be degraded (Husnjak et al., 2008).

Initial work on sperm proteasome was done in the ascidian (Saitoh et al., 1993), echinoderm (Yokota and Sawada, 2007), human (Morales et al., 2003) and porcine (Sutovsky et al., 2003; Sutovsky et al., 2004) models; however, since 2000 there has been a substantial amount of work undertaken in other mammals and even in birds and plants. The original ascidian studies showed that trypsin-like and chymotrypsin-like activities of sperm protease(s) were required for fertilization (Hoshi et al., 1981) because when these activities were inhibited, spermatozoa 
were unable to penetrate the vitelline coat of the oocyte (Sawada et al., 1984). Later, the trypsin-like enzymes involved in fertilization were identified as sperm acrosin and spermosin (Sawada et al., 1984), and the chymotrypsin-like enzymes were identified as sperm proteasome subunits (Kong et al., 2009). The research on extracellular UPS in mammalian gametes revealed that proteasomes have an essential role in reproduction, ranging from sperm quality control mechanisms in the epididymis (Sutovsky et al., 2004; Zimmerman et al., 2011; SaldívarHernández et al., 2015) to steps involved with sperm capacitation events (Kong et al., 2009) and assisting with sperm-zona pellucida (ZP) penetration (Sutovsky et al., 2004; Zimmerman et al., 2011; Saldívar-Hernández et al., 2015).

\subsection{The 20S Proteasome During Sperm Capacitation}

Proteasomal inhibitors MG132 and epoxomicin block the chymotrypsin-like activity of human sperm proteasome in a reversible fashion and significantly alter sperm capacitation in vitro (Kong et al., 2009). The chymotrypsin-like activity of the sperm proteasome is also reduced in the presence of the inhibitors of protein kinase A (PKA) and protein tyrosine kinases (PTK) while PKA activators have significantly increased the chymotrypsin-like activity of the sperm proteasome (Kong et al., 2009). These studies also showed that sperm capacitation is accompanied by massive phosphorylation at serine/threonine and tyrosine residues of several sperm proteasome subunits, and a slightly increased phosphorylation at serine residues. These findings imply that the sperm proteasome is involved in the capacitation process. Also implied is a relationship is between sperm PKA activity 
and proteasomal proteolysis. Once activated by PKA, the proteasome could directly or indirectly, modulate substrate protein phosphorylation at serine and threonine residues through a feedback loop; such a mechanism is presently under investigation by Sutovsky and Morales laboratories. Studies also suggest the proteasome may be involved in the degradation of threonine kinases during capacitation and of several proteins that undergo phosphorylation at threonine residues (Kong et al., 2009).

Regulating the sequestration and activity of sperm PKA, AKAP3 (Xu and Qi, 2014) is one of the compartmentalized A-kinase anchoring proteins (AKAPs) found in the sperm tail structures that also contain proteasomes (Mochida et al., 2000). Proteasome inhibition by MG132 prevented the PKA-induced proteasomal degradation of AKAP3 during bull sperm capacitation. However a related protein, AKAP8 (syn. AKAP-95) was not degraded, suggesting that AKAP3 degradation is likely to be specific and has a physiological importance in capacitation (Hillman et al., 2013). This finding also indicates that proteasomal activities towards AKAPs may be regulated by diverse mechanisms in the sperm tail and sperm head, where some AKAPs localize to the proteasome-rich acrosomal region (Vijayaraghavan et al., 1999). At present, it is not clear whether AKAP3 and other proteasometargeted AKAPs are constitutively ubiquitinated prior to capacitation or become ubiquitinated by the spermatozoon's intrinsic UPS during capacitation. Towards this end, we have demonstrated that boar sperm extracts are capable of substrate 
ubiquitination in a cell free system and that the sperm acrosome contains ringfinger ubiquitin ligase UBR7 (Zimmerman et al., 2014).

As aforementioned, protein phosphorylation is a key step associated with sperm capacitation. Spermatozoa undergo phosphorylation through the activation of cAMP-dependent pathways (Leclerc et al., 1996). The valosin-containing protein (VCP or p93), a sperm-borne protein dislocase capable of extracting and serving ubiquitinated membrane proteins to the $26 \mathrm{~S}$ proteasome, undergoes tyrosine phosphorylation in the presence of cAMP-elevating drugs (Geussova et al., 2002). The VCP is involved in a diverse number of cellular functions that are possibly linked to its role in membrane fusion (Rabouille et al., 1995) and proteasomal presentation of ubiquitinated proteins (Dai and $\mathrm{Li}, 2001$ ). Immunofluorescence localization in capacitated boar spermatozoa identified tyrosine phosphorylated VCP to be most concentrated in the sperm tail connecting piece, with the nonphosphorylated VCP in the distal equatorial segment, postacrosomal sheath, posterior ring, and the flagellum (Geussova et al., 2002). At this point, the physiological roles of VCP in sperm capacitation and fertilization are not known. Other UPS related proteins phosphorylated during capacitation include ubiquitinactivating enzyme E1 (UBA1) (Baker et al., 2010b), ubiquitin specific protease 38 (Arcelay et al., 2008), alpha type proteasomal subunits 6b/PSMA1-like (Arcelay et al., 2008) and alpha type 2/PSMA2 (Baker et al., 2010a), and ubiquitin itself (Platt et al., 2009). 


\section{S Core Subunit Phenotype Changes during Capacitation}

Changes in the localization and accessibility (to antibodies) of the $20 \mathrm{~S}$ core subunits occur following boar sperm capacitation; immunofluorescent labelingintensities of subunits alpha type 3 (PSMA4), beta type 6 (PSMB1) precursor, and beta 2i (PSMB10) increase (Choi et al., 2008). The increased exposure of the two beta type proteins, both of which are proteases of the $20 \mathrm{~S}$ core, might reflect their enzymatic activation and thus play a direct role in the maintenance of cellular homeostasis through proteasomal degradation of sperm proteins during capacitation. Interestingly, labeling with antibodies against the $20 \mathrm{~S}$ core as a whole show decreased fluorescence intensity after capacitation. This could be because the antibody used in the study was raised against human antigen and did not recognize porcine PSMA4 and PSMB1 subunits (Choi et al., 2008), or the proteasomes could have been lost from some spermatozoa due to premature acrosome exocytosis during capacitation.

\section{Differences in The Amounts of UPS-related Proteins in The Anterior Head Plasma Membrane}

As earlier stated, the process of capacitation is marked by an increase in fluidity of cell membranes. This allows for the relocation of proteins between the sperm head compartments. A study done by tandem mass spectrometry identified proteins of the pig sperm anterior head plasma membrane that showed an increase in abundance after capacitation (Kongmanas et al., 2015). The ubiquitinated protein content of the anterior head plasma membrane decreased by a third after 
capacitation, while there was a parallel increase in the sperm affinity for zona pellucida 3 glycoprotein (ZP3). Other UPS-related proteins that showed an increase in affinity for ZP3 included the non-ATPase 195 regulator subunits 7 (PSMD6) and 2 (PSMD1), and 20S core subunit beta type I (PSMB6) precursor, with ubiquitin domain-containing protein I showing a decreased affinity (Kongmanas et al., 2015). Noticeably, several of the proteins that changed localization or intensity, such as lactadherin MFGE8 and ACRBP were shown to co-purify with the GFP-tagged sperm proteasomes in a transgenic GFP-PSMA1 pig model (Miles et al., 2013). A related previous study of human sperm acrosome identified the $26 \mathrm{~S}$ proteasome as one of three components of a multimeric protein complex responsible for the capacitation-activated sperm-zona binding (Redgrove et al., 2011).

\section{Requirement of Ubiquitin-activating Enzyme (UBA1) for Sperm Capacitation}

The E1-type ubiquitin-activating enzyme (UBA1) is responsible for mono-ubiquitin activation prior to ubiquitin-ubiquitin and ubiquitin-substrate protein ligation (Hershko and Ciechanover, 1992). The outer acrosomal membrane remodeling of boar spermatozoa is altered under capacitating conditions with small molecule UBA1 inhibitor PYR-41. These spermatozoa remain motile but unable to fertilize in vitro (Yi et al., 2012b). Attempts to capacitate spermatozoa under such UBA1inhibiting conditions induced modifications to acrosomal proteins, acrosin-inhibitor SPINK2 and spermadhesin AQN1, that are consistent with the inhibition of their degradation/shedding (Yi et al., 2012b) normally observed during capacitation 
(Ekhlasi-Hundrieser et al., 2005). Of note, porcine sperm AQN1 coimmunoprecipitates with the 19S regulatory complex subunit PSMD8 (Yi et al., 2010a), and SPINK2 with 19S subunit PSMD4 (Yi et al., 2010b). Such interactions of the seminal-plasma derived acrosomal surface proteins with the sperm proteasome may be possible due to the translocation of the proteasome-containing zona-binding complexes evoked by aforementioned studies of human sperm capacitation (Redgrove et al., 2011). Recent observations also indicate the presence of UBA1 in human spermatozoa and an increased presence of polyubiquitinated proteins during capacitation of human spermatozoa ( $P$. Morales et al., unpublished data).

\section{Proteasomes Are Required for Acrosomal Remodeling}

Proteasomal activity appears to be necessary for acrosomal exocytosis (AE) in a number of mammalian species. Proteasomal inhibitor epoxomicin inhibited the progesterone-induced AE of capacitated bull spermatozoa (Sánchez et al., 2011). When such spermatozoa were used for in vitro fertilization, there seemed to be a time dependent effect of incubation with epoxomicin on their ability to undergo the zona oocyte induced AE in which there was a "point of no return." Specifically, 30 minutes of inhibitor incubation prior to fertilization decreased fertilization, measured by embryo cleavages by more than $60 \%$, while 60 minutes of incubation with inhibitor resulted in an $85 \%$ decrease (Sánchez et al., 2011). In humans, a pulse and chase experiment with spermatozoa bound to the human ZP revealed striking differences in the percentage of $\mathrm{AE}$ between control spermatozoa and 
those treated with lactacystin, a proteasome inhibitor. While in control spermatozoa, the percentage of $\mathrm{AE}$ increased by $265 \%$ after the chase, the percentage of $A E$ in the lactacystin-treated spermatozoa after the chase increased only by $137 \%$ (Morales et al., 2003). Similar results were found when the AE was induced with recombinant human zona proteins ZP3 and ZP4 (Chakravarty et al., 2008). Proteasome inhibitors also blocked the progesterone-induced AE, although lactacystin treatment was unable to block the ionophore-induced $A E$ (Morales et al., 2003). In addition, lactacystin interfered with the progesterone-induced $\mathrm{Ca}^{2+}$ entry into the spermatozoa. These observations suggest that proteasome's role in $A E$ is upstream of calcium influx. In the mouse model, spermatozoa treatment with proteasomal inhibitors lactacystin or epoxomicin markedly decreased in vitro fertilization rates (Pasten et al., 2005). This inhibition was due to the fact that proteasomal inhibitors reduced the zona pellucida- and the progesterone-induced AE. Of note, sperm treatment with proteasome inhibitors did not interfere with binding to intact human zonae pellucida (Wojcik et al., 2000; Morales et al., 2003) or with binding to recombinant human zona pellucida proteins (Chakravarty et al., 2008). A similar observation was made in the pig model (Sutovsky et al., 2004), where proteasome inhibitors and antibodies were used to interfere with proteasome function during IVF. These results differ from those reported by Redgrove et al. (2011) stating that sperm incubation with anti- $\alpha 3$ or anti- $\beta 1$ proteasome subunit antibodies inhibited human sperm binding to homologous intact zonae pellucidae. These differences suggest that the catalytic site of the sperm proteasome is not required for zona binding but it is needed for zona 
penetration/degradation, or that there are species differences. In support of the latter is the observation in the mouse model that proteasome inhibitors decreased sperm binding to the mouse zona pellucida (Pasten et al., 2005). It is thus possible that involvement of proteasomal proteolysis in $\mathrm{AE}$ starts with acrosomal remodeling during capacitation that primes spermatozoa for $\mathrm{AE}$, in addition to enabling acrosomal vesiculation and acrosomal shroud formation during $\mathrm{AE}$ (Gadella et al., 2008; Buffone et al., 2012). Proteasomal inhibitors also block the calcium influx needed for $\mathrm{AE}$ in marine invertebrates (Matsumura and Aketa, 1991).

\subsection{Relationship with Male Fertility}

\section{Effect of Zinc Supplementation on Male Fertility}

Decreased seminal fluid $\mathrm{Zn}^{2+}$ has been reported in cases of male infertility associated with accidental Chernobyl radiation in Ukraine (Andreychenko et al., 2016), signifying a possible relationship between $\mathrm{Zn}^{2+}$ and male fertility. Indeed, seminal fluid $\mathrm{Zn}^{2+}$ concentration is positively correlated with sperm count and normal sperm morphology (Colagar et al., 2009). Consequently, $\mathrm{Zn}^{2+}$ supplementation improves sexual dysfunction in rats (Dissanayake et al., 2009) and uremic men (Mahajan et al., 1984), likely due to the ability of $\mathrm{Zn}^{2+}$ to increase serum testosterone levels (Prasad et al., 1996). The negative effect of fatiguing bicycle exercise on thyroid hormone and testosterone levels in sedentary males is likewise prevented with $\mathrm{Zn}^{2+}$ supplementation (Kilic, 2007). Oral $\mathrm{Zn}^{2+}$ supplementation results in increased sperm counts in ram (Underwood and 
Somers, 1969) and humans (combined with inclusion of folate; review, Irani et al., 2017). Zinc supplementation also restores superoxide scavenging antioxidant capacity in asthenospermic men (Hadwan et al., 2015). Dietary $\mathrm{Zn}^{2+}$ intake and action on intraprostatic $\mathrm{Zn}^{2+}$ levels remain unknown; however, if such supplementation increases prostatic levels it could be beneficial for inhibiting HVCN1, warding off premature sperm capacitation. Goat dietary $\mathrm{Zn}^{2+}$ supplementation increases sperm plasma membrane and acrosome integrity, and percent of viable spermatozoa, also increasing seminal fluid SOD, catalase, and glutathione peroxidase activities (Narasimhaiah et al., 2018). Additionally, $\mathrm{Cu}^{2+}$ and $\mathrm{Zn}^{2+}$ dietary co-supplementation to bucks of the Osmanabadi goat breed allowed them to reached puberty 28-35 days earlier (Arangasamy et al., 2018). While $\mathrm{Zn}^{2+}$ supplementation has a positive influence on multiple male reproductive measures and can serve as male infertility therapy under certain circumstances, one study suggests that supplementation over $100 \mathrm{mg} / \mathrm{day}$ is associated with a 2.29 relative risk of advanced prostate cancer (Leitzmann et al., 2003). The exact mechanism is unclear, as $\mathrm{Zn}^{2+}$ is not generally considered a carcinogen (Plum et al., 2010); yet, increased risk is not surprising as zinc becomes cytotoxic at such concentrations and warrants dosage caution.

Few studies have assessed the addition of $\mathrm{Zn}^{2+}$ in media/semen extenders in agriculturally important livestock species propagated by artificial insemination (Al). A highly desirable property of such media is to decrease reactive oxygen species (ROS) that affect sperm function by oxidation of lipids, proteins and DNA [reviewed 
in (O'Flaherty and Fournier, 2017)]. Notably, $\mathrm{Zn}^{2+}$ supplementation would serve as an antioxidant by scavenging excessive superoxide anions (Powell, 2000), though $\mathrm{Zn}^{2+}$ homoeostasis is important, and too much $\mathrm{Zn}^{2+}$ can act as a prooxidant and cause mitochondrial oxidative stress [reviewed in (Lee, 2018a)]. Some studies have investigated $\mathrm{Zn}^{2+}$ supplementation, however they did so with the inclusion of D-aspartate and coenzyme Q10, without distinguishing which compound positively decreased lipid peroxidation and DNA fragmentation (Talevi et al., 2013) and improved embryo development (Gualtieri et al., 2014).

\section{Spermatotoxicity and Reprotoxicity of High Zinc Contamination and Zinc Deficiency}

At high soil levels, $\mathrm{Zn}^{2+}$ is reprotoxic to the terrestrial worm Enchutraeus crypticus (Posthuma et al., 1997). Few studies exist that observe $\mathrm{Zn}^{2+}$ reprotoxicity (Barceloux and Barceloux, 1999). Dietary $\mathrm{ZnCl}_{2}$ supplementation to both male and female rats at $30 \mathrm{mg} / \mathrm{kg} / \mathrm{day}$, but not 15 or $7.5 \mathrm{mg} / \mathrm{kg} / \mathrm{day}$, showed significant reduction in fertility, offspring viability, and body weight of F1 pups; however, it had no effect on litter size (male reprotoxicity alone was not observed) (Johnson et al., 2011). Nanosized ZnO toxicity induces sea urchin sperm DNA damage, but does not reduce fertility (Manzo et al., 2017). A moderate negative correlation ( $r=-0.426$ ) has been found between total flagellar $\mathrm{Zn}^{2+}$ content and percentage of morphologically normal spermatozoa in men (Henkel et al., 1999). Dietary $\mathrm{Zn}^{2+}$ deficiency studies in rat have shown reduced relative weight of the testes, epididymides, and seminal vesicles (organ by percentage of body weight) and 
reduced prostate weight (by organ weight alone). In the same study, the length of the flagellum was reduced on average by $24 \%$ in $\mathrm{Zn}^{2+}$ deficient rats, accompanied by a substantial increase in morphologically abnormal spermatozoa (especially abnormal heads, predominantly headless). Some of these defects could be due to abnormal testicular function from modulated fatty acid composition, interrupting essential fatty acid metabolism, where $\omega-6$ polyunsaturated fatty acids were highly enriched (Merrells et al., 2009). $\mathrm{Zn}^{2+}$ deficiency is known to trigger autophagy in yeast (Ding and Zhong, 2017), and elevated autophagy rate during spermatogenesis could decrease sperm count during $\mathrm{Zn}^{2+}$ deficiency. In the absence of fertilization, sperm capacitation is a terminal event leading down a rapid path of apoptosis (Jaiswal and Eisenbach, 2002) possibly from overproduction of ROS (Aitken et al., 2015) in an environment with decreased $\mathrm{Zn}^{2+}$. No studies have been performed to observe if micromolar levels of $\mathrm{Zn}^{2+}$ under capacitation-inducing conditions can prolong sperm lifespan during a fertilization competent state. If such could be achieved, fertilization would be possible with fewer spermatozoa and especially useful for artificial insemination in livestock as well as human intrauterine insemination (IUI) in place of costly IVF treatments of couples with an oligospermic male partner.

\section{Decreased Proteasomal Enzymatic Activity in Infertile Men}

Consistent with the proposed role of proteasomes in the regulation of spermiogenesis, sperm capacitation and sperm motility, asthenozoospermia and teratozoospermia in infertile men are accompanied by the reduced sperm 
proteasomal enzymatic activity, compared to fertile men (Rawe et al., 2008; Rosales et al., 2011) and down-regulation of proteasome activator subunit 4 (PSME4) gene expression in teratozoospermic men (Fu et al., 2015). Furthermore, proteasomal enzymatic activities are significantly lower in subpopulations of morphologically abnormal spermatozoa recovered from samples from otherwise healthy donors (Rosales et al., 2011). There was a positive correlation between sperm proteasomal enzymatic activity, and sperm motility and normal morphology after sperm separation by a discontinuous Percoll gradient (Rosales et al., 2011). It is generally accepted that low motility and poor sperm morphology result in decreased fertilizing ability in vivo and in vitro. It is unclear whether such spermatozoa fail to undergo capacitation and/or $\mathrm{AE}$, and if such a failure is linked to decreased proteasomal enzymatic activity. Finally, it has been shown that seminal plasma from infertile patients contains antibodies against antigens present in the sperm plasma membrane. Some of these antigens have been identified as proteasome subunits (Bohring et al., 2001). Therefore, the presence of antibodies against proteasomal subunits may be a cause of infertility.

\section{Method for Increasing Sperm Proteasome Activity, Storage Viability, and ZP Penetration}

Proteasomes depend on ATP for their structural integrity and function. Due to the proteasome exposure on the sperm surface, depletion of extracellular ATP by purified apyrase interfered with sperm proteasome function and fertilization of ascidian (Sakai et al., 2003) and porcine (Yi et al., 2009) oocytes. Conversely, 
addition of extracellular ATP stimulated sperm motility and fertilizing ability in vitro (Rodriguez-Miranda et al., 2008). The enzymatic pathway for the metabolism of inorganic pyrophosphate (PPi), which can provide an alternative ATP source under certain physiological or pathological conditions, has recently been discovered in mammalian spermatozoa (Yi et al., 2012a). The addition of PPi to boar sperm media increased the chymotrypsin-like activity of the proteasomal core (Yi et al., 2012a). Furthermore, or perhaps for that same reason, PPi enhanced boar sperm viability during storage and zona pellucida penetration during fertilization (Yi et al., 2012a). In this environment, PPi acts as a substitute for ATP; PPi is relatively more stable and less volatile than ATP, serving as a potent alternative source of energy (Chi and Kemp, 2000), which could be used as an additive to fertilization media or semen extenders for artificial insemination. Elements of the PPi-metabolizing pathway have been detected in the sperm tail connecting piece, sperm head postacrosomal sheath, equatorial segment, and overlapping the distal part of the sperm acrosome. This pathway may thus support protein phosphorylation during sperm capacitation. Since up regulation of the ATP-dependent pathways can have off target effects other than increasing proteasome activity, further research should be pursued to identify if more specific downstream substrates could increase proteasome activity without off target effects or identify if these off target effects are substantial enough that they require avoidance.

Fibronectin (FN1), a protein found in the extracellular matrix of the cumulus oophorus of the human oocyte, has been found to increase the chymotrypsin and 
trypsin-like activity of the sperm proteasome, regardless of capacitation state (Diaz et al., 2007). Additionally, FN1 increased the Tyr-phosphorylation of several proteasomal subunits. Interestingly, FN1 has recently been localized to the anterior head plasma membrane of boar spermatozoa, where it had affinity towards ZP3 glycoprotein prior to capacitation, but not after capacitation (Kongmanas et al., 2014).

\subsection{Areas for Future Exploration}

\section{Unresolved Issues}

The role of VCP in the CAMP-dependent protein tyrosine kinase pathway needs further exploration, as well as the direct and indirect activity of sperm-borne protein kinases. Further understanding of sperm proteasome subunit composition should be explored due to possibility of testis/germline specific subunit variants, as well as their role in the regulation of the events leading to capacitation. Proteomic analysis of spermatozoa from a transgenic pig with GFP tagged proteasomal subunit alpha 6 (PSMA1) identified a number of proteins that co-purified with the GFP-tagged sperm proteasomes; many of them associated with the sperm acrosome (Miles et al., 2013). A list of these proteins and proteins from additional studies that may be interactors or substrates of the sperm proteasome during capacitation is compiled in Table 1.1; their relationship with the UPS and sperm capacitation should be further explored. Identification of further proteasome substrates should also be pursued, using methods such as co- 
immunoprecipitation, protein microarrays, ubiquitome profiling using shotgun proteomics, and bioinformatics predictions.

The aforementioned, recently identified multimeric, proteasome containing protein complex expressed on the surface of human spermatozoa is tyrosine phosphorylated during capacitation. Immunofluorescence imaging localized the 205 core subunits along the flagellum and in the peri-acrosomal region of the sperm head. This complex possesses the zona adhesion affinity confirmed by the reduced binding to oocytes in the presence of antibodies against subunits of the $20 S$ proteasome (Redgrove et al., 2011). Several other papers have confirmed the sperm proteasome-zona pellucida interaction (Morales et al., 2003; Pasten et al., 2005; Yi et al., 2010a; Saldívar-Hernández et al., 2015); however, the reason for certain proteasomal subunits migrating differentially on PAGE/ Western blot before vs. after capacitation has not been investigated. The cause for this could be due to stable interactions between individual subunits and/or to their post-translational modifications (Redgrove et al., 2011). Proteasome research in other tissue types has shown that tyrosine phosphorylation of certain subunits can shift substrate specificity and such should be further explored in the proteasome of spermatozoa (Mason et al., 1996; Bose et al., 1999).

The role of the UPS in possible release of capacitated spermatozoa from the oviductal sperm reservoir should be further researched. In the cow, oviductal receptors for sperm have tentatively been identified as annexin proteins (Ignotz et 
al., 2007), however the annexin sperm receptor itself might not be responsible for release of capacitated spermatozoa. In vitro observations of a rapid loss of spermadhesin AQN1 from the boar sperm plasma membrane (Ekhlasi-Hundrieser et al., 2005) and rapid loss of the binder of sperm (BSP) proteins from capacitating bull spermatozoa (Hung and Suarez, 2012) are thought to be contributing to the release of capacitated spermatozoa from the sperm reservoir. It has been demonstrated that after the loss of BSP following capacitation, bull sperm show reduced binding to oviductal epithelium (Lefebvre and Suarez, 1996). Furthermore, BSP3 appears to be proteolytically cleaved at the $\mathrm{N}$ terminus, observed by decreased size molecular mass on western blots and confirmed by mass spectrometry. It is speculated that this proteolytic cleavage could originate from sperm surface proteases (Hung and Suarez, 2012). Beside whole protein shedding and cleavage by single molecule proteases, a role for the sperm proteasomes in the capacitation-induced release of these acrosomal surface proteins should be contemplated. A recent report indicates acrosomal AQN1 is ubiquitinated and one can speculate that this protein is degraded by proteasomes, facilitating the release of spermatozoa from the sperm reservoir upon capacitation (Yi et al., 2010a).

\section{Application and Implications}

There are many areas of application of spermatozoa UPS research. These include human infertility diagnostics detecting proteasomes and measuring proteasomal activity with specific fluorometric substrates, and the treatment for proteasome 
associated male infertility by addition of proteasome-stimulators to sperm and fertilization media. Although little is known about proteasomal proteolysis specifically at the time of sperm capacitation, a recent study shows that proteasomal proteolysis of sperm-borne ubiquitinated proteins occurs posttesticularly, during epididymal sperm maturation (Koch et al., 2015). Furthermore, multiple ubiquitin-ligases and deubiquitinases have been identified in the ejaculated mammalian spermatozoa that could support post-testicular ubiquitination and turnover of sperm proteins (Berruti 2016; Zimmerman et al., 2014). Reproductive performance of livestock species could be improved by limiting unwanted spontaneous capacitation, selecting breeding sires by their sperm proteasome activity, developing media, extenders and cryoprotectants with proteasome-stimulating components to increase conception after artificial insemination (Al) with cryopreserved semen, and improving sperm viability after semen sexing while reducing the number of spermatozoa per dose, allowing use of higher percentile indexing genetics in production systems. The discovery that inorganic pyrophosphate increases proteasomal-proteolytic and deubiquitinating activities serves as an example of semen extender supplements that can be utilized to increase sperm viability during processing, storage and postcryopreservation.

\subsection{Conclusion}

Studies reviewed here establish the role of UPS in spermatozoa capacitation as substrates and/or regulators. Further, $\mathrm{Zn}^{2+}$ plays a gatekeeping role in male 
gametes just as it does in those of the female through a variety of pathways. The 265 proteasome appears necessary for both the capacitation-induced remodeling of acrosomal surface and $A E$ itself in certain species, and the teratolasthenozoospermic human spermatozoa have decreased proteasomal enzymatic activity. Ubiquitin presence or availability in the anterior head plasma membrane decreases after capacitation and ubiquitin along with other UPS-related proteins show an increased affinity for ZP3 after this hallmark event. Prostatic seminal fluid with the highest concentration of $\mathrm{Zn}^{2+}$ found in any bodily fluids, plays a crucial role in fending off premature sperm capacitation and provides antioxidant activity, while lower concentrations of $\mathrm{Zn}^{2+}$ may be a prerequisite for successful acrosomal exocytosis.

A list of reviewed $\mathrm{Zn}$-containing and interacting proteins involved from spermatogenesis through the final preparatory steps of fertilization is summarized in Table 1.1. Further, a summary figure for $\mathrm{Zn}^{2+}$ regulation of sperm fertilization competency is supplied (Fig. 1.1) Likewise, a compilation of proteins that may interact with the sperm proteasome during capacitation/fertilization are listed in Table 1.2 and a summary of how the 26S proteasome regulates sperm capacitation (Fig. 1.2). Many mechanisms remain poorly understood and should be further explored. These include determining the relationship between the proteasome and proteins listed. Further research needs pursued to fully understand the biological role of $\mathrm{Zn}^{2+}$ in male fertility, especially to fully disclose 
the $\mathrm{Zn}$-interacting sperm proteome and its place in various cellular pathways controlling male reproductive function.

Collectively, the currently available data supports the importance of the UPS in male fertility and already hint at the same for zinc ions. These pathways could be harnessed to improve the reproductive performance of livestock and increase the success rate of human assisted reproductive therapy. Further research will advance the field of sperm and fertilization biology, provide new research tools, and ultimately optimize semen processing procedures for human infertility therapy and livestock artificial insemination.

\subsection{Acknowledgements}

Work reviewed in this chapter was supported by National Institute of Food and Agriculture (NIFA), U.S. Department of Agriculture (USDA) grant number 201167015-20025 (P.S.), 2013-67015-20961 (P.S.), 2015-67015-23231 (P.S.), USDA NIFA Graduate Fellowship award number 2017-67011-26023 (K.K.), the F.B. Miller Fellowship (K.K.), University of Missouri Graduate Studies Fellowship (K.K.), and seed funding from the Food for the 21st Century Program of the University of Missouri (P.S.). We thank Drs. Michal Zigo and Patricio Morales for their input as co-authors on these published manuscripts as well as Ms. Katherine Craighead for clerical support. PM has been supported by the Fondo Nacional de Desarrollo Científico y Tecnológico de Chile (FONDECYT) grant numbers 1080028 and 1120056. 
Flagellum Related

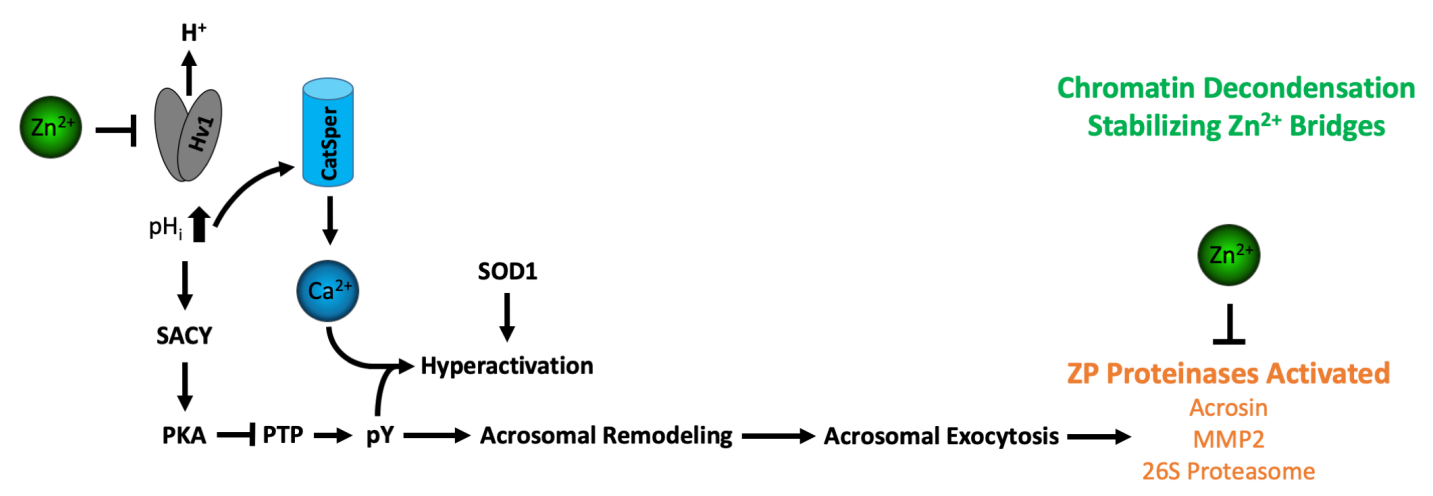

Figure 1.1. $\mathrm{Zn}^{2+}$ regulation of mammalian spermatozoa fertilization competency summary. High $\mathrm{Zn}^{2+}$ concentration $(2 \mathrm{mM})$ negatively regulates proton channel HVCN1, responsible for the rise of intracellular $\mathrm{pH}$, facilitating: 1) $\mathrm{Ca}^{2+}$ entry via CatSper and 2) protein tyrosine phosphorylation (pY), triggered by activation of soluble sperm adenylyl cyclase (SACY), increasing intracellular CAMP, activating protein kinase A (PKA) and phosphorylating protein tyrosine phosphatases (PTP) to an inactive state. Following acrosome remodeling and exocytosis, zona pellucida (ZP) proteinases (acrosin, MMP2, and the $26 \mathrm{~S}$ proteasome) implicated in endowing the spermatozoon with the ability to penetrate the $\mathrm{ZP}$ are activated. $\mathrm{Zn}^{2+}$, abundantly present in the fertilizing sperm triggered oocyte zinc spark, negatively regulates proteinase activities of spermatozoa bound to the zona or present in the perivitelline space, de-capacitating spermatozoa and serving as a newly proposed antipolyspermy defense mechanism. 


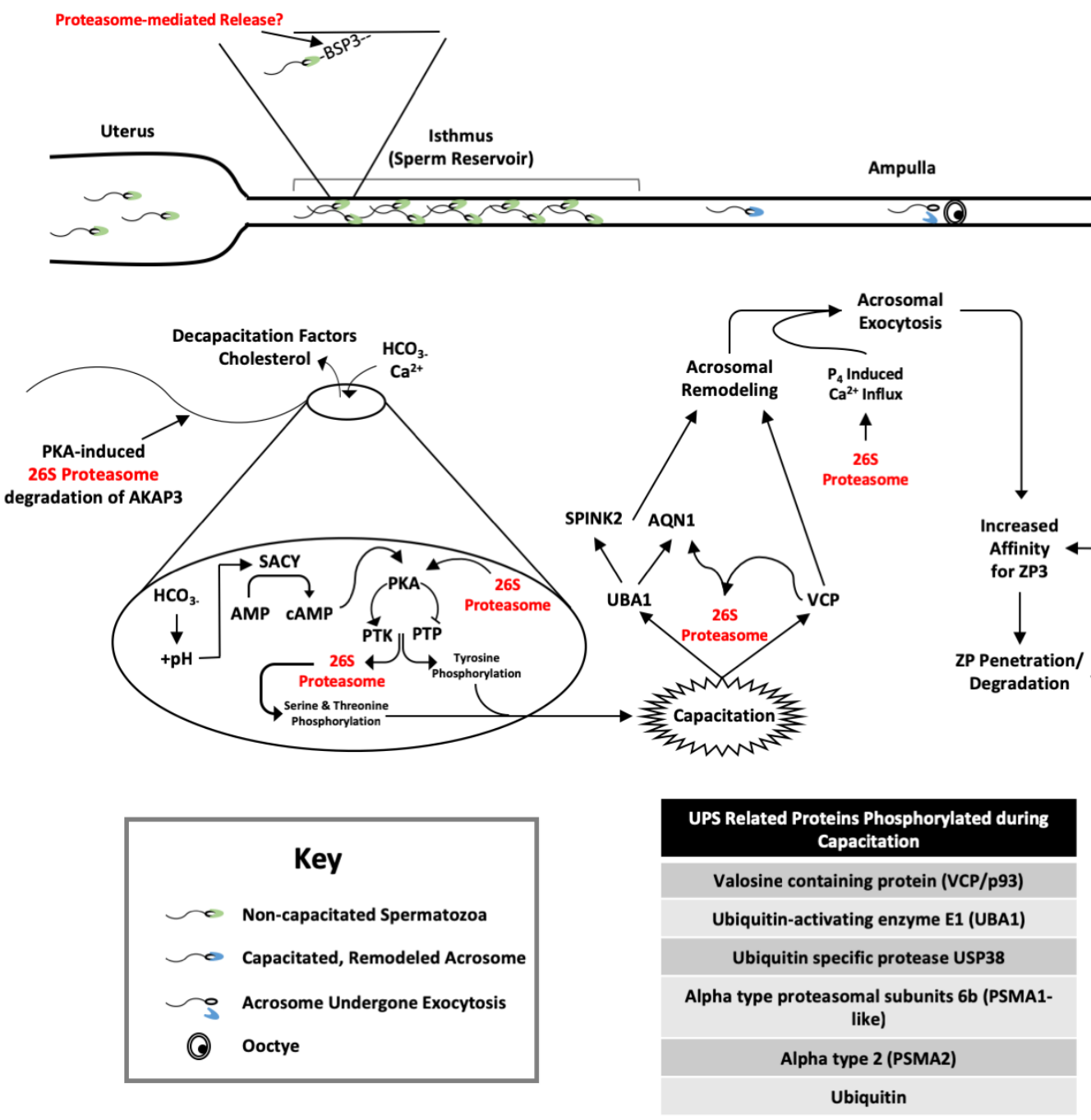

Figure 1.2. Summary of $26 \mathrm{~S}$ Proteasome-Regulated Events of Sperm Capacitation. Removal of decapacitation factors triggers a cascade of events starting with an efflux of cholesterol from the sperm plasma membrane, bicarbonate and calcium influx, increasing intracellular $\mathrm{pH}$ to activate a soluble sperm adenylyl cyclase (SACY) responsible for increasing intracellular cAMP. An increase in cAMP activates protein kinase $A(P K A)$, which phosphorylates protein tyrosine kinases (PTK) to an activated state and protein tyrosine phosphatases (PTP) to an inhibited state. Once activated by PKA, the 26S proteasome could directly or indirectly modulate substrate protein phosphorylation at serine and/or 
threonine residues. An acceleration of protein tyrosine phosphorylation occurs, that is a hallmark of sperm capacitation. During capacitation, the A-kinase anchoring protein 3 (AKAP3) is degraded by the proteasome. The E1-type ubiquitin-activating enzyme (UBA1) has been shown to play a role in modifying acrosomal proteins during capacitation, including acrosin-inhibitor serine peptidase inhibitor Kazal type 2 (SPINK2) and spermadhesin AQN1, possibly leading up to acrosomal remodeling. It is plausible to contemplate that the sperm proteasome might have a role in the capacitation-induced release of acrosomal surface proteins such as binder of sperm 3 (BSP), and in the dissociation with the oviductal epithelium sperm reservoir. Valosin-containing protein (VCP or p93) is a protein dislocase capable of extracting and serving ubiquitinated membrane proteins to the $26 \mathrm{~S}$ proteasome. The VCP has been shown to undergo tyrosine phosphorylation during capacitation. Further, preliminary data suggests VCP might have a role with events leading up to acrosomal remodeling. Previous studies suggest the proteasome is involved in events leading to acrosome exocytosis, likely upstream of progesterone-induced $\mathrm{Ca}^{2+}$ influx. Following acrosomal exocytosis, proteasomal subunits PSMD6, PSMD1, and PSMB6 show an increased affinity for zona pellucida 3 glycoprotein (ZP3). Although they may or may not to be required for sperm-zona binding depending on species, the sperm proteasomes are necessary for sperm-zona penetration. 


\begin{tabular}{|c|c|c|c|}
\hline Protein & Localization ${ }^{1}$ & Function ${ }^{2}$ & Reference \\
\hline $\begin{array}{l}\text { Metallothionein I \& } \\
\text { II }\end{array}$ & $\begin{array}{c}\text { Spermatocytes } \\
\text { (rat) }\end{array}$ & Zinc ion binding & $\begin{array}{l}\text { (Elgazar et al., } \\
2005)\end{array}$ \\
\hline Tesmin & $\begin{array}{l}\text { Spermatocytes } \\
\text { (rat) }\end{array}$ & $\begin{array}{l}\text { Developmental } \\
\text { protein }\end{array}$ & $\begin{array}{l}\text { (Elgazar et al., } \\
\text { 2005) }\end{array}$ \\
\hline $\begin{array}{c}\text { Zinc transporter } \\
\text { ZIP9 }\end{array}$ & $\begin{array}{l}\text { Spermatogenic } \\
\text { GC-2 cells (rat) }\end{array}$ & $\begin{array}{l}\text { Zinc transmembrane } \\
\text { transporter activity }\end{array}$ & $\begin{array}{c}\text { (Sugihara et al., } \\
\text { 1999) }\end{array}$ \\
\hline Protamine-2 & $\begin{array}{l}\text { Sperm nucleus } \\
\text { (man) }\end{array}$ & $\begin{array}{l}\text { Developmental } \\
\text { protein, DNA-binding }\end{array}$ & $\begin{array}{c}\text { (Barney et al., } \\
\text { 1968; Bjorndahl } \\
\text { and Kvist, 2010, } \\
\text { 2011) } \\
\end{array}$ \\
\hline Acrosin & $\begin{array}{l}\text { Acrosome } \\
\text { (man, bull) }\end{array}$ & $\begin{array}{l}\text { Hydrolase, protease, } \\
\text { serine protease }\end{array}$ & $\begin{array}{l}\text { (Johnsen et al., } \\
\text { 1982; Steven et } \\
\text { al., 1982) }\end{array}$ \\
\hline Semenoglins & $\begin{array}{l}\text { Prostatic and } \\
\text { vesicular fluids } \\
\text { (man) }\end{array}$ & - & $\begin{array}{c}\text { (Arver, 1980, } \\
\text { 1982; Arver and } \\
\text { Eliasson, 1982; } \\
\text { Siciliano et al., } \\
\text { 2000) }\end{array}$ \\
\hline ZnBP1-6 & $\begin{array}{l}\text { Seminal fluid } \\
\text { (boar, dog) }\end{array}$ & - & $\begin{array}{c}\text { (Johnson et al., } \\
1969 ; \\
\text { Mogielnicka- } \\
\text { Brzozowska et } \\
\text { al., 2011; } \\
\text { Mogielnicka- } \\
\text { Brzozowska et } \\
\text { al., 2015) } \\
\end{array}$ \\
\hline $\begin{array}{c}\text { Matrix } \\
\text { metalloproteinase- } \\
2\end{array}$ & $\begin{array}{l}\text { Seminal fluid, } \\
\text { inner } \\
\text { acrosomal } \\
\text { membrane, } \\
\text { flagellum (man, } \\
\text { bull) }\end{array}$ & $\begin{array}{l}\text { Hydrolase, } \\
\text { metalloprotease, } \\
\text { protease }\end{array}$ & $\begin{array}{c}\text { (Ferrer et al., } \\
2012 b)\end{array}$ \\
\hline $\begin{array}{c}\text { Matrix } \\
\text { metalloproteinase- } \\
9\end{array}$ & $\begin{array}{l}\text { Seminal fluid } \\
\text { and flagellum } \\
\text { (man, bull) }\end{array}$ & $\begin{array}{c}\text { Hydrolase, } \\
\text { metalloprotease, } \\
\text { protease }\end{array}$ & $\begin{array}{c}\text { (Ferrer et al., } \\
2012 \mathrm{~b})\end{array}$ \\
\hline $\begin{array}{c}\text { Superoxide } \\
\text { dismutase 1, 2, } 3\end{array}$ & $\begin{array}{l}\text { Mitochondria } \\
\text { and seminal } \\
\text { fluids (man) }\end{array}$ & $\begin{array}{l}\text { Antioxidant, } \\
\text { oxidoreductase }\end{array}$ & $\begin{array}{c}\text { (Marklund, } \\
\text { 1984; Alvarez et } \\
\text { al., 1987; Beconi } \\
\text { et al., 1993; } \\
\text { O'Flaherty et al., } \\
\text { 1997; Peeker et } \\
\text { al., 1997; Marti } \\
\text { et al., 2007; } \\
\text { Kowalowka et } \\
\text { al., 2008; }\end{array}$ \\
\hline
\end{tabular}




\begin{tabular}{|c|c|c|c|}
\hline & & & $\begin{array}{c}\text { Strzezek and } \\
\text { Fraser, 2009; } \\
\text { Park et al., } \\
\text { 2012) }\end{array}$ \\
\hline $\begin{array}{c}\text { L-lactate } \\
\text { dehydrogenase }\end{array}$ & $\begin{array}{l}\text { Seminal fluids } \\
\text { (man) }\end{array}$ & Oxidoreductase & $\begin{array}{l}\text { (Wheat and } \\
\text { Goldberg, 1983; } \\
\text { Gavella and } \\
\text { Cvitkovic, 1985; } \\
\text { Virji, 1985) }\end{array}$ \\
\hline $\begin{array}{l}\text { Putative E3 } \\
\text { ubiquitin-protein } \\
\text { ligase UBR7 }\end{array}$ & $\begin{array}{l}\text { Sperm inner } \\
\text { acrosomal } \\
\text { membrane } \\
\text { (boar) }\end{array}$ & Transferase & $\begin{array}{c}\text { (Duan and } \\
\text { Goldberg, 2003) }\end{array}$ \\
\hline $\begin{array}{c}\text { Angiotensin } \\
\text { converting enzyme }\end{array}$ & $\begin{array}{c}\text { Testis, } \\
\text { epididymis, and } \\
\text { spermatozoa } \\
\text { (man, boar, } \\
\text { stallion) }\end{array}$ & $\begin{array}{c}\text { Carboxypeptidase, } \\
\text { hydrolase, } \\
\text { metalloprotease, } \\
\text { protease }\end{array}$ & $\begin{array}{l}\text { (Zimmerman et } \\
\text { al., 2011) }\end{array}$ \\
\hline $\begin{array}{c}\text { Alkaline } \\
\text { phosphatase, } \\
\text { germ cell type }\end{array}$ & $\begin{array}{l}\text { Seminal fluid } \\
\text { and sperm } \\
\text { plasma } \\
\text { membrane } \\
\text { (man, boar) }\end{array}$ & Alkaline phosphatase & $\begin{array}{c}\text { (Bell and Lake, } \\
\text { 1962; Einarsson } \\
\text { et al., 1976; } \\
\text { Soucek and } \\
\text { Vary, 1984) }\end{array}$ \\
\hline $\begin{array}{l}\text { Putative class-II } \\
\text { fructose- } \\
\text { bisphosphate } \\
\text { aldolase } \\
\end{array}$ & $\begin{array}{l}\text { Spermatozoa } \\
\text { (bull) }\end{array}$ & Lyase & $\begin{array}{c}\text { (Gillis and } \\
\text { Tamblyn, 1984) }\end{array}$ \\
\hline $\begin{array}{c}\text { Alcohol } \\
\text { dehydrogenase } \\
\text { class-3 }\end{array}$ & $\begin{array}{l}\text { Testis, } \\
\text { spermatozoa } \\
\text { (man) }\end{array}$ & Oxidoreductase & $\begin{array}{c}\text { (Dafeldecker } \\
\text { and Vallee, } \\
\text { 1986; Khokha et } \\
\text { al., 1994) }\end{array}$ \\
\hline ADAMs & Spermatozoa & $\begin{array}{c}\text { Integrin binding, } \\
\text { metalloendopeptidase }\end{array}$ & $\begin{array}{l}\text { Review (Cho, } \\
\text { 2012) }\end{array}$ \\
\hline $\begin{array}{c}\text { Voltage-gated } \\
\text { hydrogen channel } \\
1\end{array}$ & $\begin{array}{l}\text { Flagellum } \\
\text { (man) }\end{array}$ & Ion channel & $\begin{array}{c}\text { (Lishko et al., } \\
\text { 2010) }\end{array}$ \\
\hline $\begin{array}{c}\text { G-protein coupled } \\
\text { receptor }\end{array}$ & Acrosome (bull) & $\begin{array}{l}\text { G-protein coupled } \\
\text { receptor activity }\end{array}$ & $\begin{array}{l}\text { (Michailov et al., } \\
\text { 2014) }\end{array}$ \\
\hline $\begin{array}{c}26 \mathrm{~S} \text { proteasome } \\
\text { non-ATPase } \\
\text { regulatory subunit } \\
14\end{array}$ & $\begin{array}{c}\text { Inner } \\
\text { acrosomal } \\
\text { membrane }\end{array}$ & $\begin{array}{l}\text { Hydrolase, } \\
\text { metalloprotease, } \\
\text { protease }\end{array}$ & (Yi et al., 2007) \\
\hline
\end{tabular}

${ }^{1}$ Including species reported. ${ }^{2}$ Known or predicted function. 
Table 1.2. General summary of testicular/sperm Zn-containing and interacting proteins. In order of discussion. 


\begin{tabular}{|c|c|c|c|}
\hline Protein Name & $\begin{array}{l}\text { HUGO } \\
\text { symbol }\end{array}$ & $\begin{array}{c}\text { Localization (if known in } \\
\text { fully differentiated } \\
\text { spermatozoa) }\end{array}$ & Reference \\
\hline $\begin{array}{l}\text { Valosin-containing } \\
\text { protein / p93 }\end{array}$ & VCP & $\begin{array}{l}\text { Phosphorylated: } \\
\text { connecting piece; } \\
\text { Non-phosphorylated: } \\
\text { equatorial segment, } \\
\text { postacrosomal sheath, } \\
\text { posterior ring, flagellum; } \\
\text { anterior head }\end{array}$ & $\begin{array}{l}\text { (Geussova et } \\
\text { al., 2002; } \\
\text { Ficarro et al., } \\
2003 \text { ) }\end{array}$ \\
\hline $\begin{array}{l}\text { Ubiquitin-activating } \\
\text { enzyme } E 1\end{array}$ & UBA1 & Acrosome & $\begin{array}{l}\text { (Baker et al., } \\
\text { 2010b; Yi et } \\
\text { al., 2012b) }\end{array}$ \\
\hline $\begin{array}{l}\text { Ubiquitin specific } \\
\text { protease } 38\end{array}$ & USP38 & $\begin{array}{l}\text { (localization not } \\
\text { determined) }\end{array}$ & $\begin{array}{l}\text { (Baker et al., } \\
2010 \mathrm{~b})\end{array}$ \\
\hline $\begin{array}{l}\text { Proteasomal Subunit } \\
\text { Alpha Type } 6 \mathrm{~b}\end{array}$ & PSMA1 & Acrosome & $\begin{array}{c}\text { (Arcelay et al., } \\
\text { 2008) }\end{array}$ \\
\hline Fibronectin & FN & $\begin{array}{l}\text { Anterior head plasma } \\
\text { membrane }\end{array}$ & $\begin{array}{l}\text { (Kongmanas } \\
\text { et al., 2014) }\end{array}$ \\
\hline Spermadhesin & AQN1 & Acrosome & $\begin{array}{l}\text { (Jonakova et } \\
\text { al., 1998; Yi et } \\
\text { al., 2010a; Yi } \\
\text { et al., 2012b) }\end{array}$ \\
\hline Spermadhesin & AWN & Acrosome & $\begin{array}{l}\text { (Jonakova et } \\
\text { al., 1998; } \\
\text { Miles et al., } \\
\text { 2013) }\end{array}$ \\
\hline $\begin{array}{c}\text { Major seminal plasma } \\
\text { glycoprotein PSP-I } \\
\text { precursor }\end{array}$ & N/A & $\begin{array}{c}\text { Anterior head plasma } \\
\text { membrane }\end{array}$ & $\begin{array}{l}\text { (Miles et al., } \\
2013 ; \\
\text { Kongmanas et } \\
\text { al., 2014) }\end{array}$ \\
\hline $\begin{array}{c}\text { Seminal plasma sperm } \\
\text { motility inhibitor A1 }\end{array}$ & AQN3 & $\begin{array}{l}\text { (localization not } \\
\text { determined) }\end{array}$ & $\begin{array}{l}\text { (Miles et al., } \\
\text { 2013) }\end{array}$ \\
\hline $\begin{array}{c}\text { Seminal Plasma } \\
\text { Protein pB1 precursor }\end{array}$ & N/A & $\begin{array}{l}\text { (localization not } \\
\text { determined) }\end{array}$ & $\begin{array}{l}\text { (Miles et al., } \\
\text { 2013) }\end{array}$ \\
\hline $\begin{array}{c}\text { Acrosin-binding protein } \\
\text { (degradation product) }\end{array}$ & ACRBP & $\begin{array}{l}\text { (localization not } \\
\text { determined) }\end{array}$ & $\begin{array}{l}\text { (Miles et al., } \\
\text { 2013) }\end{array}$ \\
\hline $\begin{array}{c}\text { Seminal Plasma } \\
\text { Acrosin Inhibitor A1 }\end{array}$ & SPINK2 & $\begin{array}{l}\text { Anterior head plasma } \\
\text { membrane }\end{array}$ & $\begin{array}{l}\text { (Miles et al., } \\
2013 ; \\
\text { Kongmanas et } \\
\text { al., 2014) }\end{array}$ \\
\hline $\begin{array}{l}\text { A-disintegrin-and- } \\
\text { metalloproteinase } \\
\text { domain } 5 \text { (ADAM5) }\end{array}$ & ADAM5 & $\begin{array}{l}\text { Postacrosomal sheath } \\
\text { (unpublished) }\end{array}$ & $\begin{array}{l}\text { (Miles et al., } \\
2013 \text { ) }\end{array}$ \\
\hline
\end{tabular}




\begin{tabular}{|c|c|c|c|}
\hline $\begin{array}{l}\text { A-disintegrin-and- } \\
\text { metalloproteinase } \\
\text { domain 20-like } \\
\text { (ADAM20-like) }\end{array}$ & $\begin{array}{l}\text { ADAM20- } \\
\text { like }\end{array}$ & $\begin{array}{c}\text { Anterior head plasma } \\
\text { membrane }\end{array}$ & $\begin{array}{l}\text { (Mori et al., } \\
\text { 2012; Miles et } \\
\text { al., 2013) }\end{array}$ \\
\hline Lactadherin & MFGE8 & Acrosome & $\begin{array}{c}\text { (Ensslin et al., } \\
\text { 1998; } \\
\text { Petrunkina et } \\
\text { al., 2003; } \\
\text { Miles et al., } \\
\text { 2013) }\end{array}$ \\
\hline Ropporin 1-like & ROPN1L & $\begin{array}{l}\text { (localization not } \\
\text { determined) }\end{array}$ & $\begin{array}{l}\text { (Miles et al., } \\
\text { 2013) }\end{array}$ \\
\hline Ubiquitin ligase UBR7 & UBR7 & Acrosome & $\begin{array}{l}\text { (Zimmerman } \\
\text { et al., 2014) }\end{array}$ \\
\hline Ubiquitin-C isoform X1 & UBC & Acrosome & $\begin{array}{l}\text { (Labas et al., } \\
\text { 2015) }\end{array}$ \\
\hline Ubiquitin & UBB & $\begin{array}{l}\text { Acrosome/anterior head } \\
\text { plasma membrane }\end{array}$ & $\begin{array}{l}\text { (Kongmanas } \\
\text { et al., 2014) }\end{array}$ \\
\hline $\begin{array}{l}\text { Ubiquitin domain- } \\
\text { containing protein } 1\end{array}$ & UBTD1 & $\begin{array}{l}\text { Anterior head plasma } \\
\text { membrane }\end{array}$ & $\begin{array}{l}\text { (Kongmanas } \\
\text { et al., 2014) }\end{array}$ \\
\hline $\begin{array}{l}\text { 26S proteasome } \\
\text { regulatory subunit } 7\end{array}$ & PSMD7 & $\begin{array}{l}\text { Anterior head plasma } \\
\text { membrane }\end{array}$ & $\begin{array}{l}\text { (Kongmanas } \\
\text { et al., 2014) }\end{array}$ \\
\hline $\begin{array}{l}\text { Zona pellucida } 3 \\
\text { glycoprotein (ZP3) }\end{array}$ & ZPC & $\begin{array}{l}\text { Zona pellucida protein; } \\
\text { binds to anterior head } \\
\text { plasma membrane }\end{array}$ & $\begin{array}{l}\text { (Kongmanas } \\
\text { et al., 2014) }\end{array}$ \\
\hline $\begin{array}{c}\text { A Kinase (PRKA) } \\
\text { Anchor Protein } 3 \\
\text { (AKAP3) }\end{array}$ & AKAP3 & Sperm tail principal piece & $\begin{array}{l}\text { (Hillman et al., } \\
\text { 2013) }\end{array}$ \\
\hline Protein kinase $\mathrm{A}$ & PKA & $\begin{array}{l}\text { Principal piece and } \\
\text { acrosome }\end{array}$ & $\begin{array}{l}\text { (Morales et } \\
\text { al., 2007) }\end{array}$ \\
\hline calcineurin/PP2B & PPP2CB & $\begin{array}{l}\text { Along the flagellum; base } \\
\text { of the head }\end{array}$ & $\begin{array}{l}\text { Unpublished } \\
\text { (P. Morales) }\end{array}$ \\
\hline $\begin{array}{c}\text { Protein phosphatase } 1 \\
\gamma\end{array}$ & PPP1CC & $\begin{array}{c}\text { post-acrosomal, and } \\
\text { partially connecting, middle } \\
\text { and principal piece of the } \\
\text { flagellum }\end{array}$ & $\begin{array}{l}\text { Unpublished } \\
\text { (P. Morales) }\end{array}$ \\
\hline
\end{tabular}

Table 1.1. Compilation of proteins that may interact with the sperm proteasome during capacitation/fertilization. N/A=not annotated in HUGO gene list. 


\section{CHAPTER 2}

\section{PART 1: CHARACTERIZING ZINC ION DISTRUBUTION IN MAMMALIAN SPERMATOZOA}

Components published as part of:

Kerns K, Zigo M, Drobnis EZ, Sutovsky M, Sutovsky P. 2018. Zinc lon Flux during Mammalian Sperm Capacitation. Nature Communications 9, Article number: 2061. DOI:10.1038/s41467-018-04523-y

\subsection{Abstract}

For decades, the role of the divalent ion calcium was the focus of understanding mechanisms leading to sperm fertilization competency and management of semen storage. Little focus has been placed on other divalent ions, including zinc ion $\left(\mathrm{Zn}^{2+}\right)$. Here, we document a novel biological phenomenon of a unique $\mathrm{Zn}^{2+}$ distribution (further zinc signature) associated with mammalian spermatozoa from the round spermatid stage of spermiogenesis, to epididymal maturation, ejaculation, and up to 72 hours of liquid semen storage. Using image-based flow cytometry (IBFC), we identified four distinct zinc signatures. These findings represent a fundamental shift in the understanding of mammalian fertilization, paving the way for improved semen analysis, in vitro fertilization (IVF) and artificial insemination (Al). 


\subsection{Introduction}

lons play an important role in sperm function (Shukla et al., 2012). In the last few decades, most of the effort has been placed on the study of calcium $\left(\mathrm{Ca}^{2+}\right)$ due to its role in sperm capacitation and acrosomal exocytosis (Yanagimachi and Usui, 1974; Yanagimachi, 1982). While $\mathrm{Ca}^{2+}$ plays a major role in late sperm capacitation events and may be involved in producing a subpopulation of fertilization competent spermatozoa (Luque et al., 2018), the role of zinc ions $\left(\mathrm{Zn}^{2+}\right)$ in spermatozoa has been overlooked. While this could be due to previously limited $\mathrm{Zn}^{2+}$ detection methods, it may have been because early sperm function studies were based mostly on epididymal spermatozoa from lower order mammals, rodents. Spermatozoa in those studies were not representative of physiologically normal spermatozoa that come into contact with seminal plasma, known for having the highest concentration of zinc found in all bodily fluids (Liu et al., 2009).

Structurally, zinc is believed to form Zn-bridges, regulating disulfide cross-links of the DNA packaging protamines in the sperm nucleus. This results in condensed chromatin, protecting DNA from damage on their path to fertilization (Kvist, 1980a; Bjorndahl and Kvist, 2014). Further, zinc trafficking proteins, both zinc transport proteins (ZnTs) and Zrt, Irt-like proteins (ZIPs) have been found in spermatozoa throughout the testis and epididymis (Foresta et al., 2014). The localization of these zinc trafficking proteins suggests that $\mathrm{Zn}^{2+}$ has an important role in

spermatozoa development and fertility; however, to date $\mathrm{Zn}^{2+}$ localization has not been well characterized in mammalian spermatozoa. Here, we document four 
distinct types of sperm zinc distribution patterns (further zinc signatures) present in domestic boar, bull, and human spermatozoa and removed by zinc chelation. This all together supports a new biological understanding of $\mathrm{Zn}^{2+}$ in sperm function.

(A comprehensive Materials and Methods is located on page 111.)

\subsection{Results}

\section{Zinc content of the developing and maturing spermatozoa}

We used epifluorescence microscopy to trace the sperm zinc signature using $\mathrm{Zn}$ probe FluoZin ${ }^{\mathrm{TM}}$-3 AM (FZ3), DNA stain Hoechst 33342, and plasma membraneintegrity reflecting DNA stain propidium iodide $(\mathrm{PI})$, which is taken up by exclusively by cells with a compromised/remodeled plasma membrane. Relatively little zinc was localized to the developing round spermatids (as reported by lack of FZ3 fluorescence; Fig. 2.1a). Fully differentiated boar spermatozoa had increased FZ3 fluorescence as compared to the round spermatids, localized to the sperm head and midpiece (Fig. 2.1b). This pattern and relative abundance of $\mathrm{Zn}$-induced fluorescence was similar in epididymal spermatozoa (Fig. 2.1c); however, as spermatozoa progressed from the caput to the cauda epididymis, there was a statistical trend of the median FZ3 fluorescence area to increase $(93.0 \pm 2.8$ versus $137.5 \pm 21.9$ standard FlowSight area units; presented \pm standard deviation; $P$ value $=0.08$; detected by image-based flow cytometry). As shown in Fig. 2.1e, this 
is reflective of the of the Zn-rich cytoplasmic droplet's progression down the sperm tail midpiece.

\section{Ejaculated mammalian spermatozoa possess four distinct zinc signatures}

We identified four distinct types of sperm zinc signatures conserved across boar (Fig. 2.2a-e), bull (Fig. 2.2f-i), and human spermatozoa (Fig. 2.2j-m): high $\mathrm{Zn}^{2+}$ presence in the sperm head and whole sperm tail (signature 1; Fig. 2.2a, f, j), medium-level (based on relative intensity of fluorescence in FlowSight measurements) $\mathrm{Zn}^{2+}$ presence in both the sperm head and sperm tail midpiece (signature 2, spermatozoa undergoing early stage capacitation; Fig. 2.2b, g, k), $\mathrm{Zn}^{2+}$ presence in the midpiece only (signature 3/capacitated state signature in spermatozoa that underwent capacitation and may be dying; Fig. 2.2c, h, I), and no $\mathrm{Zn}^{2+}$ presence (signature 4, spermatozoa with compromised/remodeled plasma membrane; Fig. 2.2d, i, m). Spermatozoa after 72 hours of storage in Beltsville Thaw Solution (BTS semen extender) show varied zinc signatures (Fig. 2.2e).

Next, we wanted to analyze the zinc signature using super resolution confocal microscopy. Originally, this was intended for simultaneously capturing FZ3 and brightfield (transmitted light) videos to document the previously unknown posteriorto-anterior boar sperm head membrane modification; however, documenting such phenomena was unsuccessful due to technical limitations. Imaging with super resolution microscopy did however yield identification of a distinct FZ3 fluorescent localization patterns at the junction of the equatorial segment and acrosome (Fig. 
2.3a and d). FZ3 fluorescence in the sperm head disappeared upon plasma membrane and acrosomal modification. This is shown by the incorporation of propidium iodide ( $\mathrm{PI}$; red, membrane permeability probe) and lectin PNA (Arachis hypogea/peanut agglutinin; cyan, acrosomal status probe) conjugated to Alexa Fluor ${ }^{\text {TM }} 647$ (PNA-AF647) in Fig. 2.3d. The relationship this might have with sperm capacitation is further investigated in Chapter 3.

While unable to capture video footage in boar, we were able to acquire still images of the sperm head membrane change progression in bull spermatozoa. This is illustrated in Fig. 2.4, occurring during the transition from zinc signature 2 to zinc signature 3. In Fig. 2.4a, the head of a signature 2 spermatozoon is displayed. Upon early plasma membrane modification (Fig. 2.4b), PI can be seen infiltrating the posterior aspect of the sperm head while a relative decrease in FZ3 fluorescence is observed in the postacrosomal sheath. As more PI is incorporated into the sperm head, zinc is reported in the bull sperm acrosome (Fig. 2.4c) until finally, full PI incorporation into the sperm nucleus and no acrosomal localized FZ3 is observed (Fig. 2.4d).

Cell-permeant $\quad \mathrm{Zn}^{2+} \quad$ chelator $\quad \mathrm{N}, \mathrm{N}, \mathrm{N}^{\prime}, \mathrm{N}^{\prime}-$ Tetrakis(2-pyridinylmethyl)-1,2ethanediamine (TPEN) removed a majority of FZ3 fluorescence to signature 3 and 4 states (Fig. 2.5a) compared to TPEN vehicle (Fig. 2.5b) but TPEN did not reduce Fluo-4 calcium probe intensity compared to vehicle (Fig. 2.5c). Remaining FZ3 fluorescence is likely due to $\mathrm{Zn}^{2+}$ tightly bound within the mitochondrial sheath, as 
$\mathrm{Zn}^{2+}$ has been previously detected there by electron microscopy (Stoltenberg et al., 1997).

\subsection{Discussion}

Fully differentiated testicular (Fig. 2.1b) and epididymal spermatozoa (Fig. 2.1c) have less relative $\mathrm{Zn}^{2+}$ than ejaculated spermatozoa (Fig. 2.2a-d; based on relative $Z n$-probe fluorescence). Though there was only a trend in the median FZ3 area increase during epididymal maturation, the biological replicate number was limited to two. Epididymal maturation between these two replicates may have been at different stages since the migration of the Zn-rich cytoplasmic droplets did not progress in the second replicate as much as in the first one (153 vs 122 median standard FlowSight area units). Results shown here are consistent with Foresta et al., (2014), who previously reported increased Zn-probe fluorescence (using Santa Cruz ZnAF-2DA; similarly, a cell permeable probe) progressing through testicular, epididymal, and ejaculated spermatozoa.

The increased $\mathrm{Zn}^{2+}$ uptake occurring from the round spermatid to fully differentiated spermatozoa is likely mediated by testicular zinc transporters described previously (Foresta et al., 2014). The increased $\mathrm{Zn}^{2+}$ content of ejaculated spermatozoa in comparison to epididymal spermatozoa (as detected by relative FZ3 fluorescence) is likely due to a contact with Zn-rich seminal plasma upon ejaculation. The accessory sex glands may provide differing sperm zinc signature subpopulations depending on which spermatozoa are surrounded by the 
pulsatile differences in specific accessory sex gland fluids that occurs throughout boar ejaculation.

It is unclear if the posterior-to-anterior sperm head PI changes observed are indicative of an entire membrane change in a posterior-to-anterior directed wave, or if this is indicative of simply PI crossing the modified (i.e. more fluid and permeable) sperm plasma membrane and nuclear envelope in the post-acrosomal region. However, since $\mathrm{Zn}^{2+}$ removal mimics this pattern, it is likely the former. The FZ3 fluorescence localized to the bull acrosome likely represents $\mathrm{Zn}^{2+}$ attached to or dissociated from acrosomal Zn-sensing receptor (ZnR) GPR39 of the G-proteincoupled receptor (GPCR). This $\mathrm{ZnR}$ has been reported previously in bull spermatozoa and is believed to regulate acrosomal exocytosis (Michailov et al., 2014).

In fresh boar spermatozoa, the posterior-to-anterior sperm head membrane modification illustrated in bull (Fig. 2.4) occurs in the matter of 2-3 seconds and thus was impossible to acquire with available equipment. Still image acquisition might have been possible in bull spermatozoa due to the use of cryopreserved spermatozoa. It is known that cryopreservation inflicts freezing-induced, capacitation-like changes on spermatozoa. It is unclear if these changes occurred slower in bull compared to boar because of freezing-induced damage or if it may be due to species differences in sperm plasma membrane composition, such as different content of cholesterol (presumably higher in bull). If this is a side effect of 
cryopreservation, then cryopreservation could affect sperm Zn-signaling modifications and sperm fertility. Routine assessment of the zinc signature could prove useful in future attempts to improve cryopreservation methods. The specific relationship between the sperm zinc signature and sperm capacitation will be analyzed and discussed in detail in Chapter 3 , including possible reasons for all four sperm zinc signatures being present after 72 hours of liquid preserved boar semen (Fig. 2.2e; Chapter 4).

In conclusion, we have documented four unique zinc signatures associated with mammalian spermatozoa and sperm $\mathrm{Zn}^{2+}$ uptake from the round spermatid through ejaculation. The Zn-chelator TPEN quenches the sperm zinc signature. The signature 2 to signature $3 \mathrm{Zn}$-efflux appears to coincide with a posterior-toanterior plasma membrane modification. These findings present a new possible biomarker for elucidating sperm fertilization competency and understanding of mammalian fertilization. 


\subsection{Acknowledgements}

Supported by the National Institute of Food and Agriculture (NIFA), U.S. Department of Agriculture (USDA) grant number 2015-67015-23231 (PS), USDA NIFA Graduate Fellowship award number 2017-67011-26023 (KK), grant number 5 R01 HD084353-02 from NIH National Institute of Child and Human Development (PS), and seed funding from the Food for the $21^{\text {st }}$ Century Program of the University of Missouri (PS). We thank staff of the National Swine Research and Resource Center, University of Missouri, as well as Dr. Randall Prather and his associates for wild-type boar semen collection, funded by National Institutes of Health (NIH) U42 OD011140. 

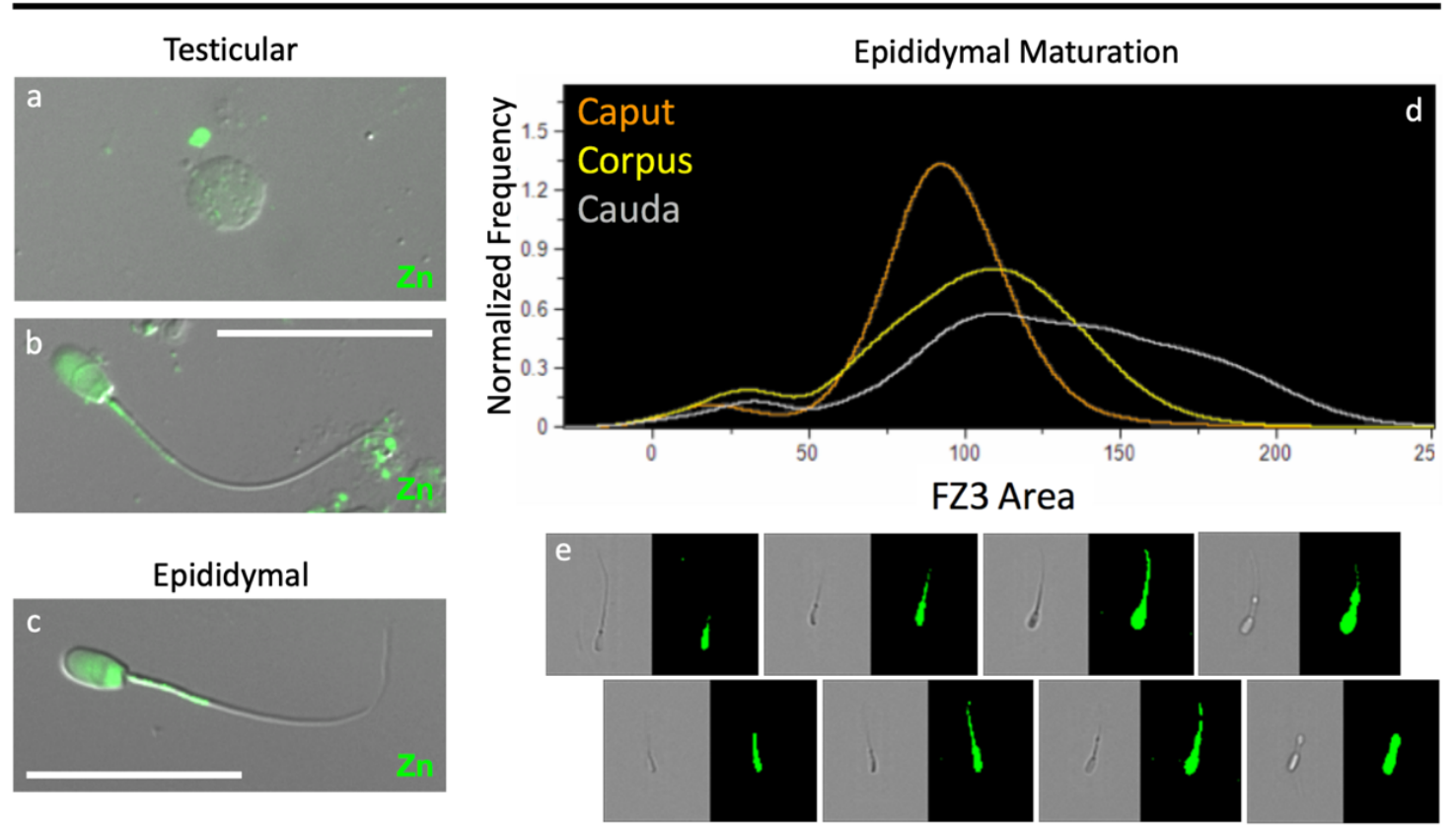

Figure 2.1. Zinc content of the developing boar spermatozoa prior to ejaculation.

a, Epifluorescence microscopy of a boar round spermatid lack Zn-probe FZ3 fluorescence (green). b, Fully differentiated boar spermatozoa have low relative $\mathrm{Zn}$-induced fluorescence in the sperm head and midpiece (as compared to ejaculated spermatozoa shown in Fig. 2.2) including in epididymal spermatozoa. d, Image-based flow cytometer histogram illustrating increase in overall FZ3 fluorescence area during sperm epididymal maturation from the caput (orange) through the cauda (gray) with typical image gallery examples (e) of brightfield and FZ3 channel. Representative of 2 biological replicates. 


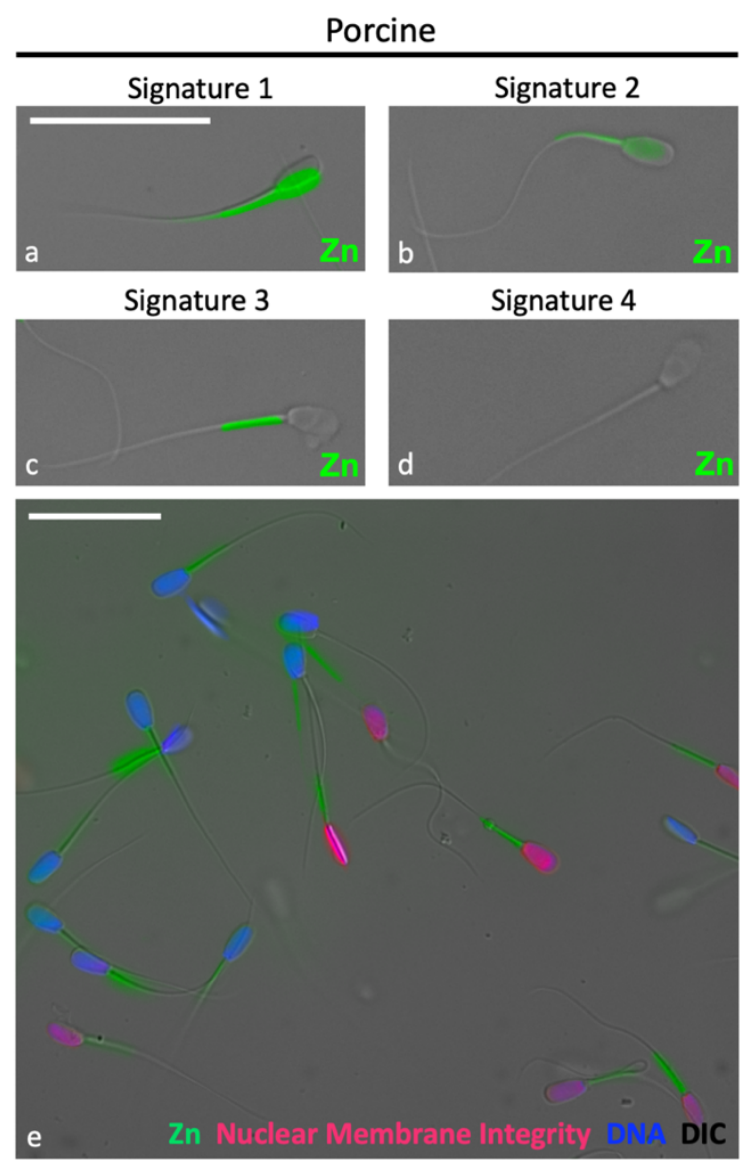

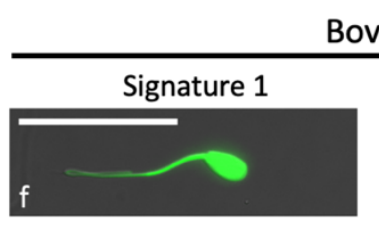

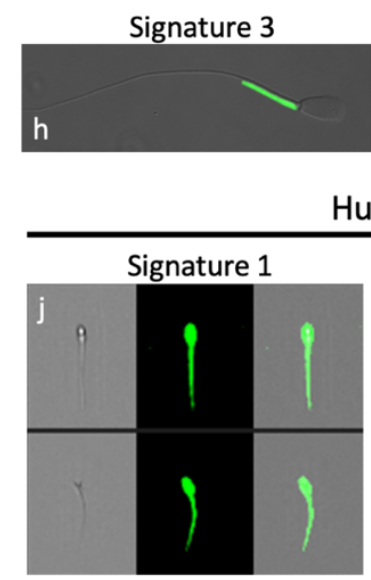

Signature 3

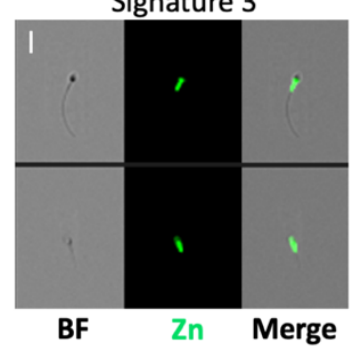

Signature 2

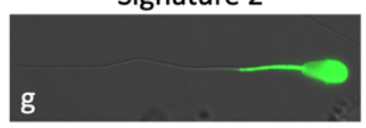

Signature 4

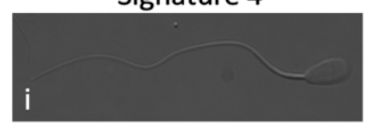

Human

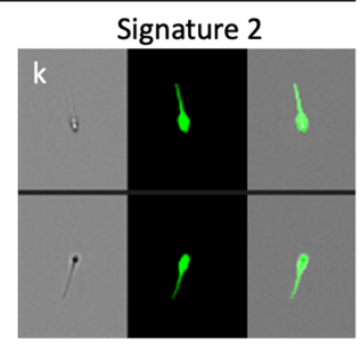

Signature 4

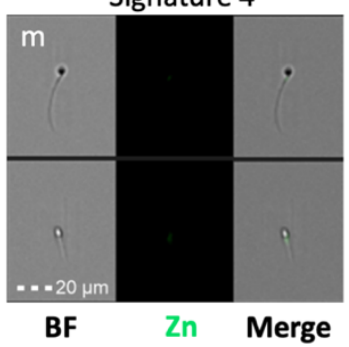

Figure 2.2. Mammalian sperm zinc signature. a-d, Epifluorescence microscopy of boar sperm zinc signature (green). e, Boar spermatozoa after 72 hours of storage in Beltsville Thaw Solution (BTS semen extender) show varied zinc signatures with compromised nuclear membrane integrity-reflecting propidium iodide (red) and nucleus (blue, Hoechst 33342) staining. Imprecise fluorescent to brightfield overlay illustrates motile status. f-i, Epifluorescence microscopy of bull sperm zinc signatures (scale bars: $25 \mu \mathrm{m}$ ). j-m, FlowSight image gallery of human sperm zinc signatures (scale bar: $20 \mu \mathrm{m}$ ). 


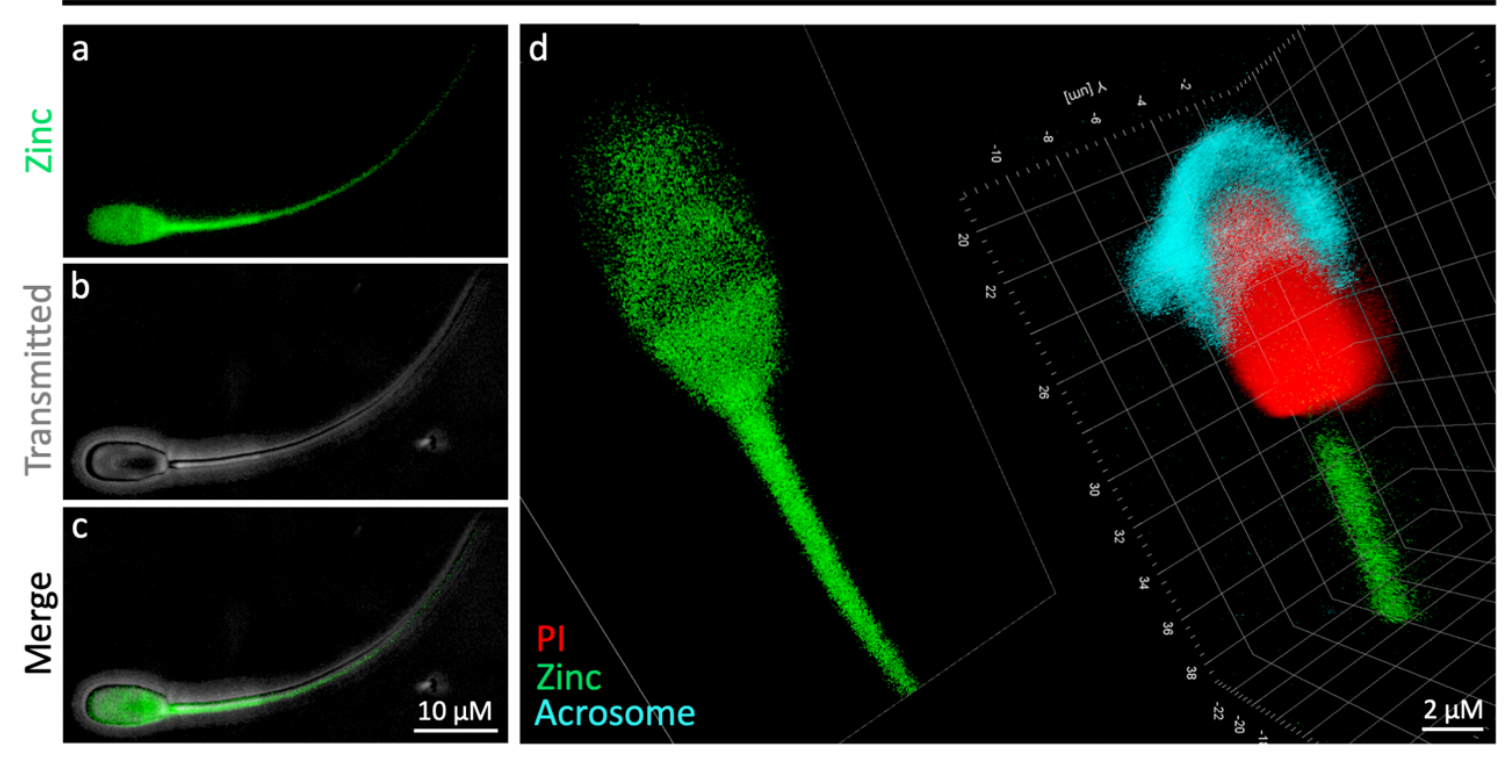

Figure 2.3. Super resolution confocal microscopy of boar sperm zinc signature. a, Zn probe FZ3 fluorescence (green) with transmitted light (b) and merged (c; scale bar: $10 \mu \mathrm{m})$. d, Boar sperm head displaying capacitation-induced zinc signature changes with modified acrosome (cyan) and nuclear membrane (red; propidium iodide, Pl; scale bar: $2 \mu \mathrm{m})$. 


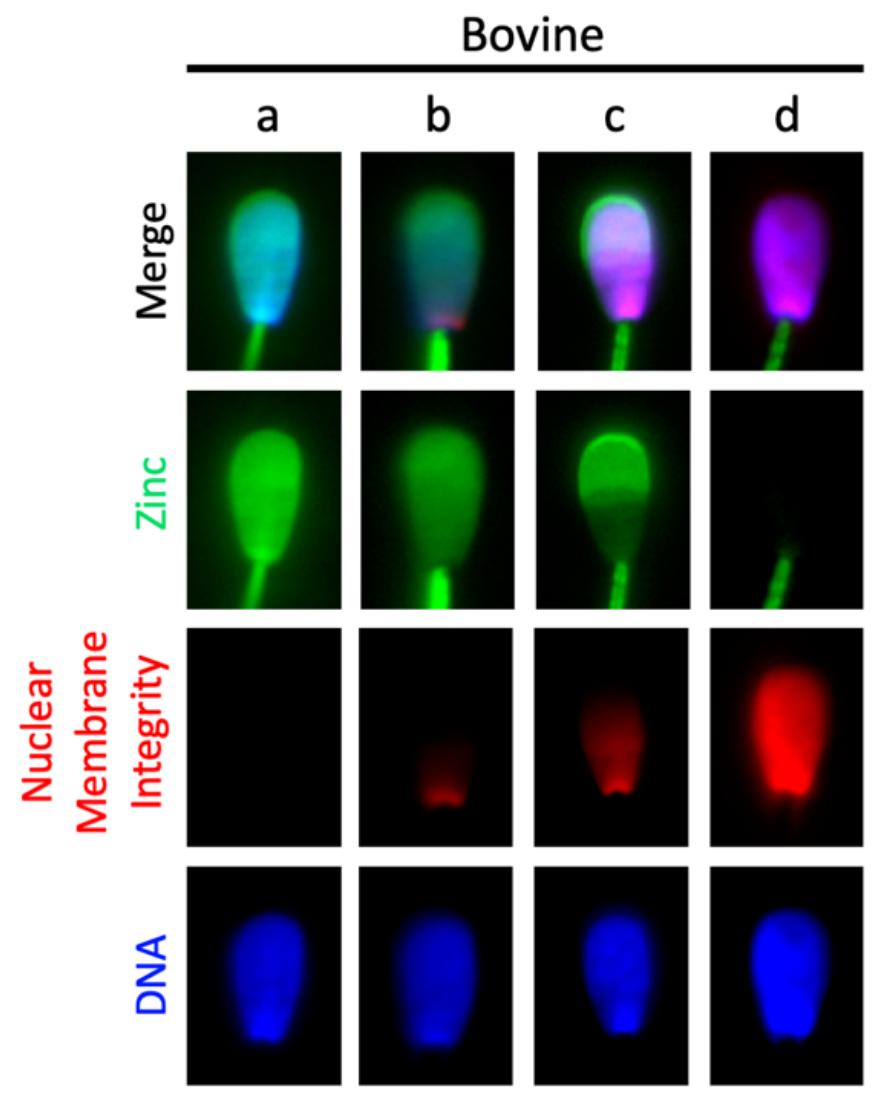

Figure 2.4. Bull sperm head $\mathrm{Zn}$-efflux, reflected by transition from signature 2 to 3 , is associated with a posterior-to-anterior nuclear membrane modification wave. a, Epifluorescence microscopy of entire sperm-head $\mathrm{Zn}$ localization at zinc signature 2 (green). b, Nuclear membrane modification-reflecting probe propidium iodide (red) showing initial plasma membrane modification originating at the posterior sperm head and lesser Zn-probe fluorescence intensity. c, Increased nuclear membrane modification enabled the exodus of $\mathrm{Zn}$-probe fluorescence from postacrosomal sheath with remaining fluorescence restricted to the acrosome. d, Full acquisition of sperm zinc signature 3 state, with propidium iodide localized to the entire sperm nucleus and removal of acrosomal Zn (detectable by lack of FZ3 fluorescence). 

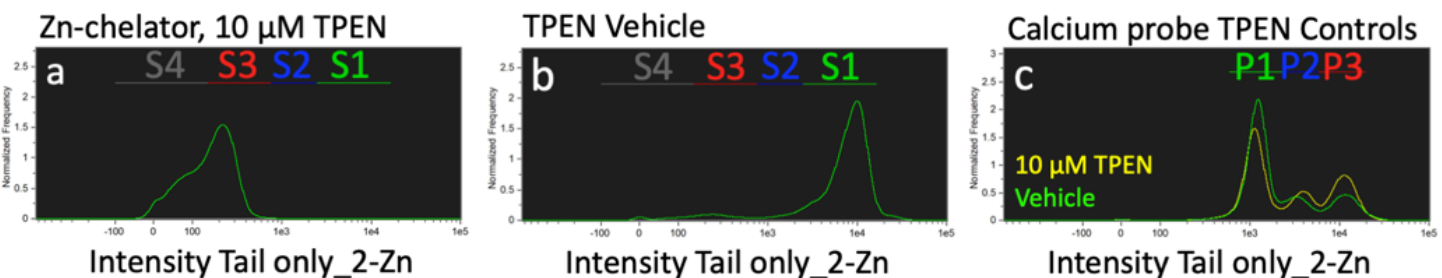

Figure 2.5. FZ3 zinc probe validation controls. a, $10 \mu \mathrm{M} Z \mathrm{Zn}$-chelator TPEN altered the zinc signature compared to $\mathbf{b}$, TPEN vehicle treatment. c, Fluo4 calcium probe TPEN and vehicle control to show no decrease in TPEN-treated calcium levels. 


\section{CHAPTER 3}

\section{PART 2: ZINC ION FLUX DURING MAMMALIAN SPERM CAPACITATION}

Components published as part of:

Kerns K, Zigo M, Drobnis EZ, Sutovsky M, Sutovsky P. 2018. Zinc lon Flux during Mammalian Sperm Capacitation. Nature Communications 9, Article number: 2061. DOI:10.1038/s41467-018-04523-y

Published as cover diagram for (Figure 3.7b):

Systems Biology in Reproductive Medicine 2019, 65. Link.

\subsection{Abstract}

Sperm capacitation, the ultimate maturation event preparing mammalian spermatozoa for fertilization, was first described in 1951, yet its regulatory mechanisms remain poorly understood. The capacitation process encompasses an influx of bicarbonate and calcium ions, removal of decapacitating factors, changes of $\mathrm{pH}$ and sperm proteasomal activities, and the increased protein tyrosine phosphorylation. Here, we document a novel biological phenomenon of a unique zinc $\left(\mathrm{Zn}^{2+}\right)$ ion redistribution associated with mammalian sperm in vitro capacitation (IVC). Using image-based flow cytometry (IBFC), we identified four distinct types of sperm zinc ion distribution patterns (further zinc signature) and their changes during IVC. The zinc signature was altered after sperm capacitation, reduced by proteasomal inhibitors, and maintained with addition of external $\mathrm{ZnCl}_{2}$. 
These findings represent a fundamental shift in the understanding of mammalian fertilization, paving the way for improved semen analysis, in vitro fertilization (IVF) and artificial insemination (Al).

\subsection{Introduction}

A zinc spark (an exocytotic event releasing billions of zinc ions) issued from the oocyte induced by the spermatozoa at fertilization (Que et al., 2015) is implicated as a novel biomarker of mammalian embryo quality and developmental potential (Zhang et al., 2016). To date, zinc ion $\left(\mathrm{Zn}^{2+}\right)$ fluxes have not been well characterized in mammalian spermatozoa, though sperm-flagellar voltage-gated proton channel HVCN1, negatively regulated by $\mathrm{Zn}^{2+}$, has been implicated as the main proton extrusion mechanism during mammalian sperm capacitation (Lishko et al., 2010). This channel regulates intracellular $\mathrm{pH}$ and consequently, is thought to be responsible for $\mathrm{Ca}^{2+}$ entry via opening of the CatSper channel, all of these events coinciding with PKA activation and the hallmark increase of protein tyrosine phosphorylation during boar sperm capacitation (Tardif et al., 2003; Boerke et al., 2008). Further, the $26 S$ proteasome, a multi-subunit ubiquitin-dependent protease, regulates fertilization at multiple steps from spermiogenesis to sperm penetration of the oocyte zona pellucida (Sutovsky, 2011) including certain aspects of sperm capacitation (Kerns et al., 2016). In particular, the A-kinase anchoring protein AKAP3 is degraded by the ubiquitin-proteasome system (UPS) during bull sperm capacitation (Hillman et al., 2013), and the E1-type ubiquitin-activating enzyme (UBA1) inhibitor PYR-41 alters acrosomal remodeling. Additionally proteasomal 
inhibitors hinder the capacitation-associated shedding of acrosin-inhibitor serine peptidase inhibitor Kazal type 2 (SPINK2) and spermadhesin AQN1 (Yi et al., $2012 b)$ in the boar.

Here, we use image-based flow cytometry (IBFC) to document four distinct types of sperm zinc signature and their changes during in vitro capacitation (IVC) of domestic boar, bull and human spermatozoa, altered after sperm capacitation, further reduced under proteasome inhibiting IVC conditions, and maintained with addition of external $\mathrm{ZnCl}_{2}$. The zinc shield established by the oocyte following fertilization could derail such sperm zinc signaling as an added barrier to pathological polyspermic fertilization. This all together supports a new role of zinc ions during capacitation and fertilization. Such findings represent a fundamental shift in the understanding of mammalian fertilization, paving the way for a more accurate semen analysis to ameliorate the methodology of in vitro fertilization (IVF) and artificial insemination (Al).

(A comprehensive Materials and Methods is located on page 111.)

\subsection{Results}

We used state-of-the-art IBFC to characterize the sperm zinc signature using identical fluorescent probes as Chapter 2 [Zn-probe FluoZinn ${ }^{T M}-3$ AM (FZ3), DNA stain Hoechst 33342, and live/dead cell, plasma membrane-integrity reflecting exclusion DNA stain propidium iodide $(\mathrm{PI})$ ] paired with acrosomal remodeling 
detecting lectin PNA (Arachis hypogea/peanut agglutinin) conjugated to Alexa Fluor $^{\text {TM }} 647$ (PNA-AF647). The IBFC, which combines the fluorometric capabilities of conventional flow cytometry with high speed-multi-channel image acquisition, proved to be advantageous due to the high presence of $\mathrm{Zn}^{2+}$ in sperm cytoplasmic droplets and seminal debris, which otherwise would distort traditional flow cytometry results. We developed a unique gating and masking strategy to ensure unbiased data analysis (Fig. 3.1). Analyses were performed using the initial, presperm rich fraction of boar ejaculates, which had highest sperm viability/plasma membrane integrity, repeatability and sensitivity to proteasomal inhibition compared to secondary, sperm-rich fraction which appeared more prone to spontaneous capacitation and loss of plasma membrane integrity. All results are from boar spermatozoa unless otherwise specified.

\section{Zinc signature is indicative of capacitation status in vitro}

A drawback to commonly used $15 \mathrm{mM}$ sodium bicarbonate in vitro capacitation media is rapid sperm death [as compared to in vivo sequential capacitation (Jaiswal and Eisenbach, 2002)], illustrated in the time course study by a shift to $\mathrm{Pl}+$ cell death flow cytometry gating (Fig. 3.2a) and rapid acrosomal modification (Fig. 3.2b). In the interest of emulating in vivo sperm life span and sequential capacitation as a fertility diagnostic method, we used a previously described capacitation medium (Zimmerman et al., 2011) with low (2 mM) sodium bicarbonate and increased sodium pyruvate $(5 \mathrm{mM})$ that prolonged sperm viability (Fig. 3.2c) and elicited similar hyperactivation (Movie 3.1, link) while achieving 
hallmark acrosomal modification (Fig. 3.2d; discussed further in Materials \& Methods, in vitro capacitation section). These IVC conditions had no difference in relative protein tyrosine phosphorylation as detected by Western blotting (Fig. 3.2e).

Most spermatozoa in zinc signature 1 and 2 states had no capacitation-like acrosomal remodeling $(93.0 \% \pm 6.8 \%$ and $95.0 \% \pm 2.6 \%$, data presented as mean \pm s.d.; 10,000 cells analyzed per treatment, $n=3$ biological replicates) compared to zinc signature 3 and $4(11.1 \% \pm 5.8 \%$ and $7.0 \% \pm 9.9 \% ; P<0.0001$, as determined by the General Linear Model (GLM) procedure). Capacitation-like acrosomal remodeling was most prevalent with zinc signatures 3 and $4(81.0 \% \pm 8.5 \%$ and $62.2 \% \pm 12.9 \%)$ compared to zinc signatures 1 and $2(4.0 \% \pm 4.7 \%$ and $3.4 \% \pm$ $2.9 \% ; P<0.0001$, as determined by the GLM procedure). Acrosome exocytosis occurred within the subpopulation of spermatozoa with zinc signature $4(30.7 \% \pm$ $3.0 \%)$ and was greater than zinc signatures 1,2 , and $3(3.0 \% \pm 2.6 \%, 1.6 \% \pm 1.2 \%$, $7.9 \% \pm 2.9 \% ; P<0.001$, as determined by the GLM procedure; Fig. 3.3a; Table 3.1). As sperm plasma membrane-integrity decreased, signaled by increased propidium iodide $(\mathrm{PI})$ labeling, the zinc patterns progressed to signatures 3 and 4 (Fig. 3.3b). Coincidentally, as sperm plasma membrane-integrity decreased, acrosomal remodeling and exocytosis occurred (Fig. 3.3c). Hyperactivated spermatozoa, capable of recognizing and binding the oocyte zona pellucida have zinc signature 2 (Movies 3.2-4, individual hyperlinks located in movie legends), in 
which the transition from signature 1 to 2 occurs within the first $30-60$ minutes of IVC (Fig. 3.3d).

\section{S proteasome modulates Zinc signature capacitation shift}

Fresh, ejaculated boar spermatozoa mostly had signature $1,(83.8 \% \pm 3.1 \%$; data presented as mean \pm s.e.m.; 10,000 cells analyzed per treatment, $n=3$ biological replicates; Fig. 3.4a \& i; Table 3.2). A portion of spermatozoa incubated in nonIVC media for 4 hours at $37^{\circ} \mathrm{C}$ progressed to signature 2 (Fig. 3.4b) as compared to spermatozoa in the same media incubated at room temperature to emulate the conditions of artificial insemination (Fig 3.4a), suggesting that some spermatozoa undergo temperature-induced, early stage capacitation. When proteasome inhibitor MG132 was added to IVC conditions to reduce sperm proteasome activity as previously described (Yi et al., 2009; Miles et al., 2013), a significantly higher portion of spermatozoa retained signature 1 when using the pre-sperm rich fraction (Fig. 3.4c and i) and as compared to IVC + vehicle (Fig. 3.4e and i, $P=0.0271$; when signatures $1 \& 2$ combined $P=0.0008$, as determined by Duncan's Multiple Range test). After 4 hours of IVC, the zinc signature changed to mostly signature $3(49.4 \% \pm 7.9 \%)$, with a small portion of spermatozoa having signature $2(31.3 \%$ $\pm 12.3 \%$; Fig. $\mathbf{3 . 4 g}$ ). Spermatozoa retained signature 1 with addition of $1 \mathrm{mM} \mathrm{ZnCl} 2$ to IVC medium (Fig. 3.4h). A similar shift towards signature 3 was seen in human spermatozoa after 4 hours of IVC (Fig. 3.5). 


\section{Zinc signature associated with varied fertility in Al boars.}

We examined possible individual variability in sperm zinc signature in Al boars with varied fertility. In a small preliminary fertility trial $(n=4$ boars with known fertility in Al service; fertility records in Table 3.3), zinc signatures differed between high and low fertile boars after IVC (Fig. 3.6a). Boars with high fertility had double the amount of signature 3 spermatozoa prevail after IVC (as percentage of population) as opposed to minimal signature 3 increase in low fertility boars (Fig. 3.6b).

\subsection{Discussion}

The inhibition of the zinc signature shifts may be related to the presence of $\mathrm{Zn}$ binding UPS enzymes in spermatozoa, such as the RING-finger E3-type ubiquitin ligase UBR7 (Zimmerman et al., 2014). The RING (Really Interesting New Gene) finger ubiquitin ligases contain an amino acid motif that binds two zinc cations, allowing interaction with other proteins/enzymes, including establishment of a stable E3-substrate interaction required for protein ubiquitination. Other $\mathrm{Zn}$ binding/Zn-containing proteins are likely present in spermatozoa since UBR7 is only detectable in the acrosomal region of fully differentiated spermatozoa and in the centriolar vault of the sperm tail-connecting piece. For example, sperm DNAbinding protamine PRM2 contains a zinc-finger domain (Bianchi et al., 1992), which plays a role in stabilization of sperm chromatin and inhibition of transcription.

Protamines are believed essential for sperm head condensation and DNA stabilization during spermiogenesis, and alterations in sperm DNA protamination are associated with male infertility (Nanassy et al., 2011). Further, JAMM 
(JAB1/MPN/Mov34 metalloenzyme) motif of the proteasomal regulatory subunit PSMD14/Rpn11, is a metalloprotease-like $\mathrm{Zn}$ site in the $26 \mathrm{~S}$ proteasome (Ambroggio et al., 2003). Matrix metalloproteinases are zinc-dependent enzymes known mainly for their ability to digest extracellar matrix (ECM) (Nagase and Woessner, 1999), with MMP2 and MMP9 being reported in human seminal plasma (Shimokawa Ki et al., 2002). Inhibition of zinc-dependent metalloproteases hindered sperm passage through oocyte vestments during IVF (Beek et al., 2012). Related to aforementioned sperm chromatin stabilization by protamination, $\mathrm{Zn}^{2+}$ release from the nuclear zinc bridges described in human spermatozoa could facilitate sperm nucleus decondensation after fertilization, as a prelude to the formation of the zygotic paternal pronucleus (Kvist, 1980a; Bjorndahl and Kvist, 2010). Further studies thus should examine the relationship between sperm zinc signature and the aforementioned Zn-containing sperm proteins.

The changes seen in fresh, ejaculated zinc signature and the incubated, non-IVC spermatozoa could be heat-induced during the early stages of capacitation. Temperature influence on capacitation state could be related to the $35-38^{\circ} \mathrm{C}$ thermotropic phase transition identified in the plasma membrane of ram spermatozoa (Holt and North, 1986). These findings are important for livestock semen handling methods prior to $\mathrm{Al}$.

Sperm capacitation, although required for fertility, is a terminal maturation event that leads to rapid cell death unless fertilization occurs (Jaiswal and Eisenbach, 
2002). The superimposition of zinc ion labeling and PI labeling in flow cytometric scatter plots allows us to subdivide spermatozoa within the boar ejaculate into four subpopulations (Fig. 3.3b). Thus, the disproportional representation of signatures 3 and 4, associated with sperm capacitated state and death, may indicate low fertility ejaculates. Cell membrane changes heralded by PI incorporation in the sperm head at capacitation are concomitant with acrosomal remodeling signaled by lectin PNA binding, even though they occur at the opposite poles of the sperm head (Fig. 3.3c). As indicated in our time lapse study, PI intensity changes over the course of capacitation and there are two subgroups of PI+ spermatozoa: $\mathrm{PI}+$ live with plasma membrane change and $\mathrm{PI}+$ cell death (Fig. 3.2a and c). Although the signature 2 spermatozoa are the hyperactivated and zona pellucida-interacting ones in Movie 3.4 (link), it is likely that a rapid transition occurs through these last stages of capacitation that cannot be distinguished within limitations of today's technology. After this rapid transition from zinc signature 2 to 3 and associated acrosomal changes, we hypothesize that the final cell death occurs rapidly if fertilization is unsuccessful. This might be the necessitating reason for sequential sperm capacitation observed within the oviductal sperm reservoir.

Comparison of zinc signature patterns in boars with varied fertility indicates potential of $\mathrm{Zn}$ probes in the evaluation of livestock sperm quality. While such findings with a small group of boars are preliminary, $\mathrm{Zn}$ fluorometry could be also given consideration in human andrology and infertility diagnostics. For instance, the sperm content of flagellar voltage-gated proton channel HVCN1 varies 
between human donors (Lishko et al., 2010). The HVCN1 regulates human CatSper $\mathrm{Ca}^{2+}$ channel localized at the flagellum (Lishko et al., 2010). With CatSper activation required for hyperactivation and ultimately male fertility (Qi et al., 2007), it seems reasonable that zinc signature changes are reflective of this biological event and necessary for preparing the spermatozoa for hyperactivation. Other studies have shown that spermatozoa with higher fertilizing ability have increased changes in capacitation-induced biomarkers (Buffone et al., 2005). Altogether, this encourages dedicated trials with high statistical power sperm sample sets, aspiring to validate zinc signature as a candidate fertility marker. Such findings not only indicate the existence of sperm subpopulations capable/incapable of fertilizing the oocyte, but even more so that sequential capacitation and resulting waves of sperm release from the sperm reservoir, originally thought to be primarily driven by female reproductive tract-issued signals (Giojalas et al., 2004; Suarez, 2008; Leemans et al., 2015), are rather co-dependent of sperm sub-population (Fig. 3.7a and b). Other recent studies hint at the significance of $\mathrm{Zn}^{2+}$ for sperm structure and function/fertility. A decrease in $\mathrm{Zn}^{2+}$ content of human seminal plasma has been associated with infertility stemming from accidental Chernobyl radiation in Ukraine (Andreychenko et al., 2016) and individuals with high levels of asthenoteratozoospermic spermatozoa (low or no motility) have reduced seminal plasma $\mathrm{Zn}^{2+}$ levels (Nenkova et al., 2017). These findings are consistent with other studies reporting fertile males having increased seminal plasma $\mathrm{Zn}^{2+}$ levels compared to infertile men (Colagar et al., 2009; Zhao et al., 2016). Optimization of 
semen $\mathrm{Zn}^{2+}$ and/or zinc containing protein(s) levels could thus improve the outcomes of $\mathrm{Al}$ in livestock and assisted reproductive therapy in humans.

The signature we describe here is likely representative of $\mathrm{Zn}^{2+}$ being involved in multiple steps of sperm capacitation. At ejaculation, sperm motility is highly dependent upon ionic environment (Harrison et al., 1978). It is well understood that $\mathrm{Zn}^{2+}$ can modulate cellular signaling, as well as protein kinase and phosphatase activities (Beyersmann and Haase, 2001), and inhibit proteasomal activity (Kim et

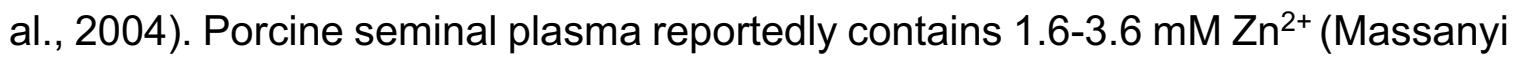
et al., 2003), the highest known levels of $\mathrm{Zn}^{2+}$ found in any bodily fluid, thus likely to be serving some biological function. Further, Zn-binding seminal plasma proteins may protect the sperm plasma membrane against cold shock (Mogielnicka-Brzozowska et al., 2011). As mentioned earlier, $\mathrm{Zn}^{2+}$ negatively regulates HVCN1 channel, the main proton extrusion mechanism in human spermatozoa (Lishko et al., 2010). Certain aspects of early capacitation events such as $\mathrm{Ca}^{2+}$ influx require HVCN1 activation by $\mathrm{Zn}^{2+}$ removal. The $\mathrm{Zn}^{2+}$ content in the human sperm flagella is negatively correlated to sperm motility (Henkel et al., 1999); however, this may be due to capacitation-induced hyperactivation being inhibited in the presence of high $\mathrm{Zn}^{2+}$. Further, $\mathrm{Zn}$-chelator DEDTC has been shown to immobilize spermatozoa, reaffirming the role of $\mathrm{Zn}^{2+}$ in sperm motility (Sørensen et al., 1999). Beyond sperm capacitation, zinc signature or sperm zinc signaling of spermatozoa bound to the oocyte zona pellucida could be altered by the zinc spark (Que et al., 2015; Zhang et al., 2016) triggered by the first fertilizing 
spermatozoon as well be altered by the $300 \%$ increase of zinc content in the zona pellucida (ZP) matrix following the zinc spark (Que et al., 2017). Altogether, such massive release of extracellular $\mathrm{Zn}^{2+}$ and increased $\mathrm{ZP} \mathrm{Zn}^{2+}$ content could establish a combined zinc shield. Consequently this zinc shield could derail Znsignaling in the spermatozoa surrounding the oocyte as an added barrier to polyspermic fertilization. Such mechanism seems plausible in consideration that $\mathrm{Zn}^{2+}$ has been shown to be chemorepulsive to fertilization-competent human, mouse, and rabbit spermatozoa (Guidobaldi et al., 2017). Furthermore, acrosin and matrix metalloproteinase MMP2, two of the proteinases implicated in spermzona pellucida penetration, are present in the inner acrosomal membrane [(Ferrer et al., 2012a), the location believed to be the leading edge of the sperm head during ZP penetration], have been shown to have their activities reduced and/or inhibited by $\mathrm{Zn}^{2+}$ [human and bovine sperm acrosin (Steven et al., 1982), paddlefish acrosin-like activity (Ciereszko et al., 2000), and brain MMP2 activity (Backstrom et al., 1992)]. Increased $\mathrm{Zn}^{2+}$ concentration in bovine IVF media has previously been shown to inhibit fertilization (Stephenson and Brackett, 1999) adding further support to the effective role of the zinc shield. Based on the present data, sperm zinc signature likely changes as the spermatozoa advance through the female reproductive tract and progress through different stages of capacitation; the proposed, reciprocal sperm and oocyte $\mathrm{Zn}^{2+}$-signaling for the blockage of polyspermy is outlined in Fig. 3.7b. Lastly, porcine ZP glycoproteins are highly glycosylated with neutral and acidic $\mathrm{N}$ - and O-linked oligosaccharides. N-glycans released from the ZP3-glycoprotein mixture are composed of neutral and acidic 
structures in approximate molar ratio of 1:2 (Noguchi and Nakano, 1992). While neutral zona N-glycans have been found to be the determining sugar signals in the pig (Yurewicz et al., 1991; Noguchi et al., 1992), little is known about the function of acidic moieties of ZP glycoproteins (Amari et al., 2001). It is plausible that $\mathrm{Zn}^{2+}$, after $\mathrm{Zn}^{2+}$ efflux associated with gamete fusion, may electrostatically bind to sialic residues of acidic oligosaccharides of $\mathrm{ZP}$, altering ZP polarity and thus act as a fast block of polyspermy on the egg coat level by creating a zinc shield at the time of sperm-oolemma fusion. A similar effect could be achieved by $\mathrm{Zn}^{2+}$ on the sperm surface, but most probably via a different mechanism, such as conformational change of ZP receptors resulting in decreased sperm affinity for the ZP.

Such findings shift the paradigm of anti-polyspermy defense mechanisms and complement the discovery of oocyte zinc spark. Herein, if capacitation is defined solely as the capacity to fertilize, it would seem reasonable that the oocyte can decapacitate spermatozoa to some degree by manipulating sperm Zn-levels; however, direct relationship with zinc signature should be explored.

In conclusion, the novel biological phenomenon of sperm zinc signature is an early indicator of sperm capacitation and a candidate biomarker of sperm quality/fertility. The sperm proteasome, at least in the sub-population of capacitation competent spermatozoa, co-regulates earlier events of sperm capacitation than originally realized. The study of sperm zinc signature may further enhance our understanding of fertilization, including but not limited to the oocyte zinc shield 
formed upon fertilization to prevent polyspermy and sperm subpopulations capable of fertilization.

\subsection{Acknowledgements}

Supported by the National Institute of Food and Agriculture (NIFA), U.S. Department of Agriculture (USDA) grant number 2015-67015-23231 (PS), USDA NIFA Graduate Fellowship award number 2017-67011-26023 (KK), grant number 5 R01 HD084353-02 from NIH National Institute of Child and Human Development (PS), European Regional Development Fund (ERDF) BIOCEV grant CZ.1.05/1.1.00/02.0109 (MZ), the Czech Academy of Sciences (RVO:866525036) (MZ) and seed funding from the Food for the $21^{\text {st }}$ Century Program of the University of Missouri (PS). We thank staff of the National Swine Research and Resource Center, University of Missouri, as well as Dr. Randall Prather and his associates for wild-type boar semen collection, funded by National Institutes of Health $(\mathrm{NIH})$ U42 OD011140. We also thank Dr. Michal Zigo for helping with various aspects of the study, Mr. Dalen Zuidema for providing MII porcine oocytes, and Ms. Katherine Craighead for clerical support. 
Gating approach

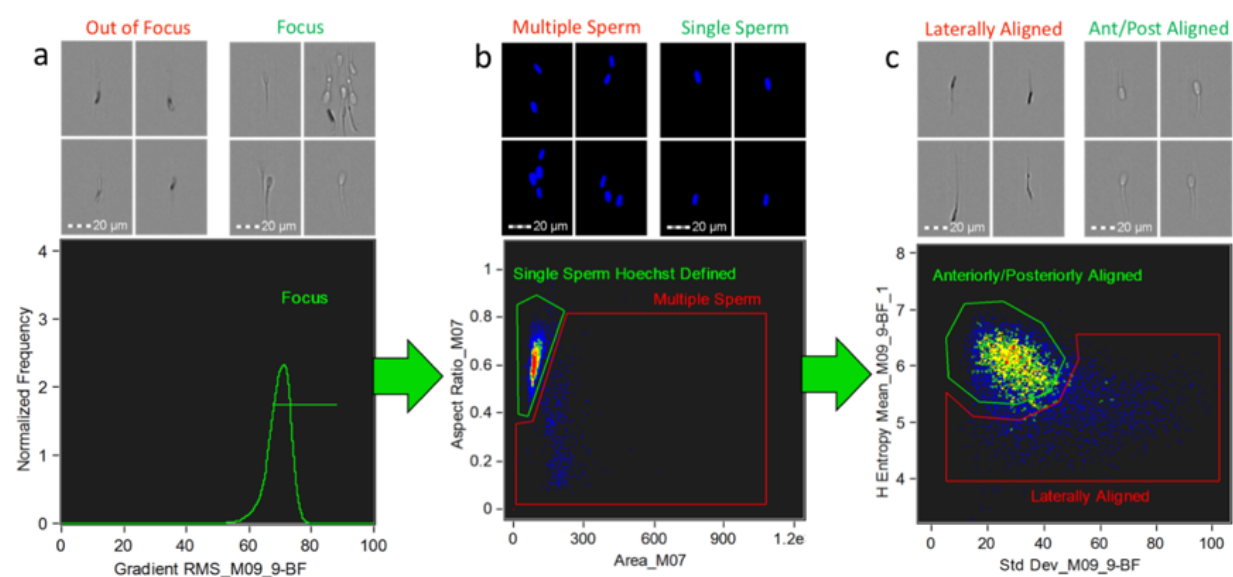

Masking approach

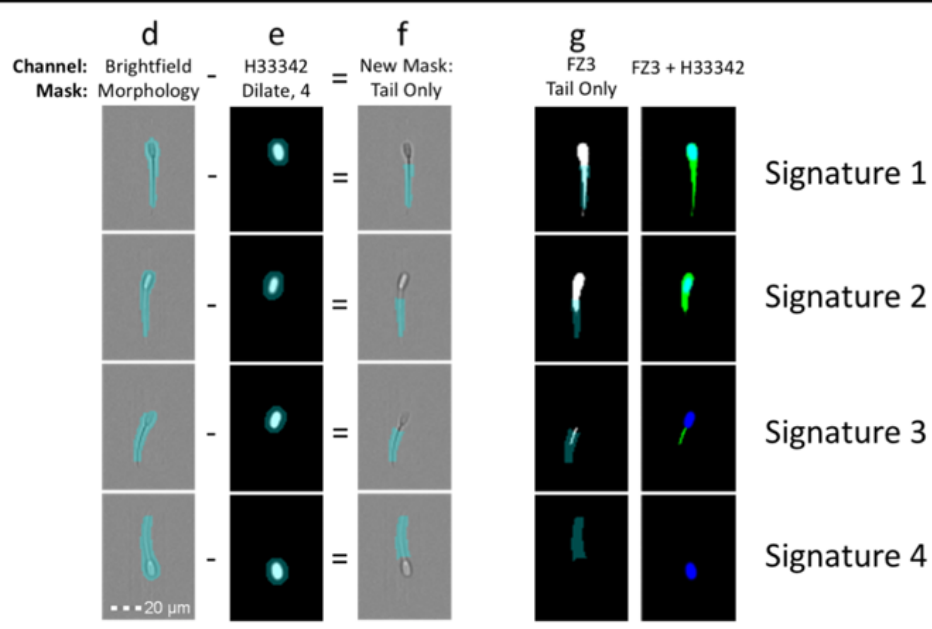

Gating and masking approach combined
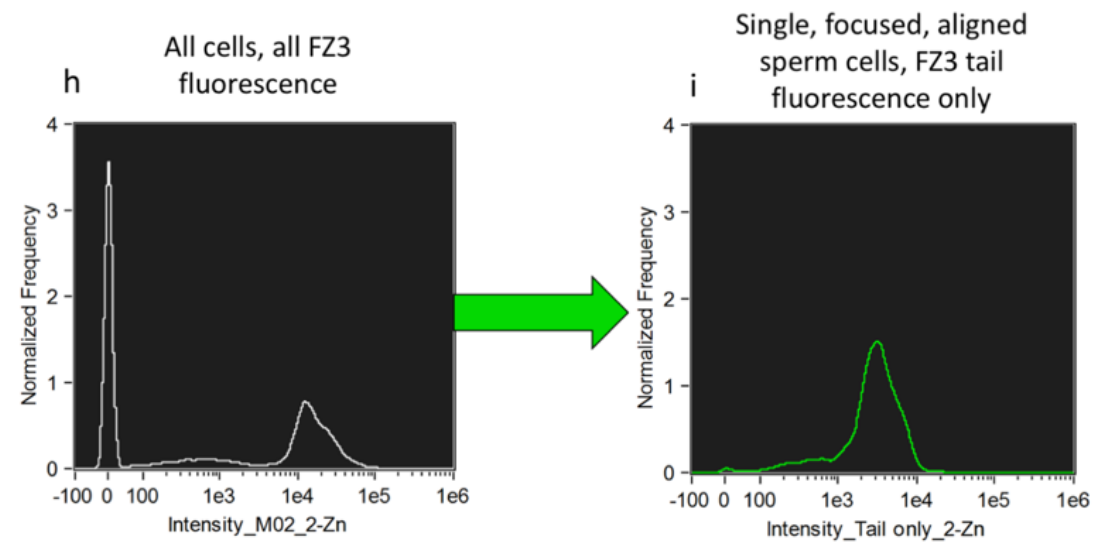

Figure 3.1. Gating and masking approach. a, Initial events collected from FlowSight data acquisition were gated for cells in focus as a function of brightfield 
gradient RMS, (a calculation of image crispness). b. Events in focus are further gated to analyze only single spermatozoa, plotted as H33342 fluorescence area by aspect ratio. c, To differentiate and gate out laterally aligned spermatozoa, a plot of brightfield standard deviation by brightfield $\mathrm{H}$ entropy mean is used. $\mathbf{d}-\mathbf{g}$, Mask (cyan) defines the region analyzed; fluorescence color removed to create greater contrast. d, Morphology mask of the brightfield e, subtracting a 4 pixel dilation of $\mathrm{H} 33342 \mathbf{f}$, resulting in mask analyzing $\mathrm{FZ3}$ fluorescence in the sperm tail only. $\mathbf{h}$, Conventional flow cytometry (FC) Zn-probe histogram compared to resulting gating and masking strategy (i), ensuring prudent data analysis not possible with conventional FC (i; all scale bars: $20 \mu \mathrm{m}$ ). 


\section{$15 \mathrm{mM} \mathrm{NaHCO}_{3}$ IVC Media Time Course}
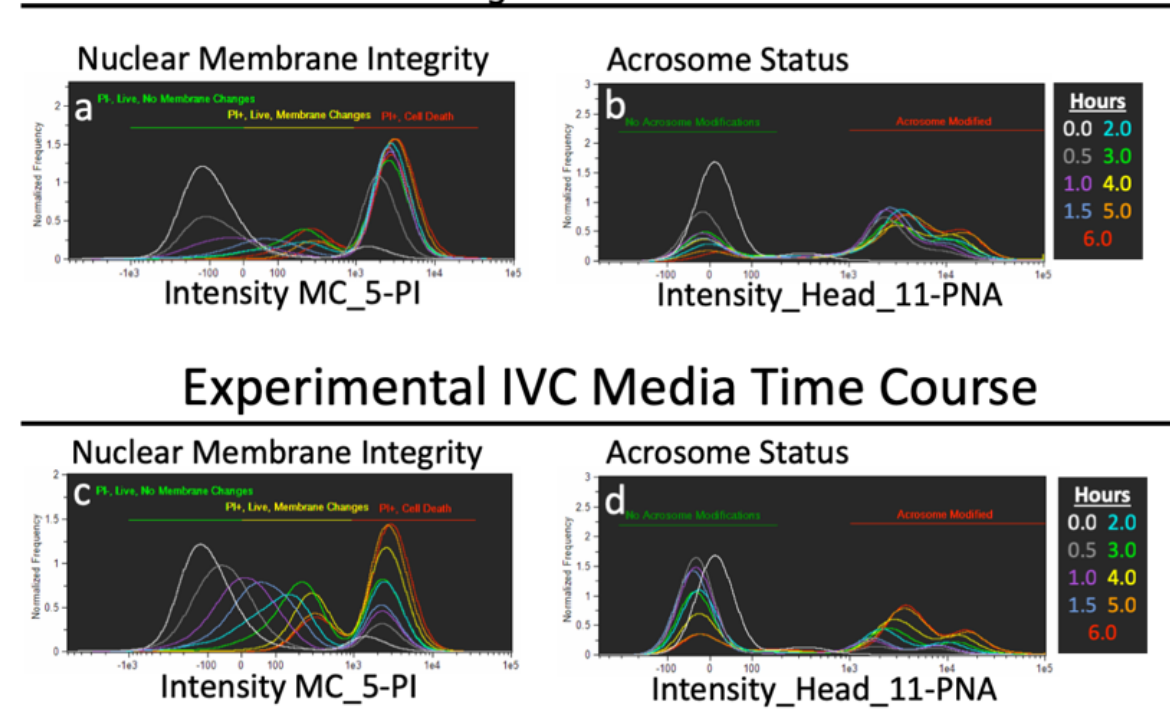

\section{Confirmation of IVC: tyrosine phosphorylation}

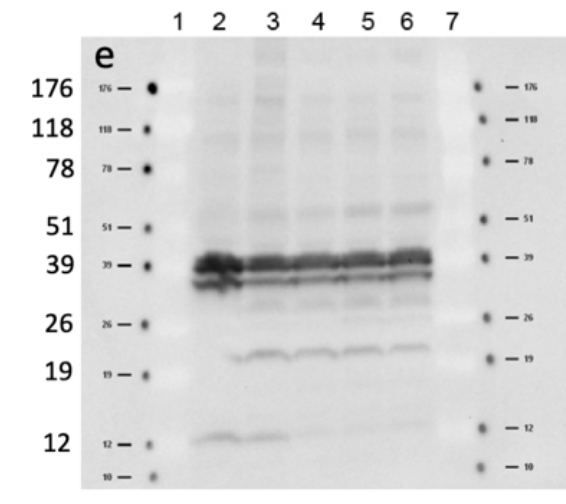

Anti-pY

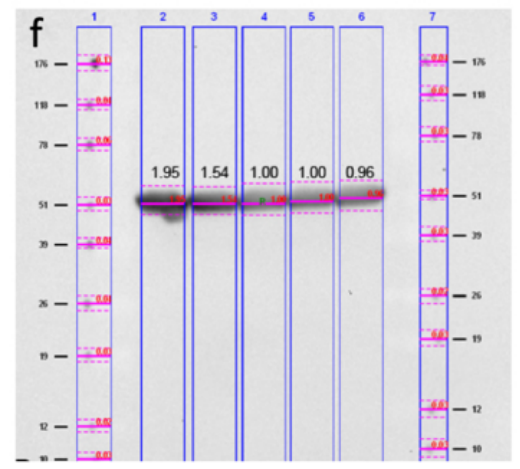

Anti-tubulin

Figure 3.2: Capacitation status confirmation under high versus low bicarbonate IVC conditions. Time course capacitation status confirmation in high, $15 \mathrm{mM}$ sodium bicarbonate media: a, Plasma membrane changes as identified by PI status, distinguishing between IVC-induced $\mathrm{PI}+$ subpopulations ( $\mathrm{PI}+$ live with plasma membrane changes vs $\mathrm{PI}+$ cell death). $\mathbf{b}$, Acrosomal modifications. Time course capacitation status confirmation in experimental IVC (low, $2 \mathrm{mM}$ sodium bicarbonate, $5 \mathrm{mM}$ pyruvate): c, Plasma membrane changes, and $\mathbf{d}$, acrosomal modifications (corresponding histogram color code for time points in figure legend). 
e-f, Anti-phosphotyrosine Western blot of sperm extracts from treatments in the following lanes: 1) marker; 2) ejaculated, non-IVC; 3) experimental IVC conditions (2 mM sodium bicarbonate, $5 \mathrm{mM}$ pyruvate); 4) comparison IVC (15 mM sodium bicarbonate, $0.2 \mathrm{mM}$ pyruvate); 5) comparison IVC (15 mM sodium bicarbonate, 5 mM pyruvate). The density of band migrating between 19-26 kDa markers (panel e) remained unchanged relative to loading control (panel f) in the extracts of spermatozoa capacitated with high (lane 4) vs. low sodium bicarbonate and pyruvate (lane 3). f, Loading control anti-tubulin Western blot with quantification for normalization. 


\section{Porcine}
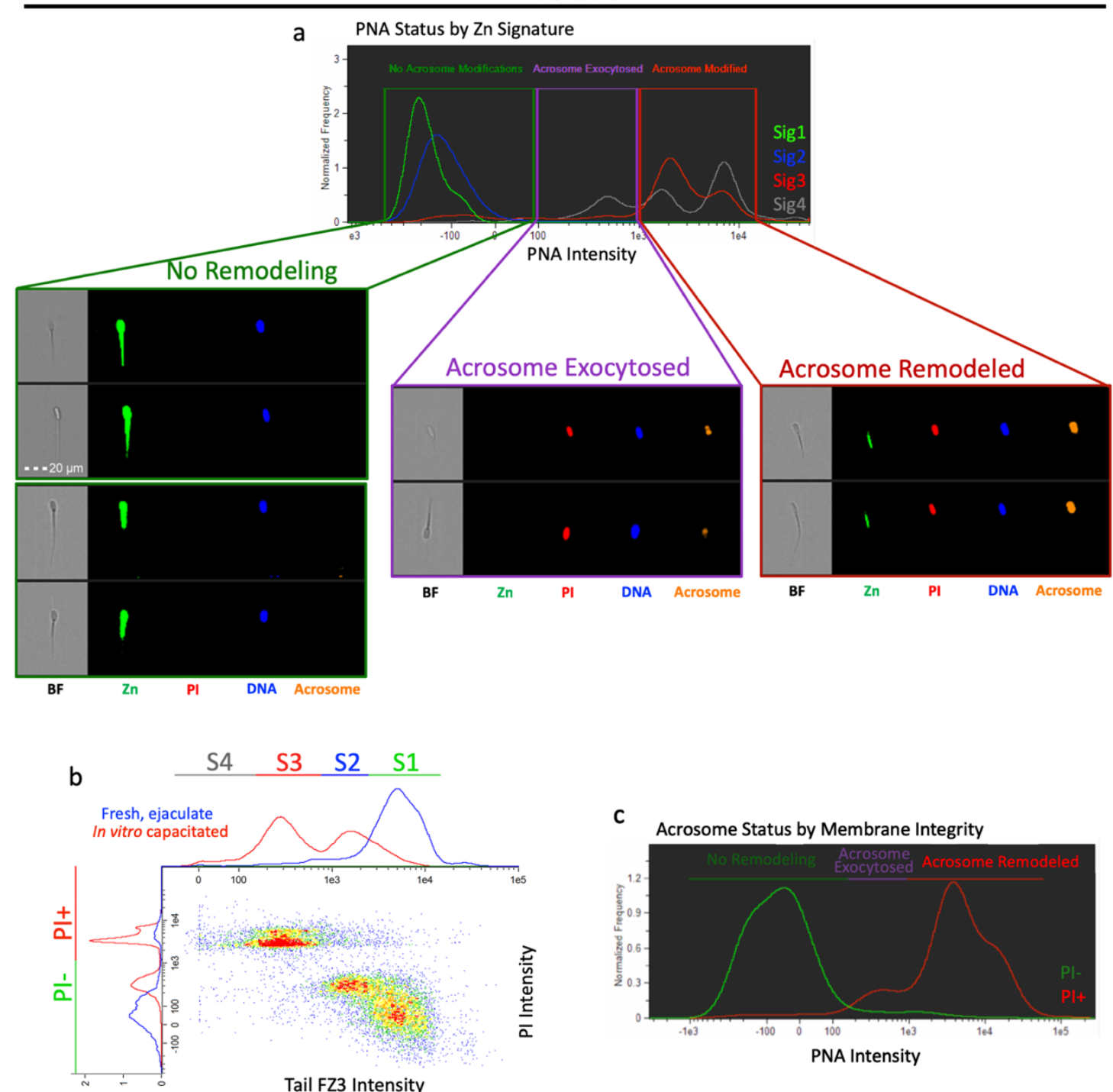

Tail FZ3 Intensity

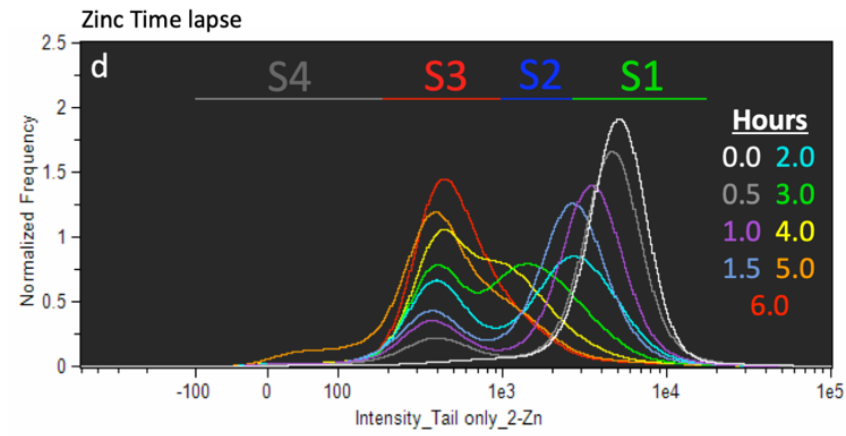

Figure 3.3: Acrosomal status and membrane integrity of zinc signature. a, Most spermatozoa in zinc signature 1 and 2 states had no capacitation-like acrosomal 
remodeling compared to zinc signature 3 and $4(P<0.0001$; see Table 3.1). Capacitation-like acrosomal remodeling was most prevalent with zinc signatures 3 and 4 compared to zinc signatures 1 and $2(P<0.0001 ; 4$ biological replicates; 10,000 spermatozoa analyzed per treatment). Acrosomal exocytosis occurred within the subpopulation of spermatozoa with zinc signature 4 and was greater than zinc signatures 1,2 , and $3(P<0.001$; scale bar: $20 \mu \mathrm{m})$. b, Zinc signature status corresponds with PI plasma membrane integrity in fresh (blue) and IVC spermatozoa (red). c, As sperm plasma membrane-integrity decreased, acrosomal remodeling and exocytosis occurred. $\mathbf{d}$, Zinc signature IVC time course (corresponding histogram color code for time points is embedded in figure panel). $P$-values determined by the General Linear Model procedure in SAS 9.4. 
Porcine

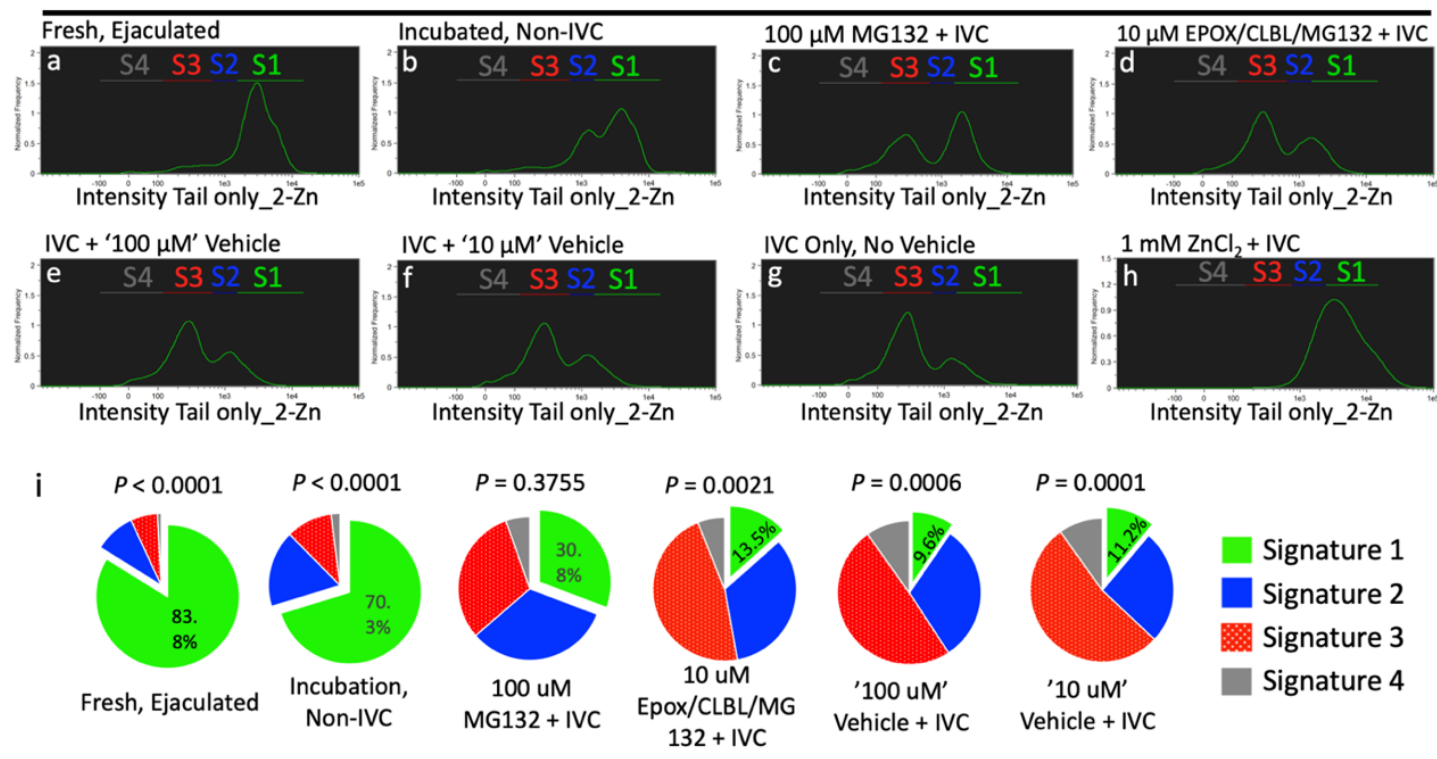

Figure 3.4: Modulation of internal and external $\mathrm{Zn}^{2+}$ during IVC. a, Fresh, ejaculated spermatozoa have mostly signature 1. b, After 4 hours under non-IVC conditions, few spermatozoa underwent spontaneous early stage capacitation. c, Proteasomal inhibitor MG132 (100 $\mu \mathrm{M})$ and proteasomal inhibitor cocktail composed of MG132, Epoxomycin and CLBL (10 $\mu \mathrm{M}$; d) prevented some of IVCinduced zinc signature changes compared to $\mathbf{e}$ and $\mathbf{f}$ (IVC with appropriate vehicle); g, No vehicle IVC control. h, Addition of $1 \mathrm{mM} \mathrm{ZnCl}_{2}+$ IVC prevented IVC-induced zinc signature changes. i, Pie chart illustration of select IVC treatments (green: signature 1; blue: signature 2; red: signature 3; gray: signature 4; $P$-value by treatment across replicates). Treatment $\mathrm{P}$-values are shown in Table 3.2 as determined by the General Linear Model procedure in SAS 9.4. 10,000 sperm per sample analyzed. 


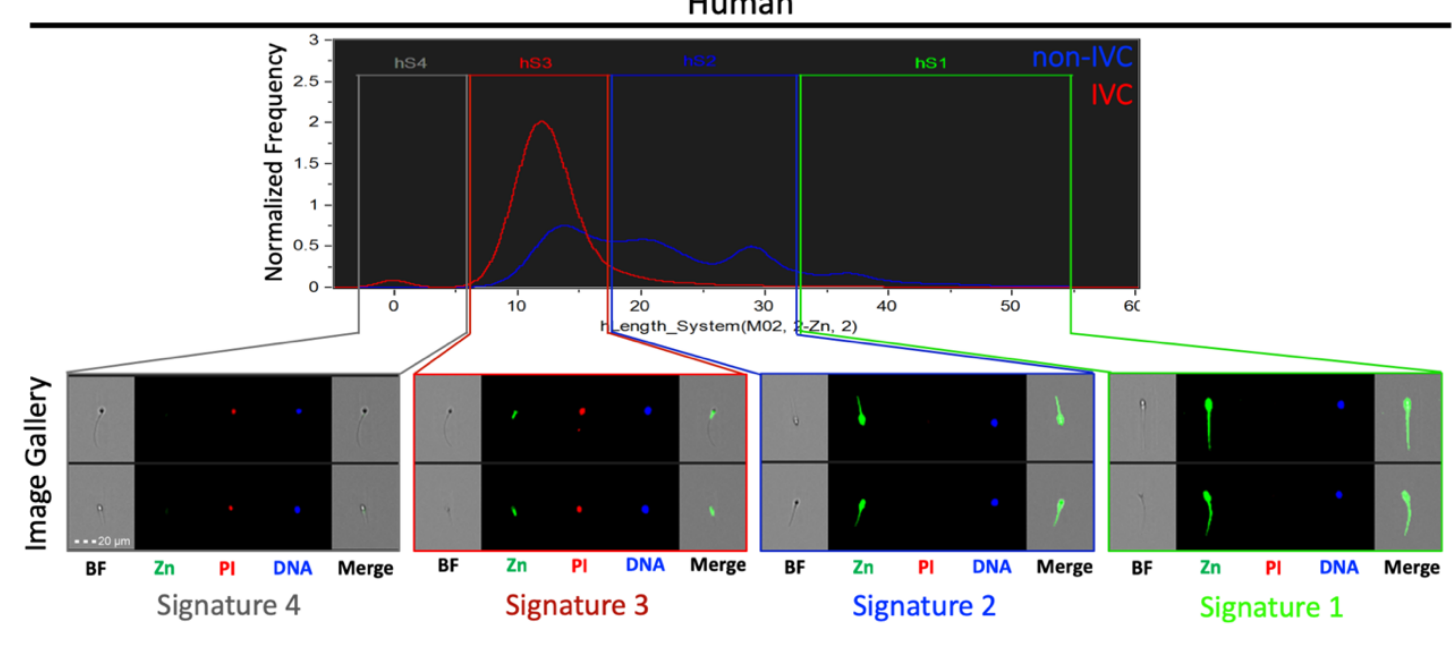

Figure 3.5: Zinc signature of human spermatozoa after IVC. Human spermatozoa undergo shift similar to porcine spermatozoa, towards signature 3 after 4 hours of IVC (histogram, red) compared to neat, non-IVC spermatozoa (histogram blue; scale bar: $20 \mu \mathrm{m})$. 


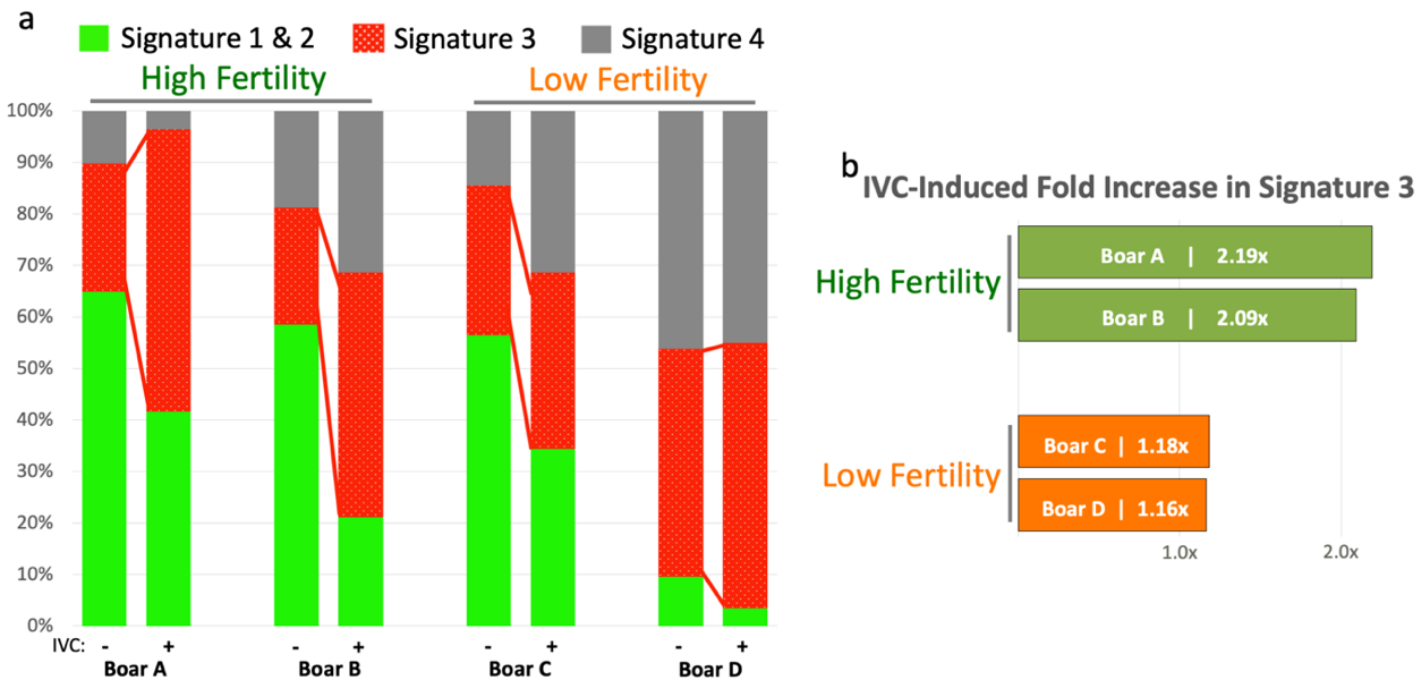

Figure 3.6. Zinc signature boar fertility trial. a, Zinc signature in four boars of known high or low fertility, before and after IVC. b, High fertility boars had double the amount of spermatozoa with signature 3 after IVC compared to minimal increase in low fertility boars. Fertility records located in Table 3.3. 


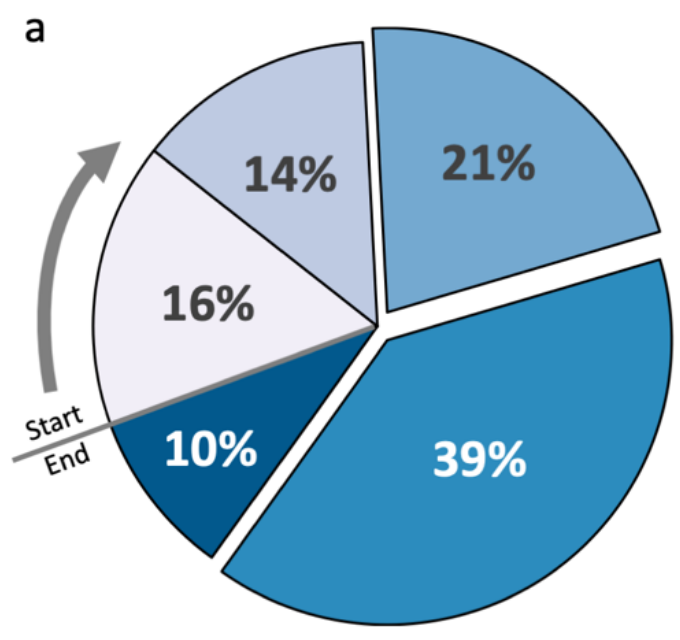

Key

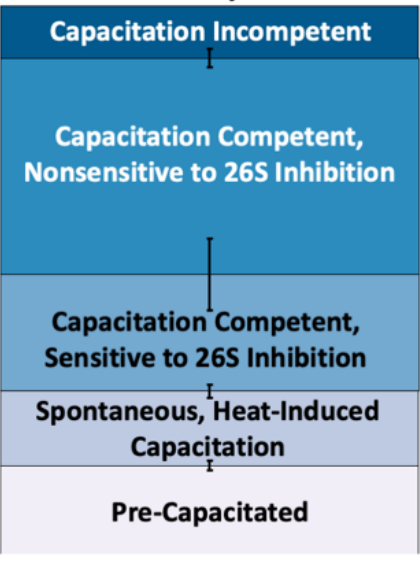

b
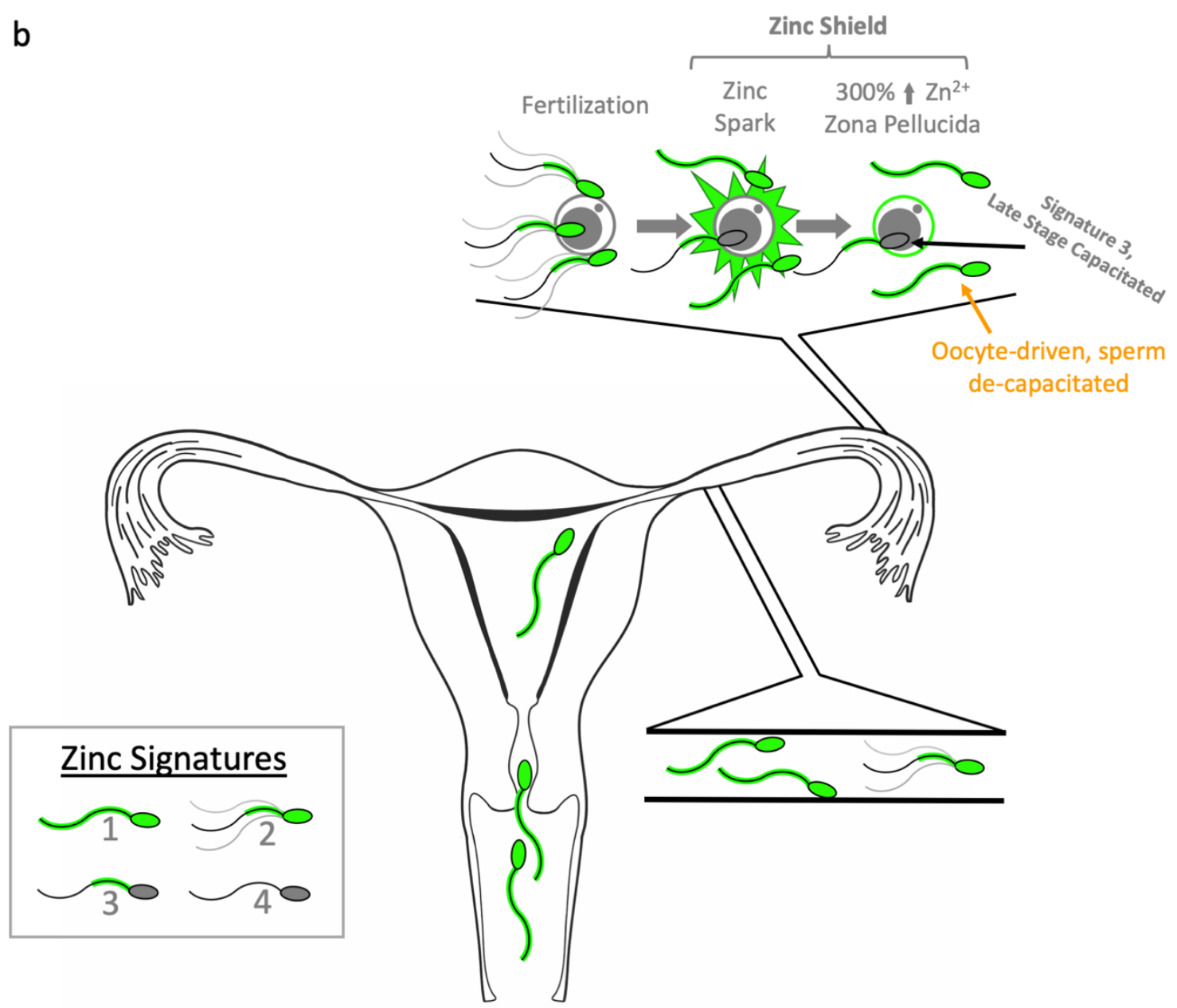

Figure 3.7. Proposed zinc signature population interpretation. a, Interpretation of zinc signature meaning and population segregation: $16 \%$ of fresh, ejaculated 
spermatozoa had undergone early stage capacitation upon semen collection (lightest blue working to darkest); $14 \%$ of spermatozoa spontaneously undergo early stage capacitation during incubation without IVC-inducers; $60 \%$ of spermatozoa remained capacitation competent with IVC-inducers, with $21 \%$ sensitive to proteasomal inhibition; remaining $10 \%$ of sperm were capacitation incompetent under IVC-conditions (darkest blue; standard error bars included). b, Proposed zinc signature changes throughout female reproductive tract and oocyte zinc spark interference with sperm zinc signature as a combined polyspermy defense mechanism, the zinc shield. 


\begin{tabular}{|l|c|c|c|}
\hline & No Remodeling & Remodeled & Exocytosed \\
\hline Signature 1 & $93.0 \% \pm 6.8 \%^{\mathrm{Aa}}$ & $4.0 \% \pm 4.7 \%{ }^{\mathrm{Ba}}$ & $3.0 \% \pm 2.6 \%^{\mathrm{Ba}}$ \\
\hline Signature 2 & $95.0 \% \pm 2.6 \%^{\mathrm{Aa}}$ & $3.4 \% \pm 2.9 \%^{\mathrm{Ba}}$ & $1.6 \% \pm 1.2 \%^{\mathrm{Ca}}$ \\
\hline Signature 3 & $11.1 \% \pm 5.8 \%^{\mathrm{Ab}}$ & $81.0 \% \pm 8.5 \%^{\mathrm{Bb}}$ & $7.9 \% \pm 2.9 \%^{\mathrm{Aa}}$ \\
\hline Signature 4 & $7.0 \% \pm 9.9 \%^{\mathrm{Ab}}$ & $62.2 \% \pm 12.9 \%^{\mathrm{Bb}}$ & $30.7 \% \pm 3 . \%^{\mathrm{Cb}}$ \\
\hline
\end{tabular}

Table 3.1. Statistical analysis of zinc signature and acrosomal status. Data are presented as mean \pm s.d. ( 3 biological replicates). Values with different uppercase superscripts $(A, B, C, D)$ indicate significant difference of the acrosomal status ( $P$-value $\leq 0.0001)$ and lowercase superscripts $(\mathrm{a}, \mathrm{b}, \mathrm{c})$ indicate significant difference of zinc signatures $(P$-value $\leq 0.0002)$ as determined by the General Linear Model procedure in SAS 9.4. Both $\mathrm{PI}+$ and $\mathrm{PI}$ - cells were included in this analysis. A total of 10,000 cells were measured for each replicate. 


\begin{tabular}{|c|c|c|c|c|c|}
\hline Treatment & $\begin{array}{c}\text { Signature } \\
1\end{array}$ & $\begin{array}{c}\text { Signature } \\
2\end{array}$ & $\begin{array}{c}\text { Signature } \\
3\end{array}$ & $\begin{array}{c}\text { Signature } \\
4\end{array}$ & $P$-value \\
\hline $\begin{array}{r}\text { Fresh, } \\
\text { Ejaculated }\end{array}$ & $\begin{array}{c}83.8 \% \\
\pm 1.8 \% \mathrm{Aa}\end{array}$ & $\begin{array}{c}9.2 \% \\
\pm 0.9 \% \mathrm{~b}\end{array}$ & $\begin{aligned} & 6.1 \% \\
& \pm 1.2 \% \\
&\end{aligned}$ & $\begin{aligned} & 0.9 \% \\
\pm & 0.4 \% \mathrm{Ac}\end{aligned}$ & $\begin{array}{c}P< \\
0.0001\end{array}$ \\
\hline $\begin{array}{r}\text { Incubation, } \\
\text { Non-IVC }\end{array}$ & $\begin{array}{c}70.3 \% \\
\pm 2.5 \% \mathrm{Aa} \\
\end{array}$ & $\begin{array}{c}17.3 \% \\
\pm 3.0 \% \\
\end{array}$ & $\begin{array}{c}10.4 \% \\
\pm 2.7 \% \mathrm{Ab} \\
\end{array}$ & $\begin{aligned} & 2.0 \% \\
& \pm 2.7 \% \mathrm{Ac} \\
&\end{aligned}$ & $\begin{array}{c}P< \\
0.0001\end{array}$ \\
\hline $\begin{array}{r}100 \mu M \\
M G 132+I V C \\
\end{array}$ & $\begin{array}{c}30.8 \% \\
\pm 13.1 \%{ }^{\mathrm{B}} \\
\end{array}$ & $\begin{array}{r}32.9 \% \\
\pm 19.5 \% \\
\end{array}$ & $\begin{array}{c}31.0 \% \\
\pm 4.9 \% \\
\end{array}$ & $\begin{aligned} & 5.4 \% \\
& \pm 4.9 \% \mathrm{AB} \\
&\end{aligned}$ & $\begin{array}{c}P= \\
0.3755\end{array}$ \\
\hline $\begin{array}{r}10 \mu M \text { Epox, } \\
\text { CLBL, MG132 } \\
+ \text { IVC }\end{array}$ & $\begin{array}{c}13.5 \% \\
\pm 4.0 \% \mathrm{BC}\end{array}$ & $\begin{array}{l}33.7 \% \\
\pm 8.8 \%\end{array}$ & $\begin{array}{c}46.8 \% \\
\pm 3.6 \%\end{array}$ & $\begin{array}{c}6.1 \% \\
\pm 3.6 \% \mathrm{~B}\end{array}$ & $\begin{array}{c}P= \\
0.0021\end{array}$ \\
\hline $\begin{array}{r}\text { '100 } \mu \mathrm{M} \text { ' } \\
\text { Vehicle + IVC }\end{array}$ & $\begin{aligned} & 9.6 \% \\
\pm & 3.3 \% \mathrm{Ca}\end{aligned}$ & $\begin{array}{c}31.3 \% \\
\pm 7.0 \% \text { b }\end{array}$ & $\begin{array}{c}49.4 \% \\
\pm 4.5 \%{ }^{\mathrm{Cc}}\end{array}$ & $\begin{aligned} & 9.8 \% \\
& \pm 4.5 \% \mathrm{Ba} \\
&\end{aligned}$ & $\begin{array}{c}P= \\
0.0006\end{array}$ \\
\hline $\begin{array}{r}\text { '10 } \mu \mathrm{M} \text { ' } \\
\text { Vehicle + IVC }\end{array}$ & $\begin{array}{c}11.2 \% \\
\pm 2.5 \% \mathrm{Ca}\end{array}$ & $\begin{array}{r}25.8 \% \\
\pm 3.6 \% \text { b }\end{array}$ & $\begin{array}{c}53.1 \% \\
\pm 3.8 \% \mathrm{Cc}\end{array}$ & $\begin{aligned} & 9.9 \% \\
& \pm 3.8 \% \\
&\end{aligned}$ & $\begin{array}{c}P= \\
0.0001\end{array}$ \\
\hline$P$-value & $P<0.0001$ & $\begin{array}{c}P= \\
0.4119\end{array}$ & $\begin{array}{c}P< \\
0.0001\end{array}$ & $P<0.0003$ & \\
\hline
\end{tabular}

Table 3.2. Effect of proteasomal inhibition on zinc signature. Data are presented as mean \pm s.e.m. ( 3 biological replicates). Values with different uppercase superscripts $(A, B, C, D)$ indicate significant difference between the control (fresh, ejaculated spermatozoa and vehicle controls) and treatment groups and lowercase superscripts $(a, b, c)$ indicate significant difference between signatures as determined by Duncan's Multiple Range test ( $P$-value by treatment and signature in table). Both $\mathrm{PI}+$ and $\mathrm{PI}$ - cells were included in this analysis. Treatment column refers to proteasomal inhibitors MG132, Clasto-Lactacystin ß-Lactone (CLBL) and epoxomicin (Epox). A total of 10,000 cells were measured for each data point. 


\begin{tabular}{|r|c|c|c|}
\hline Boar & Farrowing Rate & Average Litter & Estrus Cycles \\
\hline Boar A & $90.5 \%$ & 10.03 & 42 \\
\hline Boar B & $89.5 \%$ & 9.52 & 38 \\
\hline Boar C & $65.2 \%$ & 7.40 & 23 \\
\hline Boar D & $57.1 \%$ & 7.17 & 21 \\
\hline
\end{tabular}

Table 3.3. Boar fertility trial records. Includes farrowing rate, average litter size, and number of estrus cycles (n).

\section{Movie Legends}

Movie 3.1. Comparison of sperm motility under varied IVC conditions. Left panel shows regular (as in not hyperactivated) sperm motility of non-IVC spermatozoa. Middle panel shows hyperactivated spermatozoa at 30 minutes after incubation under experimental IVC conditions. Right panel shows similar hyperactivated motility at 30 minutes after incubation in IVC medium containing $15 \mathrm{mM}$ sodium bicarbonate. Final DIC video output 30 frames per second. Available for download at: https://static-content.springer.com/esm/art\%3A10.1038\%2Fs41467-018-045 23-y/MediaObjects/41467 20184523 MOESM4 ESM.mp4

Movie 3.2. Hyperactivated spermatozoa have zinc signature 2. Video imaging of sperm zinc signature state $30-60$ minutes into IVC. On left, still image of fluorescence of zinc probe FZ3 (green; 2.0 seconds acquisition time) with video of spermatozoa DIC to right (DIC final video output 30 frames per second). Signature 1 spermatozoon displays normal, non-capacitating motility; signature 2 spermatozoon displays capacitation-induced, hyperactivated motility; signature 3 
spermatozoon, presumed to be post-capacitated, shows no motility. Available for download at: https://static-content.springer.com/esm/art\%3A10.1038\%2Fs41467$\underline{\text { 018-04523-y/MediaObjects/41467 } 20184523 \text { MOESM6 ESM.mp4 }}$

Movie 3.3. Zinc signature in motile vs. immotile spermatozoa after IVC. Hyperactivated signature 2 spermatozoon shows both the head and the tail midpiece labelling, the latter having a fanned appearance due to hyperactivated tail movement and long acquisition time required to record the fluorescent signal. Post-capacitated immotile signature 3 spermatozoon has labelling restricted to midpiece. FZ3 acquisition for 2.0 second and DIC final video output 30 frames per second. Available for download at: https://static-content.springer.com/esm/ art\%3A10.1038\%2Fs41467-018-04523-y/MediaObjects/41467 20184523 MOESM7 ESM.mp4

Movie 3.4. Zona-bound motile spermatozoa have zinc signature 2. On left, still image fluorescence of zinc probe FZ3 (green) and sperm head (blue) with MII oocyte acquired at the start of video recording ( 2.0 seconds acquisition time). Time lapse video recording of $\mathrm{Zn}$-induced fluorescence was not possible due long acquisition time and rapid photobleaching. On right, differential interference contrast (DIC) video of the zona-bound, hyperactivated, zinc signature 2 spermatozoon at seven o'clock position (DIC final video output 30 frames per second). Available for download at: https://static-content.springer.com/esm/art\%3 A10.1038\%2Fs41467-018-04523-y/MediaObjects/41467 20184523 MOESM5 ESM.mp4 


\section{CHAPTER 4}

\section{BOAR EJACULATE SEQUENCE ENDOWS SPERM ZINC SIGNATURE SUBPOPULATIONS AND CAPACITATION-INDUCED ZINC EFFLUX IS NECESSARY FOR OVIDUCTAL SPERM RESERVOIR GLYCAN RELEASE \& ACTIVATION OF ZONA PELLUCIDA PROTEINASE MMP2}

\section{Manuscript in preparation.}

\subsection{Abstract}

Building on our recent discovery of the zinc signature phenomenon present in boar, bull, and human spermatozoa, we have further characterized the role of zinc ions in the spermatozoa's pathway to fertilization in domestic boar, a species with a distinct fractioned pattern of ejaculation. The zinc signature differed between three major boar ejaculate fractions, the initial, the sperm-rich and the post-sperm rich fraction. These differences set in sperm ejaculatory sequence likely establish two major sperm cohorts; one destined for populating the sperm oviductal reservoir and the other that is capable of fertilizing mature, ovulated oocytes at the time of mating/insemination. The inclusion of $\mathrm{ZnCl}_{2}$ in semen extender prevented spontaneous, pathological capacitation, by day 3 of extended liquid semen storage and could allow for reducing the sperm number per artificial insemination (AI) dose to increase the usage of high genetic value sires. Further, newly formulated semen extender was able to mimic qualities of the pre-sperm rich fraction. On subcellular level, the capacitation induced $\mathrm{Zn}$-ion efflux allows for release from oviductal glycans studied with the oviductal epithelium mimicking glycan binding assay. Sperm zinc efflux also activates zinc-containing enzymes involved in sperm penetration of the zona pellucida, such as the inner acrosomal membrane 
metalloproteinase MMP2 that had a severely reduced activity in the presence of zinc ions by gel zymography. In context of the fertilization-induced oocyte zinc spark and the ensuing, oocyte-issued polyspermy-blocking zinc shield, the inhibitory effect of zinc on sperm-borne enzymes may contribute to the fast block of polyspermy. Altogether, our findings establish a new paradigm on the role of zinc ions in sperm function, and pave the way for the optimization of $\mathrm{Al}$ and semen distribution.

\subsection{Introduction}

It is widely understood that zinc ions $\left(\mathrm{Zn}^{2+}\right)$ play an important role in male fertility, starting with C. elegans (Chu, 2018) through higher order mammals (Colagar et al., 2009; Andreychenko et al., 2016; Nenkova et al., 2017) [for review see (Zhao et al., 2016; Fallah et al., 2018; Kerns et al., 2018b)], but its role in creating subpopulations of fertilization competent spermatozoa was not known until discovery of the zinc signature in boar, bull, and human spermatozoa (Kerns et al., 2018a). Although pre-requisite for fertility (Austin, 1951; Chang, 1951), sperm capacitation is a terminal maturation event leading to rapid cell death unless fertilization occurs (Jaiswal and Eisenbach, 2002). The zinc signature is tied directly to key sperm capacitation states: hyperactivation, acrosomal modification, acrosomal exocytosis, and the ability to detect/penetrate the oocyte zona pellucida [beginning, midpoint, late, and final capacitation states, (Kerns et al., 2018a)]. 
Artificial insemination (AI) is used by a vast amount of the U.S. swine industry $(95 \%+$ sows are mated by $\mathrm{Al})$, and is a valuable method to leverage valuable sire genetics and safeguard herd health. Besides the presence of antibiotics in boar semen, currently believed good standard operating procedures discard the first pre-sperm rich fraction due to high bacterial loads (Knox, 2007). However, literature suggests that spermatozoa in this initial fraction are the most fertile ones (Zhu et al., 2000), have reduced DNA fragmentation (Hebles et al., 2015), are more fit for cryopreservation survival (Rodriguez-Martinez et al., 2008), and are overrepresented in the sperm reservoir (Wallgren et al., 2010), thus earning its nickname, the "vanguard cohort" (Wallgren et al., 2010). This cohort is much more sensitive to $26 \mathrm{~S}$ proteasome inhibition of capacitation than the rich/post-rich fractions (Kerns et al., 2018a), indicating a previously unknown, distinct early capacitation event occurring at ejaculation and regulated by sperm-borne proteasomes. What distinguishes these vanguard spermatozoa as biologically different from the rest, remains unknown. Thus, boar semen extenders are not capable of capitalizing on the fertile aspects of this cohort. One of the most widely used boar semen extenders that serve as a basis for all modern-day extenders is the Beltsville Thaw Solution (BTS). The BTS was originally formulated in 1975 for use of thawing cryo-preserved semen (Pursel and Johnson, 1975) and updated for fresh semen in 1988 (Johnson et al., 1988).

Literature has recently alluded that BTS promotes premature sperm capacitation and might cause spermatozoa to advance through in vitro capacitation too quickly 
(Schmid et al., 2013); however, this has not been fully characterized, especially its relationship with the sperm zinc signature. Additionally, vanguard cohort-friendly semen supplements or extenders have not been developed, which could increase sperm capability to bind to the oviductal sperm reservoir, especially given sperm processing techniques that produce capacitation-like changes that are detrimental to sperm oviductal reservoir binding (Winters et al., 2018).

Finally, in the molecular/biochemical age, research has lost sight of Chang's original physiological definition of capacitation (Gervasi and Visconti, 2016): the acquisition of the capacity to fertilize (Chang, 1951). The ability to understand the biology allowing for this fertilization-enabling event could help preserve livestock semen in a biologically desired state, allowing spermatozoa to acquire fertilization competency only at the desired time. Similarly, understanding every aspect necessary to acquire this capacity can help us in both predicting fertility but also positively or negatively control male fertility, as specific applications desire. As we previously proposed, the zinc spark and resulting zinc shield might serve as a new anti-polyspermy defense mechanism (Kerns et al., 2018a), and while brain research has suggested the same MMP2 co-involved in zona pellucida penetration is inhibited by $\mathrm{Zn}^{2+}$ (Backstrom et al., 1992), it has not been confirmed whether sperm-borne MMP2 would be susceptible to decreased activity in the presence of $\mathrm{Zn}^{2+}$. 
(A comprehensive Materials and Methods is located on page 111.)

\subsection{Results}

Here, we show the boar sperm zinc signature is significantly different among ejaculate fractions, with most pre-sperm rich spermatozoa possessing the noncapacitated signature $1(93.5 \pm 1.4 \%)$ as opposed to the rest of the ejaculate possessing mostly signature 2 , associated with early stages of capacitation (rich, $91.5 \pm 1.1 \%$; post-rich, $87.5 \pm 1.8 \%$; whole ejaculate, $88.0 \pm 1.8 \%$; Fig. 4.1, summarized in Table 4.1; $P$-value $<0.0001)$. Further, addition of $1.0 \mathrm{mM} \mathrm{Zn^{2+ }}$ (from $\mathrm{ZnCl}_{2}$ ) to BTS prevented spontaneous, storage-induced protein tyrosine phosphorylation $(\mathrm{pY})$ detected by Western blot, a well-documented hallmark of sperm capacitation (Fig. 4.2). Addition of $\mathrm{ZnCl}_{2}$ to $\mathrm{BTS}$ did not allow spermatozoa to attain a zinc signature 1 state, thus the development of MU Vanguard extender. MU Vanguard extender allowed increased percentage of spermatozoa in a zinc signature 1 state compared to BTS after 24 hours of incubation (Fig. 4.3a \& c, summarized in Table 4.2; P-value < 0.0001). Further, MU Vanguard extender displayed significantly decreased percentage of spermatozoa with acrosomal modifications (shown by shift to IBFC gate PNA+, Fig. 4.3b \& d; $P$-value $<0.01$ ) and decreased percent spermatozoa with compromised plasma membranes (PI+,

Fig. 4.3e; $P$-value $<0.01 ; n=4$ biological replicates for all experiments; 10,000 spermatozoa analyzed per treatment). 
While the relative concentration of $\mathrm{Zn}^{2+}$ in seminal fluids is the highest of all bodily fluids [boar (Boursnell et al., 1972), human (Liu et al., 2009)], the concentration of $\mathrm{Zn}^{2+}$ decreases as the spermatozoa reach the site of fertilization to less than 15 $\mu \mathrm{M}$, a concentration less than that of blood serum (Patek and Hagenfeldt, 1974; Ménézo et al., 2011). We thus set out to examine if $\mathrm{Zn}^{2+}$ loaded (zinc signature 1) spermatozoa were capable of detachment from oviductal glycans in response to ovulatory signaling progesterone simulated by progesterone $\left(\mathrm{P}_{4}\right)$ infusion, and if the decreased $\mathrm{Zn}^{2+}$ concentration found in the oviduct was necessary for sperm release. Oviductal glycan assay showed that $0.5 \mathrm{mM}, 1.5 \mathrm{mM}$, and $2.5 \mathrm{mM} \mathrm{ZnCl}_{2}$, but not $15 \mu \mathrm{M} \mathrm{Zn}^{2+}$ or $2.5 \mathrm{mM} \mathrm{CaCl}_{2}$ (divalent cation negative control), prevented $P_{4}$-induced release from oviductal glycans (Fig. 4.4; $n=3$ biological replicates; $P$ value $<0.05)$.

Finally, we examined whether the fertilization-induced $\mathrm{Zn}$ oocyte spark and resulting $\mathrm{Zn}$ shield's inhibition may serve as a possible anti-polyspermy defense mechanism. While ZP proteinase acrosin has already been shown to be inhibited by $\mathrm{Zn}^{2+}$ (Steven et al., 1982), mouse knock out studies have shown acrosin may not be the sole ZP-targeting proteinase/zona lysin (Baba et al., 1994). It has been newly discovered that sperm Zn-metalloproteinase MMP2, located on the sperm inner acrosomal membrane (IAM), is an additional sperm-borne zona lysin (Ferrer et al., 2012a). To understand if $\mathrm{Zn}^{2+}$ blocks sperm-borne MMP2, we used zymography paired with $\mathrm{Zn}^{2+}$ blocking of boar and bull (as a positive control because the cited MMP2 work was performed with bull spermatozoa) sperm 
extracts. The presence of $1.5 \mathrm{mM} \mathrm{ZnCl} 2$ prevented gelatin breakdown of 72 and $92 \mathrm{kDa}$ proteins (MMP2 and 9 respectively) as well MMP2 and MMP9 expressing trophoblast cell line extract (positive control) while smaller sized proteins had increased gelatin breakdown in boar as compared to vehicle block (Fig. 4.5, $n=2$ biological replicates). Thus, we can confirm sperm-borne MMP2 activity is severely reduced in the presence of seminal fluids levels of $\mathrm{Zn}^{2+}$ while upregulating other proteins with gelatinase activity.

\subsection{Discussion}

A vast majority of sperm function studies rely strictly on a biochemical perspective, at times with little regard to the basic ejaculate physiology. Reflective of a widespread misunderstanding of seminal plasma is the belief that it is a relatively homogenous fluid, like that of blood plasma, a fluid with well-regulated homeostasis. Contrarily, seminal plasma is anything but this, thus referred to as seminal fluids by some (Bjorndahl and Kvist, 2003). The zinc signature differences uncovered here between the pre-sperm rich fraction and the rest of the ejaculate can likely be attributed to the composition of seminal fluids engulfing the respective fractions. The pre-rich fraction fluid originating from the prostate is known to have the largest amounts of free $\mathrm{Zn}^{2+}$ while later portions of the ejaculate containing mostly vesicular gland fluids are low in $\mathrm{Zn}^{2+}$, at least in humans (Huggins and Johnson, 1933). 
While boar seminal vesicular fluids contain $\mathrm{Zn}^{2+}$ (Lavon and Boursnel, 1975), most of this free $\mathrm{Zn}^{2+}$ is chelated by seminal vesicle citrate (Kvist et al., 1990) and may be bound to other $\mathrm{Zn}$-interacting seminal fluid proteins such as spermadhesin PSP-1 (Campanero-Rhodes et al., 2006). Additionally, progression of these later ejaculate fluids have an increase in $\mathrm{pH}$, which is regarded as necessary for sperm activation at ejaculation (Rodriguez-Martinez et al., 2009). Given spermatozoa from the pre-sperm rich fraction are more capable of binding the oviductal reservoir than the remainder of the ejaculate (Rodriguez-Martinez et al., 2009), combined with knowledge that only non-capacitated spermatozoa are capable of binding, it comes as no surprise that there is a difference in the capacitation-associated zinc signatures of these fractions. Together, these zinc signature differences, predetermined by ejaculatory sequence, might destine which spermatozoa bind the oviductal reservoir, quiescently awaiting later ovulated oocytes (the pre-sperm rich, zinc signature 1 spermatozoa), while the sperm-rich and post-sperm rich fraction spermatozoa that have undergone early stages of $\mathrm{Zn}$-signaling capacitation (Signature 2) might be destined only for immediate fertilizing of ovulated, fully mature oocytes upon time of mating/insemination. Alternatively, this later sperm cohort and seminal fluids could serve as a vehicle to stimulate uterine and oviductal epithelial cells' response to semen deposition, priming for imminent embryo implantation. The theory of seminal fluids eliciting a stimulatory response to prime for pregnancy (Robertson, 2005) is supported by meta-analysis of human publications. Meta-analysis confirmed an improvement in in vitro fertilization pregnancy rates when couples had sexual intercourse around the time of oocyte 
pick-up or embryo transfer (Crawford et al., 2015). Understanding the zinc signature state of the three sperm cohorts in relationship to time of insemination and ovulation might prove useful in creation of new semen extenders that are specifically formulated for inseminations prior to detectable ovulation.

It is known that $\mathrm{Zn}^{2+}$ serves as a de-capacitating factor by inhibiting hydrogen voltage channel HVCN1 (Lishko and Kirichok, 2010). Inhibiting HVCN1 would theoretically prevent early stage capacitation-associated intracellular $\mathrm{pH}$ rise and the CatSper channel dependent $\mathrm{Ca}^{2+}$ entry. The addition of $1.0 \mathrm{mM} \mathrm{ZnCl} 2$ to BTS eliminated storage-induced, capacitation-indicating pY by day three of incubation (Fig. 4.2b). While $1.0 \mathrm{mM} \mathrm{ZnCl} 2$ reduced $\mathrm{pY}$ in the present study, it did not allow spermatozoa to take up $\mathrm{Zn}^{2+}$ and achieve a signature 1 state and did not prevent acrosomal modification seen on day 3 of BTS stored semen. This could indicate that $\mathrm{pY}$ is not necessary for acrosomal modification; however, that is not the focus of this study and to verify this would require follow up studies using new single-cell Western blot analysis techniques (Kaelberer et al., 2018). Given this as well as the biochemical differences of the different fractions of the ejaculate and its effect on the zinc signature, we aspired to create an extender that mimicked the pre-sperm rich seminal fluid environment.

Our new MU Vanguard extender improves zinc management and allowed spermatozoa to remain in a non-early stage capacitation state (zinc signature 1; Fig. 4.3a \& c) during $24 \mathrm{~h}$ storage, similar to that of the pre-sperm rich fraction, 
and reduced the late stage, capacitation-induced acrosomal modification (Fig. 4.3b \& d) compared to BTS. Effectively, this treatment paused early stages of sperm capacitation. At current time, further buffer and energy management is needed to extend the lifespan of these spermatozoa past 24 hour as high agglutination is occurring around 36-48 hours (data not shown).

Assuming that the pre-sperm rich fraction spermatozoa have an increased ability to bind the oviductal sperm reservoir (Wallgren et al., 2010) and that capacitation facilitates sperm release, we wanted to understand if $\mathrm{Zn}^{2+}$ would prevent spermatozoa release from oviductal glycans. Given the role of $\mathrm{Zn}^{2+}$ in inhibiting HVCN1, it comes as no surprise that spermatozoa were incapable of release from oviductal glycans in the presence of high $\mathrm{Zn}^{2+}$, even after the sperm release cue given by progesterone addition. This further supports the sperm release from the oviductal reservoir being a sperm capacitation-driven event in response to female reproductive tract ovulatory cues, rather than a female-oviductal glycan degradation-related release, as discussed by others (Suarez, 2008). Zinc may also inhibit or reduce the activity of the $26 \mathrm{~S}$ proteasome, whose activity has been shown as necessary for release from oviductal glycans (Sharif et al., 2017). Zinc has been implicated in inhibiting 26S proteasome-dependent proteolysis in HeLa cells (Kim et al., 2004) and the 20 S proteasomal core activity in bovine brain tissue (Amici et al., 2002); however, it remains unknown if $\mathrm{Zn}^{2+}$ would inhibit or reduce sperm $26 \mathrm{~S}$ proteasome-dependent proteolysis. 
In order for acrosomal exocytosis and activation of ZP proteinases to occur, a spermatozoon must first undergo acrosomal modification. Not shown here due to difficulty to capture, it was observed that spermatozoa transitioning from signature 2 (hyperactivated) to signature 3 (modified acrosome) and final cell death have a very limited lifespan (ranging from 30 seconds to 2 minutes). This short life span after [perceivably] full acquisition to fertilize comes as no surprise, as Aitken et al. (2015) have suggested reactive oxygen species (ROS) creation critical to capacitation success is yet a risky strategy for spermatozoa due to their lack of antioxidant protection. If true, herein lies the origin of current under-appreciation of sperm ion fluxes within sperm fertilization biology.

The major role of zinc in the sustenance of zinc-containing proteins can be catalytic, cocatalytic, or structural (McCall et al., 2000). Zinc is a required ion for many MMP activities, including MMP2 and MMP9. Both MMPs have two $\mathrm{Zn}^{2+}$ sites and four (MMP2) and three (MMP9) $\mathrm{Ca}^{2+}$ sites (UniProt Acc no.: P08253 and P14780) (UniProt Consortium, 2018). A finely tuned balance in zinc:calcium ratio is therefore important for MMP activity (Vettakkorumakankav and Ananthanarayanan, 1999). At a high concentration, $\mathrm{Zn}^{2+}$ competitively binds the $\mathrm{Ca}^{2+}$ site, thereby causing a structural change and inhibiting proteinase activity of MMPs (Backstrom et al., 1992). Conversely, when $\mathrm{Zn}^{2+}$ ion is chelated, MMP activity is inhibited (Lippa, 1991). Environmental exposure to $\mathrm{Cd}^{2+}$ in rats has shown decreases in both MMP2 and MMP9 testicular tissue activities (Lacorte et al., 2015), likely by competitive binding to $\mathrm{Zn}^{2+}$ and/or $\mathrm{Ca}^{2+}$ sites. Interestingly, low 
molecular weight sperm proteinases were upregulated in our $\mathrm{Zn}^{2+}$ block (Fig. 4.5b) and deserve future exploration. Thus, as we originally proposed that the oocyteissued zinc shield may serve as an anti-polyspermy defense mechanism, (Kerns et al., 2018a) we herein prove the precise mechanism - inhibition of a major ZP proteinase MMP2. Given that $\mathrm{Zn}^{2+}$ repels spermatozoa that are susceptible to $\mathrm{P}_{4}$ chemotaxis (Guidobaldi et al., 2017), this pathway may offer a new, non-hormonal contraceptive target. This target could be designed for the male or female, as an abundance of $\mathrm{Zn}^{2+}$, compounds that competitively target catalytic $\mathrm{Zn}^{2+}$ sites, or chelation of $\mathrm{Zn}^{2+}$. Chelation of MMP2 bound $\mathrm{Zn}^{2+}$ by application of topical carbonic anhydrase inhibitors has served as a biological exploitation in lowering intraocular pressure in glaucoma patients (Huang et al., 2011).

Here, we show that the differences in the zinc signature based on ejaculatory sequence likely predestine the fertilizing cohort of spermatozoa, those that populate the oviductal reservoir (pre-sperm rich) and those that have already undergone early stages of sperm capacitation (rich and post-rich fractions). Additionally, we show that in the presence of relative high $\mathrm{Zn}^{2+}$, spermatozoa are incapable of release from oviductal glycans and activation sperm proteinase MMP2, likely necessitating the $\mathrm{Zn}^{2+}$ efflux observed during sperm capacitation. Others have previously shown pre-rich fraction spermatozoa to be more fertile than the rest of the ejaculate; however, mechanisms to emulate this vanguard group have been lacking. Here we show that managing the sperm zinc signature to mimic that of the pre-sperm rich spermatozoa decreases spontaneous, storage-induced sperm capacitation. Our results imply that Al extenders should be re-formulated, 
not simply to mix semen with an energy and electrolyte-buffered cocktail, but to best mimic sperm ejaculatory physiology in perspective to time on their pathway to fertilization. This optimization likely will prove useful in the swine industry's quest for fewer spermatozoa per dose.

\subsection{Acknowledgments}

Supported by the National Institute of Food and Agriculture (NIFA), U.S. Department of Agriculture (USDA) grant number 2015-67015-23231 (P.S.), USDA NIFA Graduate Fellowship award number 2017-67011-26023 (K.K.), grant number 5 R01 HD084353-02 from NIH National Institute of Child and Human Development (P.S.), NSERC (RGPIN/192093) (RO), and seed funding from the Food for the 21st Century Program of the University of Missouri (P.S.). We thank staff of the National Swine Research and Resource Center, University of Missouri, as well as

Dr. Randall Prather and his associates for wild-type boar semen collection, funded by National Institutes of Health (NIH) U42 OD011140. Author thanks Momal Sharif and Dr. David Miller for performing the oviductal glycan study as well as Wei Xu, Lauren Hamilton, and Dr. Richard Oko for performing the zymography study. 


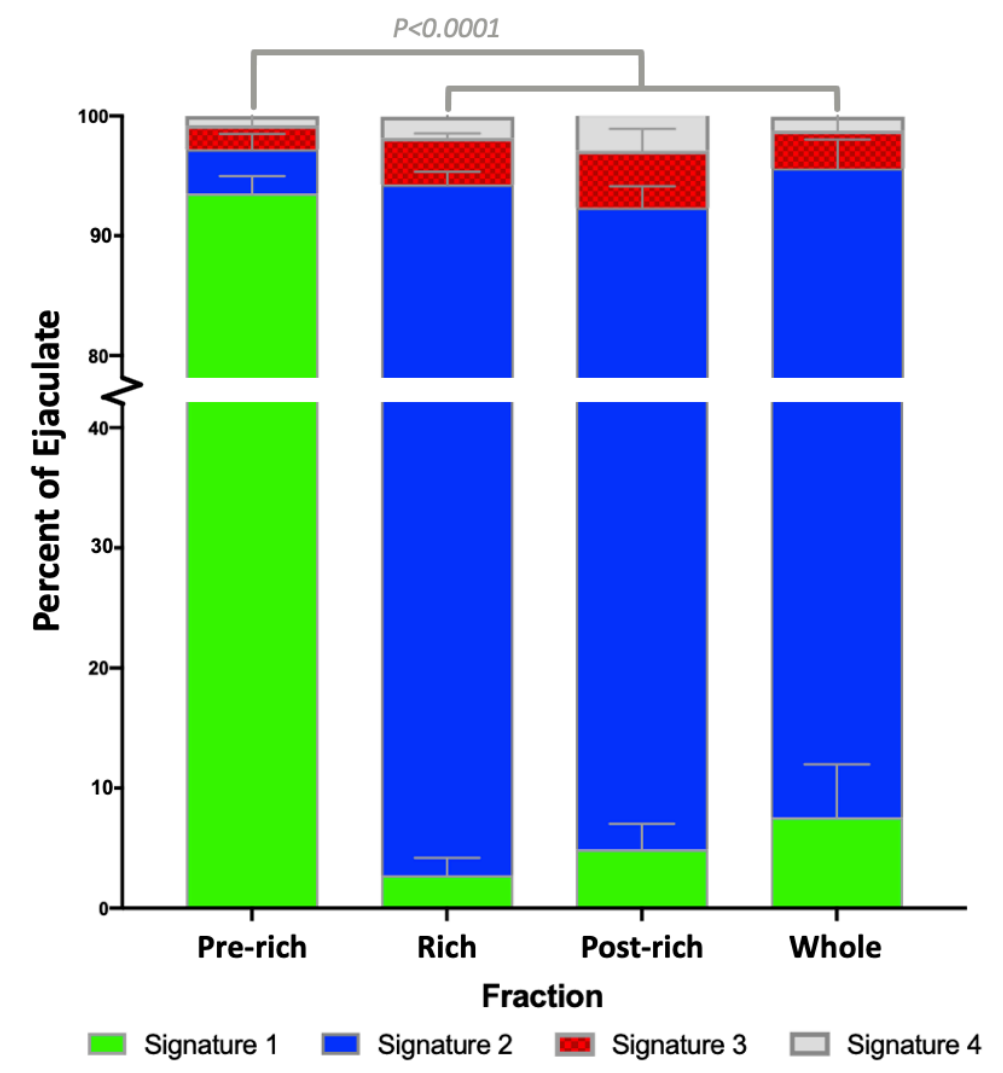

Figure 4.1. Boar sperm zinc signature differs by ejaculate fraction. Quantitative analysis of the sperm zinc signature across the three ejaculate fractions determined that the pre-sperm rich fraction was significantly different $(P$-value $<$ 0.0001) from the sperm-rich \& post-sperm rich fractions as well as when mixed as a whole ejaculate. Three biological replicates $(n=3)$ were analyzed with a total of 10,000 cells measured for each treatment. 


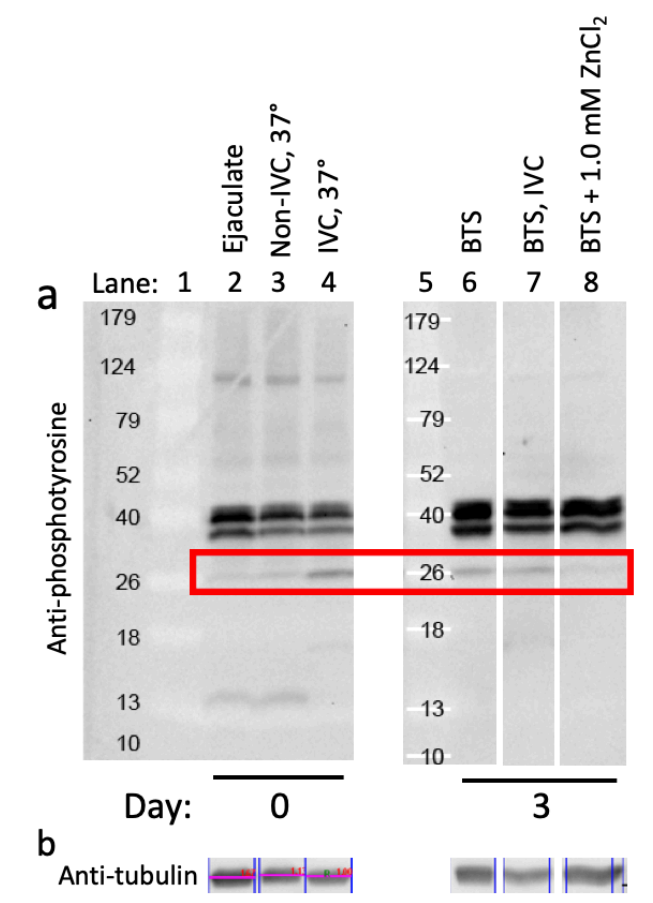

Figure 4.2. $\mathrm{BTS}+1.0 \mathrm{mM} \mathrm{ZnCl} 2$ prevents spontaneous sperm tyrosine phosphorylation ( $\mathrm{pY}$ ), an indicator of sperm capacitation. a, Anti-phosphotyrosine Western blot of sperm extracts from treatments in the following lanes on Day 0:1) marker; 2) non-IVC, ejaculated; 3) non-IVC, $37^{\circ}$ incubation $4 \mathrm{~h}$; 4) IVC, $37^{\circ}$ incubation 4 h; 5) marker; Day 3: 6) BTS extended semen; 7) BTS, IVC, $37^{\circ}$ incubation $4 \mathrm{~h}$; 8) BTS $+1.0 \mathrm{mM} \mathrm{Zn} \mathrm{extended} \mathrm{semen.} \mathrm{Under} \mathrm{IVC} \mathrm{conditions,} \mathrm{a}$ band in the $26 \mathrm{kDa}$ region increased protein tyrosine phosphorylation, a traditional hallmark of sperm capacitation, compared to ejaculate and non-IVC sperm extracts. On Day 3, there is no difference in this band between sperm extracts stored in BTS compared to 4 hours IVC, while semen incubation in BTS with 1.0 $\mathrm{mM} \mathrm{ZnCl} 2$ prevented spontaneous, storage-induced $\mathrm{pY}$. b, Anti-tubulin Western blots displayed no relative difference in protein load. Representative of 2 biological replicates is shown. 

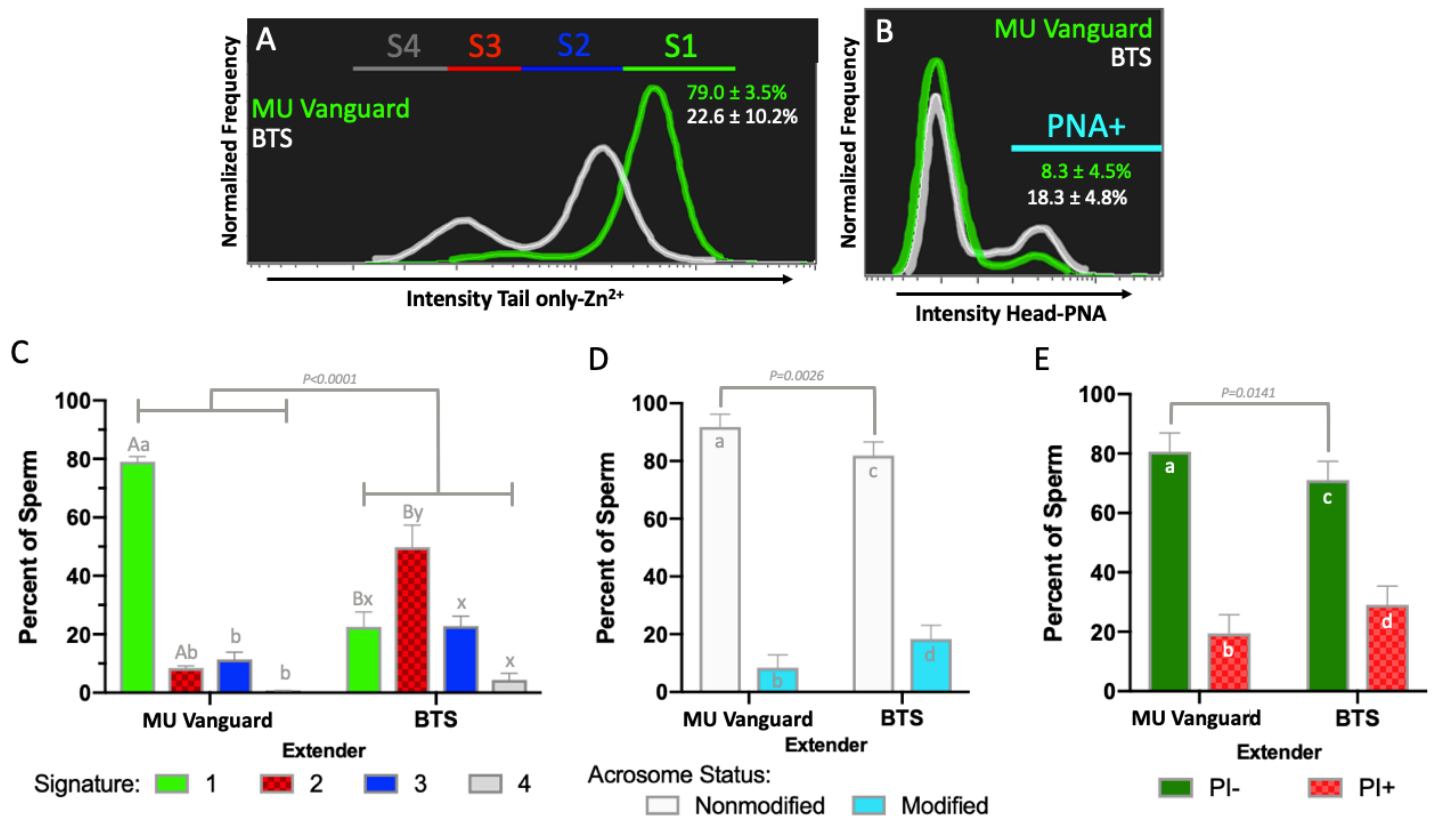

Figure 4.3. MU Vanguard extender prevents storage-induced sperm capacitation. A. Typical IBFC zinc signature histograms of boar spermatozoa stored for 24 hours in MU Vanguard (green) and BTS (white) with the average \pm SD percent of spermatozoa with signature 1. B. Typical IBFC acrosome status histogram of boar spermatozoa stored for 24 hours in MU Vanguard (green) and BTS (white) with the average \pm SD percent of spermatozoa with modified acrosomes. C. Quantitative analysis of the sperm zinc signature after $24 \mathrm{~h}$ incubation in the respective extenders; different uppercase superscripts $(A, B)$ indicate significant difference across extenders within signature $(P$-value $<0.01)$; different lowercase letters $(a, b$ \& $x, y)$ indicate significant differences between signatures within extender $(P$-value $<0.01)$. D. Quantitative analysis of the sperm acrosome status after $24 \mathrm{~h}$ of incubation in the respective extenders; different lowercase superscripts $(a, b, c, d)$ indicate significant differences in acrosome status $(P$-value $<0.01)$. E. Quantitative analysis of the sperm membrane integrity after $24 \mathrm{~h}$ of incubation in the respective extenders; different lowercase superscripts $(a, b, c, d)$ 
indicate significant differences in acrosome status $(P$-value $<0.01)$. Four biological replicates $(n=4)$ were analyzed with a total of 10,000 cells measured for each treatment. Numerical values shown in Table 4.2.

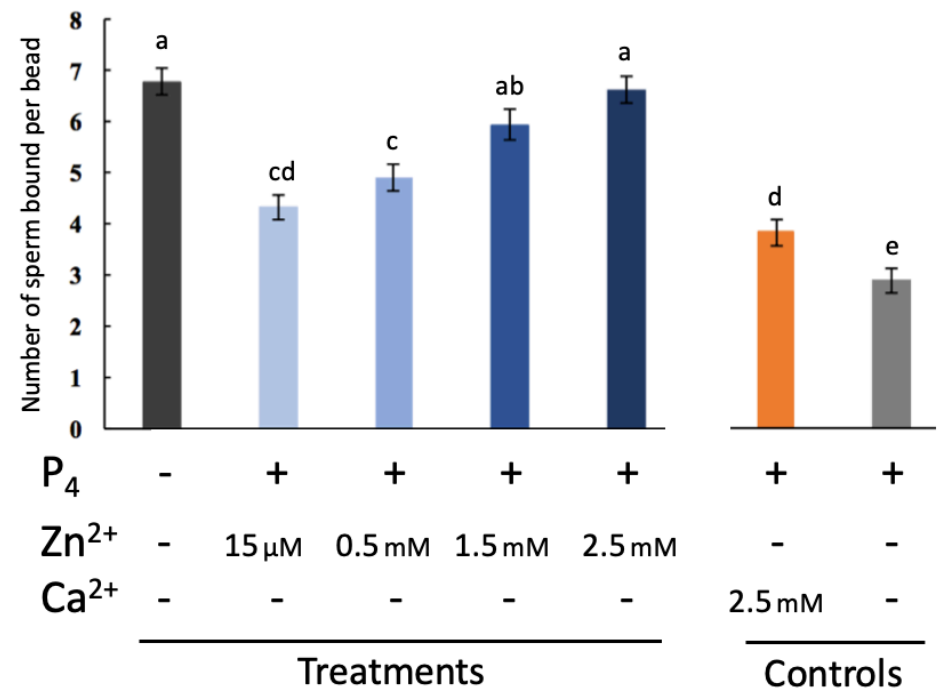

Figure 4.4. Zinc inhibits $P_{4}$-induced sperm release from oviductal glycans. Quantitative analysis revealed that $\mathrm{Zn}^{2+}$ inhibits $\mathrm{P}_{4}$-induced sperm release from oviductal glycans in a dose-dependent manner, with $2.5 \mathrm{mM} \mathrm{ZnCl} 2$ significantly different from $2.5 \mathrm{mM} \mathrm{Ca}^{2+}$ control. Different lowercase letters $(a, b, c, d, e)$ indicate significant difference across treatments $(P$-value $<0.05)$. Three biological replicates $(n=3)$ were analyzed. 


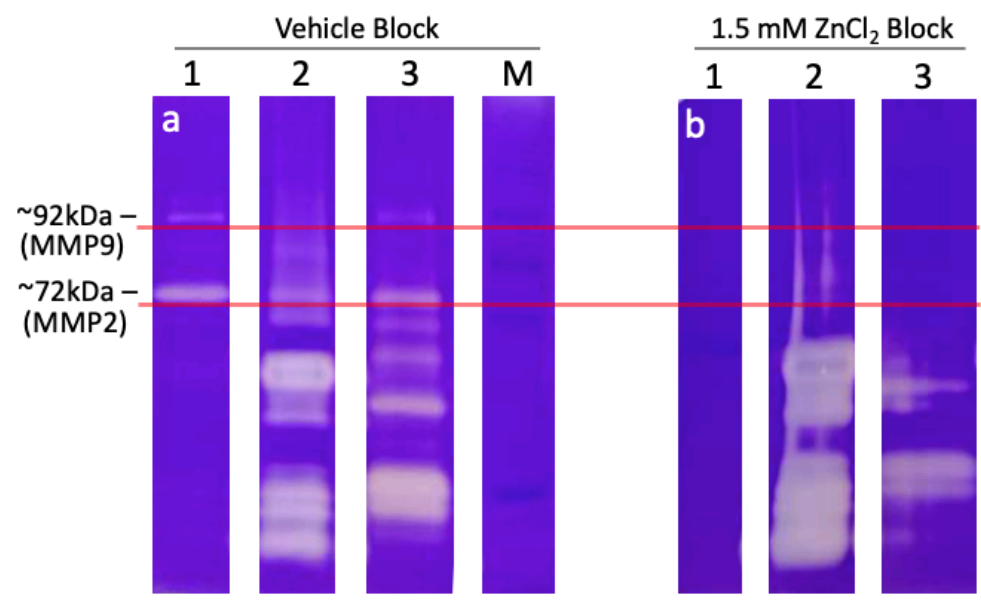

Figure 4.5. $\mathrm{Zn}$ inhibits the activity of sperm-borne ZP proteinase MMP2, a candidate zona lysin. Clear bands in the zymogram indicated enzymatic digestion of gelatin. A. Both boar and bull sperm extracts show enzymatic digestion at 72 and $92 \mathrm{kDa}$ in the vehicle block, that of MMP2 and MMP9 activities, respectively. B. The same bands showed a decreased enzymatic gelatin digestion when blocked with $1.5 \mathrm{mM} \mathrm{ZnCl} 2$ while up regulating the activity (increased enzymatic digestion) of the lower mass proteinases. Validation of this technique for sperm MMP activity, including MMP inhibitor GM6001, is covered in (Ferrer et al., 2012a). Red lines underline bands expected to be affected by treatment. Results typical of two biological replicates $(n=2)$ are shown. Lanes: 1) trophoblast cell line expressing MMP2 and MMP9 (positive control); 2) boar sperm extract; 3) bull sperm extract; M) marker. 


\begin{tabular}{|r|c|c|c|c|}
\hline & Signature 1 & Signature 2 & Signature 3 & Signature 4 \\
\hline Pre & $93.5 \pm 1.4 \%^{\mathrm{Aa}}$ & $3.7 \pm 1.3 \%^{\mathrm{Ab}}$ & $2.0 \pm 0.8 \%^{\mathrm{b}}$ & $0.8 \pm 0.4 \%^{\mathrm{b}}$ \\
\hline Rich & $2.8 \pm 1.5 \%^{\mathrm{Ba}}$ & $91.5 \pm 1.1 \%^{\mathrm{Bb}}$ & $3.9 \pm 0.4 \%^{\mathrm{a}}$ & $1.8 \pm 0.5 \%^{\mathrm{a}}$ \\
\hline Post & $4.9 \pm 2.1 \%^{\mathrm{Ba}}$ & $87.5 \pm 1.8 \%^{\mathrm{Bb}}$ & $4.8 \pm 1.8 \%^{\mathrm{C}}$ & $2.9 \pm 0.6 \%^{\mathrm{c}}$ \\
\hline Whole & $7.6 \pm 4.4 \%^{\mathrm{Ba}}$ & $88.0 \pm 2.4 \%^{\mathrm{Bb}}$ & $3.2 \pm 1.5 \%^{\mathrm{c}}$ & $1.2 \pm 0.6 \%^{\mathrm{w}}$ \\
\hline
\end{tabular}

Table 4.1. Boar ejaculate fraction endows sperm zinc signature subpopulations. Data are presented as mean \pm SEM. Values with different uppercase superscripts $(A, B)$ indicate significant difference by fraction $(P$-value $=0.0001)$ and lowercase superscripts $(a, b, c)$ indicate significant difference of zinc signatures (P-value < $0.0001)$. Three biological replicates $(n=3)$ were analyzed with a total of 10,000 cells analyzed per sample.

\begin{tabular}{|c|c|c|c|c|}
\hline & Signature 1 & Signature 2 & Signature 3 & Signature 4 \\
\hline BTS & $22.6 \pm 5.1 \%{ }^{\mathrm{Aa}}$ & $49.8 \pm 7.6 \%{ }^{\mathrm{Ab}}$ & $22.8 \pm 3.4 \%^{b}$ & $4.3 \pm 2.3 \%^{\mathrm{b}}$ \\
\hline MU Vanguard & $79.0 \pm 1.8 \%^{\mathrm{Ba}}$ & $8.5 \pm 0.6 \%^{\mathrm{Bb}}$ & $11.4 \pm 2.5 \%^{a}$ & $0.6 \pm 0.1 \%^{a}$ \\
\hline & PNA+ & & $\mathbf{P I +}$ & \\
\hline BTS & $18.3 \pm 2.4 \%^{\mathrm{A}}$ & & $29.0 \pm 3.2 \%{ }^{A}$ & \\
\hline MU Vanguard & $8.3 \pm 2.2 \%^{B}$ & & $19.4 \pm 3.1 \%^{B}$ & \\
\hline
\end{tabular}

Table 4.2. MU Vanguard extender prevents storage-induced sperm capacitation. Data are presented as mean \pm SEM. Values with different uppercase superscripts $(A, B)$ indicate significant difference by extender $(P$-value $\leq 0.01)$ and lowercase superscripts $(a, b)$ indicate significant difference of zinc signature (P-value < $0.0001)$. Four biological replicates $(n=4)$ were analyzed with a total of 10,000 cells measured for each treatment. 


\section{MATERIALS AND METHODS FOR ALL CHAPTERS}

\section{Reagents}

All reagents unless otherwise noted were from Sigma. FluoZin ${ }^{\mathrm{TM}}-3$, AM (FZ3; zinc probe) from ThermoFisher (F24195) was reconstituted with DMSO to a stock solution of $500 \mu \mathrm{M}$. Lectin PNA (Arachis hypogea/peanut agglutinin) conjugated to Alexa Fluor $^{\text {TM }} 647$ (PNA-AF647) from Invitrogen ${ }^{\text {TM }}$ (L32460). Fluo-4 NW (calcium probe) from ThermoFisher (F36206) was reconstituted using kit provided assay buffer. Hoechst 33342 (H33342) from Calbiochem (382065) was reconstituted with $\mathrm{H}_{2} \mathrm{O}$ to a stock solution of $18 \mathrm{mM}$. Propidium lodide $(\mathrm{PI})$ from Acros Organics (AC440300010) was reconstituted with $\mathrm{H}_{2} \mathrm{O}$ to a stock solution of $1 \mathrm{mg} \mathrm{mL}^{-1}$. Proteasomal inhibitors were from Enzo Life Sciences: MG132 (BML-PI102) was reconstituted with DMSO to a stock solution of $20 \mathrm{mM}$; Epoxomicin (Epox, BMLPI127) was reconstituted to a stock solution of $20 \mathrm{mM}$ (using MG132 stock); and clasto-Lactacystin $\beta$-Lactone (CLBL, BML-PI108) was reconstituted with DMSO to

a stock solution of $5 \mathrm{mM}$. Zn-chelator TPEN from Tocris (16858-02-9) was resuspended with 1:100 EtOH: $\mathrm{H}_{2} \mathrm{O}$ to a stock solution of $1 \mathrm{mM}$. Bovine serum albumin (BSA) was from Sigma (A4503). Anti-phosphotyrosine antibody, clone 4G10® was from EMD Millipore (05-321).

\section{Models, Semen Collection, and Processing}

Domestic boar (Sus scrofa) semen collection was performed under the guidance of approved Animal Care and Use (ACUC) protocols of the University of MissouriColumbia and purchased from the National Swine Research and Resource Center 
(University of Missouri, Columbia, MO). Boar semen for the fertility trial was collected, extended, and shipped by overnight parcel from a private boar stud following their established standard operating procedures and was not blinded. Boar collection was performed using the standard two gloved hand technique (Yi et al., 2012c). Only ejaculates with greater than $80 \%$ motility were used and no randomization was necessary as only one boar was studied at a time. The sperm rich fraction of boar ejaculate was used, except all IVC proteasomal inhibition studies used the pre-sperm rich fraction, which had increased viability and sensitivity to $26 \mathrm{~S}$ inhibition. Semen was immediately extended, within $2^{\circ} \mathrm{C}$, five times in Beltsville thawing solution (BTS) semen extender. Sperm concentration was then determined using a hemocytometer.

All semen washes were performed with a swing hinge rotor centrifuge at $110 \times \mathrm{g}$ for 5 minutes. Number of washes and g-force used were minimized as these were found to compromise results. Frozen-thawed bull (Bos taurus) spermatozoa were processed similarly as boar spermatozoa after being thawed for 45 seconds in $35^{\circ} \mathrm{C}$ water bath. For human spermatozoa, sperm donors signed informed consent and the samples were coded as to make the donors unidentifiable to researchers. All human sperm samples were handled and processed strictly as stipulated by an approved Internal Review Board (MU IRB) protocol. Donors were recruited by placing an advertisement for new fathers in the university mass e-mail newsletter. All semen was collected onsite at the Missouri Center for Reproductive 
Medicine and Fertility Clinic. Samples were then transported to the laboratory for analysis.

\section{Collection and Processing of Spermatozoa for Zinc Signature Fraction Analysis}

For each replicate, semen was collected from 3 to 5 mature Sus scrofa boars and analyzed immediately. Semen was washed in mTL-HEPES (composition described under following in vitro capacitation section) within 30 minutes after collection. Only samples with greater than $80 \%$ sperm motility were used for experiments. The pre-sperm rich fraction is traditionally discarded in industry because of a perceived increased bacterial load and to eliminate urine contamination, with the exception of automated collection system which collect the entire ejaculate. Thus, the first $2-5 \mathrm{~mL}$ of the pre-sperm rich fraction was not collected to help reduce chances of urine contamination, which otherwise killed spermatozoa and consequently reduced the zinc signature (data not shown). If any fraction was contaminated with urine, the entire replicate was discarded from data analysis. The pre-sperm rich fraction was identified as the first clear fluids, preceding the milkier sperm-rich fraction, and was 5-12 $\mathrm{mL}$. The sperm-rich fraction ranged in $35-50 \mathrm{~mL}$, and the post-rich fraction, which was less milky but whiter than the pre-sperm rich fraction, ranged from $70-110 \mathrm{~mL}$. 


\section{In Vitro Capacitation}

Fresh boar spermatozoa were capacitated using a protocol that rendered them capable of recognizing and binding to $\mathrm{ZP}$, as well as undergoing acrosomal exocytosis and penetrating the oocyte ZP (Zimmerman et al., 2011). IVC-induced changes to the acrosome and plasma membrane are shown in Fig. 3.2, including protein tyrosine phosphorylation changes. Briefly, spermatozoa were washed of seminal plasma once with noncapacitating media (NCM), a modified TL-HEPES medium, free of calcium dichloride $\left(\mathrm{CaCl}_{2}\right)$ and addition of $11 \mathrm{mM} \mathrm{D-glucose,} \mathrm{with}$ $\mathrm{pH}$ adjusted to 7.2. Spermatozoa were then resuspended in $0.5 \mathrm{~mL}$ in vitro capacitation (IVC) media, TL-HEPES-PVA supplemented with $5 \mathrm{mM}$ sodium pyruvate, $11 \mathrm{mM}$ D-glucose, $2 \mathrm{mM} \mathrm{CaCl} 2,2 \mathrm{mM}$ sodium bicarbonate, and $2 \%(\mathrm{~m} / \mathrm{v})$ bovine serum albumin, and incubated in a $37^{\circ} \mathrm{C}$ water bath for 4 hours, with sperm rotation performed every 60 minutes. Control incubations under non-IVC conditions used NCM. Proteasome inhibitors (100 $\mu \mathrm{M}$ MG132 and $10 \mu \mathrm{M}$ Epox/CLBL/MG132) were mixed with IVC media prior to sperm pellet resuspension. $100 \mu \mathrm{M} \mathrm{MG132}$ and '100 $\mu \mathrm{M}$ ' vehicle contained 0.5\% (v/v) DMSO. $10 \mu \mathrm{M}$ Epox/CLBL/MG132 and '10 $\mu \mathrm{M}$ ' vehicle contained 0.3\% (v/v) DMSO. PVA helped to decrease sperm aggregation and spermatozoa were pipetted repeatedly to dissociate sperm aggregates in a satisfactory manner prior to IBFC data acquisition. To confirm normal capacitation in our experimental IVC media, compared to $15 \mathrm{mM}$ sodium bicarbonate IVC media, we refer readers to Movie 3.1 (hyperactivation status; link). Both media support hyperactivated motility. Unlike murine or rodent sperm tyrosine phosphorylation, porcine tyrosine phosphorylation 
is much more modest, with less prominent changes during the course of capacitation. Therefore new bands after capacitation appear only at the molar weights of $32 \mathrm{kDa}$ (acrosin binding protein), and $21 \mathrm{kDa}$ protein (phospholipid hydroperoxide glutathione peroxidase; Fig. 3.2 e-f). Our results are in accordance with previous studies (Flesch et al., 1999; Tardif et al., 2001; Dube et al., 2003). Final acrosome and plasma membrane modification status is similar at end of IVC regardless of the two IVC treatment conditions; however, the rate of change and cell death is faster in $15 \mathrm{mM}$ sodium bicarbonate containing medium than experimental IVC medium (Fig. 3.2a-d). Altogether, this supports the use of experimental IVC medium over $15 \mathrm{mM}$ sodium bicarbonate containing medium to display the prolonged lifespan of spermatozoa as seen in in vivo capacitation.

\section{TPEN Zinc Chelation}

$\mathrm{Zn}^{2+}$ chelation was performed using TPEN (membrane permeable). $10 \mu \mathrm{M}$ TPEN was incubated with 40 million sperm per $\mathrm{mL}$ for 1 hour. Stock TPEN: $1 \mathrm{mM}$ in 1:100 EtOH:H2O.

\section{Multiplex Fluorescence Probing}

Upon 4 hours of IVC, sample size of $100 \mathrm{uL}$ (4 million spermatozoa) were incubated 30 minutes with 1:200 H33342, 1:200 PI, and 1:100 FZ3 for epifluorescence microscopy. Lower probe concentrations were necessary for IBFC due to camera detection differences, thus 1:1000, 1:1000, and 1:500 were used, respectively, with inclusion of 1:1000 PNA-AF647. For Fluo-4 calcium probe, we 
followed manufacturer protocol using identical cell concentrations. Spermatozoa were then washed of probes once and resuspended in corresponding IVC treatment media to allow complete de-esterfication of intracellular AM esters, as suggested by ThermoFisher's FZ3 protocol, followed by an additional wash and resuspended in $100 \mathrm{uL}$ PBS for IBFC analysis (or added to a slide for epifluorescence microscopy imaging).

\section{Epifluorescence Microscopy Imaging}

Live spermatozoa were imaged using a Nikon Eclipse 800 microscope (Nikon Instruments Inc.) with Cool Snap camera (Roper Scientific, Tuscon, AZ, USA) and MetaMorph software (Universal Imaging Corp., Downington, PA, USA). Images were adjusted for contrast and brightness in Adobe Photoshop CS5 (Adobe Systems, Mountain View, CA) to match the fluorescence intensities viewed through the microscope eyepieces.

\section{Super Resolution Microscopy Imaging}

Live spermatozoa were imaged using a Leica TCP SP8 STED (Lecia Microsystems, Wetzlar, Germany) and LAS X Life Science software (Lecia Microsystems, Wetzlar, Germany). 


\section{Image-based Flow Cytometric Data Acquisition}

IBFC data acquisition was performed following previous methodology (Kennedy et al., 2014). Specifically, using a FlowSight flow cytometer (FS) fitted with a 20x microscope objective (numerical aperture of 0.9 ) with an imaging rate up to 2000 events per sec. The sheath fluid was PBS (without $\mathrm{Ca}^{2+}$ or $\mathrm{Mg}^{2+}$ ). The flow-core diameter and speed was $10 \mu \mathrm{m}$ and $66 \mathrm{~mm}$ per sec, respectively. Raw image data were acquired using INSPIRE® software. To produce the highest resolution, the camera setting was at $1.0 \mu \mathrm{m}$ per pixel of the charged-coupled device. In INSPIRE ® FS data acquisition software, two brightfield channels were collected (channels

1 \& 9), one FZ3 image (channel 2), one PI image (channel 5), one side scatter (SSC; channel 6), one H33342 (channel 7), and one PNA-AF647 image (channel 11), with a minimum of 10,000 spermatozoa collected. The following lasers and power settings were used: $405 \mathrm{~nm}$ (to excite H33342): $10 \mathrm{~mW}$; $488 \mathrm{~nm}$ (to excite FZ3): $60 \mathrm{~mW}$; $561 \mathrm{~nm}$ (to excite Pl): $40 \mathrm{~mW}, 642 \mathrm{~nm}$ (to excite PNA-AF647): 25 $\mathrm{mW}$; and $785 \mathrm{nM} \mathrm{SSC} \mathrm{laser:} 10 \mathrm{~mW}$.

\section{IBFC Data Analysis}

Data were analyzed using IDEAS $\AA$ analysis software from AMNIS EMD Millipore. Gating approach used standard focus and single cell gating calculations created by IDEAS software (Fig. 3.1a \& b). To further clean up data for analysis, Feature Finder function was used to discover image-based calculations to discard spermatozoa laterally aligned with the camera, as opposed to anteriorly/posteriorly aligned, (Fig. 3.1c). Traditional flow cytometric analysis methods do not allow 
distinction of signature 1 and 2 based on whole cell FZ3 intensity, therefore creating a mask that only analyzes the sperm tail proved to be key in distinguishing these two populations. Such mask was created by taking a morphology mask of the brightfield (Fig. 3.1d), subtracting a 4-pixel dilation of H33342 (Fig. 3.1e), resulting in a mask to analyze fluorescence in the tail region only (Fig. 3.1f \& g). Mask dilation of $\mathrm{H} 33342$ fluorescence was necessary because $\mathrm{H} 33342$ labeling of the sperm nucleus did not cover the entirety of the sperm head, where FZ3 signal was high. This combined gating and masking strategy provided robust clean data, ready for signature analysis by plotting FZ3 intensity of this masked region, which is impossible with traditional flow cytometry. Gating boundaries for signatures 1, 2, and 3 were determined by the population segregations of fresh/ejaculate and IVC + vehicle treatments with signature status confirmed in the image gallery. Gating boundaries between signatures 3 and 4 were less evident in histograms and placed where spermatozoa lost FZ3 signal as determined using the image gallery. 


\section{Western Blotting (WB)}

Sperm pellets (15 million spermatozoa per pellet) were mixed with reducing SDSPAGE loading buffer, boiled for $5 \mathrm{~min}$ and briefly spun at $5000 \mathrm{~g}$. The SDS-PAGE was carried out on a 4-20\% gradient gels (PAGEr Precast gels; Lonza Rockland, Rockland, ME, USA) as previously described (Miles et al., 2013). The molecular masses of the separated proteins were estimated by using prestained Prosieve protein colored markers (Lonza Rockland) run in parallel. After SDS-PAGE, proteins were electro-transferred onto a PVDF Immobilon Transfer Membrane (Millipore, Bedford, MA, USA) using an Owl wet transfer system (Fisher Scientific) at a constant $50 \mathrm{~V}$ for $4 \mathrm{~h}$ for immunodetection (Miles et al., 2013).

\section{Sperm Oviductal Glycan Binding Assay}

Glycan-coated streptavidin-Sepharose High-Performance beads (GE Healthcare Bio-Sciences, Pittsburgh, PA, an average diameter of $34 \mu \mathrm{m}$ ) were used to test the ability of spermatozoa to detach from oviduct glycans (bi-SiaLN: biantennary 6-sialylated lactosamine oligosaccharide, suLe ${ }^{\mathrm{X}}$ : 3-O-sulfated Lewis $\mathrm{X}$ trisaccharide), in the presence of zinc and current believed sperm-release cue progesterone (Brssow et al., 2008). To link the glycans to beads, approximately 60 $\mu \mathrm{g}$ of each glycan (Bovin et al. 1993) covalently attached to a biotinylated polyacrylamide core were incubated with $20 \mu \mathrm{L}$ of streptavidin-Sepharose beads for $90 \mathrm{~min}$ at room temperature. Each $30-\mathrm{kDa}$ molecule of polyacrylamide had $20 \%$ glycan and $5 \%$ biotin, by molarity. 
To prepare fibronectin-coated beads (positive-control for glycan coated beads), fibronectin (FN, Cat \# 354008, Sigma-Aldrich, St. Louis, MO) was first biotinylated by incubating $45 \mu \mathrm{l}$ of $10 \mathrm{mM}$ biotin with $1 \mathrm{ml}$ of a $1 \mathrm{mg} / \mathrm{ml}$ solution of $\mathrm{FN}$, both in PBS. After incubation for $2 \mathrm{~h}$ at $5{ }^{\circ} \mathrm{C}$, free biotin was removed using a desalting spin column. The biotinylated FN $(60 \mu \mathrm{g})$ was incubated with $20 \mu \mathrm{L}$ of streptavidinSepharose beads for $90 \mathrm{~min}$ at room temperature as above for biotinylated glycans.

Beads incubated with biotinylated FN and glycans were washed twice in NC-TALP and re-suspended in $100 \mu \mathrm{l}$ of NC-TALP (non-capacitating mTALP; NC-TALP; 2.1 $\mathrm{mM} \mathrm{CaCl}_{2}, 3.1 \mathrm{mM} \mathrm{KCl}, 1.5 \mathrm{mM} \mathrm{MgCl}_{2}, 100 \mathrm{mM} \mathrm{NaCl}, 0.29 \mathrm{mM} \mathrm{KH}_{2} \mathrm{PO}_{4}, 0.36 \%$ lactic acid, $0.6 \%$ polyvinyl alcohol, $1 \mathrm{mM}$ pyruvic acid, $35 \mathrm{mM}$ HEPES, [pH 7.3], sterile filtered). Once the glycan-coupled beads were ready for use, a $50 \mu \mathrm{L}$ droplet containing 1.5 million spermatozoa/mL was prepared to receive $1 \mu \mathrm{L}$ of glycancoated beads. Non-capacitated spermatozoa and beads were co-incubated for 45 min at $39^{\circ} \mathrm{C}, 0 \% \mathrm{CO}_{2}$. Number of spermatozoa bound per bead was counted before addition of any treatments. After that, an appropriate amount of zinc was added $\left(\mathrm{ZnCl}_{2} ; 15 \mu \mathrm{L}, 0.5 \mathrm{mM}, 1.5 \mathrm{mM}, 2.5 \mathrm{mM}\right)$; as controls for zinc, calcium $\left(\mathrm{CaCl}_{2}\right.$; $2.5 \mathrm{mM})$ and magnesium $\left(\mathrm{MgCl}_{2} ; 2.5 \mathrm{mM}\right)$ were added. After incubation for $15 \mathrm{~min}$ at $39^{\circ} \mathrm{C}, 0 \% \mathrm{CO}_{2}$, progesterone $\left(\mathrm{P}_{4} ; 80 \mathrm{nM}\right)$ was added to the droplets and incubated for $30 \mathrm{~min}$ at $39^{\circ} \mathrm{C}, 0 \% \mathrm{CO}_{2}$. For each treatment, 25 beads were randomly selected and the total number of bound spermatozoa was enumerated. Spermatozoa that were self-agglutinated were not included in the counts. The 
experiment was documented using a Zeiss Axioskop and AxioCamHRc (Zeiss Microcopy, LLC,, Thornwood, NY, USA).

\section{Zymography Sample Preparation and Assay}

Boar and bull spermatozoa were prepared for zymography as previously described (Ferrer et al., 2012a), including use of the trophoblast cell line known to express MMP2 and MMP9. Briefly, detergents used to solubilize and extract inner acrosomal membrane (IAM) associated proteins were non-ionic detergent Nonidet P-40 (NP-40) with sonication followed by separate $1 \%$ SDS extraction. The supernatant was separated from the pellet by centrifugation at 7,000g for $10 \mathrm{~min}$ at $4{ }^{\circ} \mathrm{C}$ and the resultant fractions were mixed with non-reducing sample buffer (200 mM Tris pH 6.8, 4 \% SDS, $0.1 \%$ bromophenol blue, $40 \%$ glycerol) for analysis by zymography.

Samples were loaded onto $10 \%$ SDS-polyacrylamide gels containing gelatin as previously described (Ferrer et al., 2012a). After electrophoresis, enzymes were rinsed twice for 30 min and followed by an additional $1 \mathrm{~h}$ in $2.5 \%$ Triton X-100 ( $\mathrm{TrX}$ ), $5 \mathrm{mM} \mathrm{CaCl}_{2}, 50 \mathrm{mM}$ Tris $\mathrm{pH} 7.5$ in ddH2O at room temperature followed by overnight $37^{\circ} \mathrm{C}$ incubation in solution void of $\operatorname{TrX}$. MMP gelatinase activity was studied with and without inclusion of $1.5 \mathrm{mM} \mathrm{ZnCl}_{2}$ (with vehicle control already containing $5.0 \mathrm{mM} \mathrm{CaCl}_{2}$ ). The next day, gels were stained with Coomassie stain and destained in $30 \%$ methanol, $10 \%$ glacial acetic acid and $60 \%$ ddH $2 \mathrm{O}$ for 2 
hrs. Clear bands in the zymogram indicated enzymatic digestion of gelatin. Results shown are typical of two replicates.

\section{Statistics}

All results are presented as mean \pm standard error unless otherwise noted. Chapter 2 \& 4: Prism 8.0 one-way ANOVA was used to analyze the replicates. The means were considered to belong to distinct populations if $P<0.05$ using Tukey's test for multiple comparisons. Chapter 3: SAS 9.4 (SAS Institute, Inc, Cary, NC) GLM procedure and Duncan's Multiple Range test was used to analyze the replicates. Bartlett and Leven tests found the sample sets to be homogenous. 


\section{BIBLIOGRAPHY}

Acott, T. S., and D. W. Carr. 1984. Inhibition of bovine spermatozoa by caudal epididymal fluid: II. Interaction of $\mathrm{pH}$ and a quiescence factor. Biology of reproduction 30(4):926-935.

Aitken, R. J., M. A. Baker, and B. Nixon. 2015. Are sperm capacitation and apoptosis the opposite ends of a continuum driven by oxidative stress? Asian J Androl 17(4):633-639. doi: 10.4103/1008-682X.153850

Alvarez, J. G., J. C. Touchstone, L. Blasco, and B. T. Storey. 1987. Spontaneous lipid peroxidation and production of hydrogen peroxide and superoxide in human spermatozoa. Superoxide dismutase as major enzyme protectant against oxygen toxicity. Journal of andrology 8(5):338-348.

Alvau, A., M. A. Battistone, M. G. Gervasi, F. A. Navarrete, X. Xu, C. SánchezCárdenas, J. L. De la Vega-Beltran, V. G. Da Ros, P. A. Greer, A. Darszon, D. Krapf, A. M. Salicioni, P. S. Cuasnicu, and P. E. Visconti. 2016. The tyrosine kinase FER is responsible for the capacitationassociated increase in tyrosine phosphorylation in murine sperm. Development 143(13):2325-2333. doi: 10.1242/dev.136499

Amari, S., N. Yonezawa, S. Mitsui, T. Katsumata, S. Hamano, M. Kuwayama, Y. Hashimoto, A. Suzuki, Y. Takeda, and M. Nakano. 2001. Essential role of the nonreducing terminal alpha-mannosyl residues of the $\mathrm{N}$-linked carbohydrate chain of bovine zona pellucida glycoproteins in sperm-egg binding. Mol Reprod Dev 59(2):221-226. doi: 10.1002/mrd.1026 
Ambroggio, X. I., D. C. Rees, and R. J. Deshaies. 2003. JAMM: A Metalloprotease-Like Zinc Site in the Proteasome and Signalosome. PLOS Biology 2(1):e2. doi: 10.1371/journal.pbio.0020002

Amici, M., K. Forti, C. Nobili, G. Lupidi, M. Angeletti, E. Fioretti, and A. Eleuteri. 2002. Effect of neurotoxic metal ions on the proteolytic activities of the $20 \mathrm{~S}$ proteasome from bovine brain. JBIC Journal of Biological Inorganic Chemistry 7(7-8):750-756. doi: 10.1007/s00775-002-0352-4

Andreini, C., L. Banci, I. Bertini, and A. Rosato. 2006. Counting the zinc-proteins encoded in the human genome. Journal of proteome research 5(1):196201. doi: $10.1021 / p r 050361 \mathrm{j}$

Andrews, J. C., J. P. Nolan, R. H. Hammerstedt, and B. D. Bavister. 1994. Role of zinc during hamster sperm capacitation. Biology of reproduction 51(6):1238-1247.

Andreychenko, S. V., A. V. Klepko, L. V. Gorban, O. A. Motryna, L. V. Grubska, and O. V. Trofimenko. 2016. Post-Chornobyl remote radiation effects on human sperm and seminal plasma characteristics. Experimental oncology $38(4): 245-251$.

Araki, N., G. Trencsenyi, Z. T. Krasznai, E. Nizsaloczki, A. Sakamoto, N. Kawano, K. Miyado, K. Yoshida, and M. Yoshida. 2015. Seminal Vesicle Secretion 2 Acts as a Protectant of Sperm Sterols and Prevents Ectopic Sperm Capacitation in Mice. Biology of reproduction 92(1):8-8. doi: 10.1095/biolreprod.114.120642 
Arangasamy, A., M. Venkata Krishnaiah, N. Manohar, S. Selvaraju, P. R.

Guvvala, N. M. Soren, I. J. Reddy, K. S. Roy, and J. P. Ravindra. 2018. Advancement of puberty and enhancement of seminal characteristics by supplementation of trace minerals to bucks. Theriogenology 110:182-191. doi: 10.1016/j.theriogenology.2018.01.008

Arcelay, E., A. M. Salicioni, E. Wertheimer, and P. E. Visconti. 2008. Identification of proteins undergoing tyrosine phosphorylation during mouse sperm capacitation. International Journal of Developmental Biology 52(5-6):463-472. doi: 10.1387/ijdb.072555ea

Arver, S. 1980. Zinc and zinc ligands in human seminal plasma. I. Methodological aspects and normal findings. International journal of andrology 3(6):629-642.

Arver, S. 1982. Zinc and zinc ligands in human seminal plasma. III. The principal low molecular weight zinc ligand in prostatic secretion and seminal plasma. Acta physiologica Scandinavica 116(1):67-73. doi:

10.1111/j.1748-1716.1982.tb10600.x

Arver, S., and R. Eliasson. 1982. Zinc and zinc ligands in human seminal plasma. II. Contribution by ligands of different origin to the zinc binding properties of human seminal plasma. Acta physiologica Scandinavica 115(2):217-224. doi: 10.1111/j.1748-1716.1982.tb07068.x

Atabakhsh, M., I. Khodadadi, I. Amiri, H. Mahjub, and H. Tavilani. 2018. Activity of Matrix Metalloproteinase 2 and 9 in Follicular Fluid and Seminal Plasma 
and Its Relation to Embryo Quality and Fertilization Rate. Journal of reproduction \& infertility 19(3):140-145.

Austin, C. R. 1951. Observations on the penetration of the sperm in the mammalian egg. Australian journal of scientific research. Ser. B: Biological sciences 4(4):581-596.

Austin, C. R. 1952. The capacitation of the mammalian sperm. Nature 170(4321):326

Baba, T., S. Azuma, S. Kashiwabara, and Y. Toyoda. 1994. Sperm from mice carrying a targeted mutation of the acrosin gene can penetrate the oocyte zona pellucida and effect fertilization. The Journal of biological chemistry 269(50):31845-31849.

Babcock, D. F., G. A. Rufo, Jr., and H. A. Lardy. 1983. Potassium-dependent increases in cytosolic pH stimulate metabolism and motility of mammalian sperm. Proc Natl Acad Sci U S A 80(5):1327-1331.

Baccetti, B., V. Pallini, and A. G. Burrini. 1976. The accessory fibers of the sperm tail. II. Their role in binding zinc in mammals and cephalopods. Journal of ultrastructure research 54(2):261-275.

Backstrom, J. R., C. A. Miller, and Z. A. Tokes. 1992. Characterization of neutral proteinases from Alzheimer-affected and control brain specimens: identification of calcium-dependent metalloproteinases from the hippocampus. Journal of neurochemistry 58(3):983-992. doi: 10.1111/j.1471-4159.1992.tb09352.x 
Baker, M. A., L. Hetherington, and R. J. Aitken. 2006. Identification of SRC as a key PKA-stimulated tyrosine kinase involved in the capacitationassociated hyperactivation of murine spermatozoa. J Cell Sci 119(Pt 15):3182-3192. doi: 10.1242/jcs.03055

Baker, M. A., G. Reeves, L. Hetherington, and R. J. Aitken. 2010a. Analysis of proteomic changes associated with sperm capacitation through the combined use of IPG-strip pre-fractionation followed by RP chromatography LC-MS/MS analysis. Proteomics 10(3):482-495. doi: 10.1002/pmic. 200900574

Baker, M. A., N. D. Smith, L. Hetherington, K. Taubman, M. E. Graham, P. J. Robinson, and R. J. Aitken. 2010b. Label-free quantitation of phosphopeptide changes during rat sperm capacitation. Journal of proteome research 9(2):718-729. doi: 10.1021/pr900513d

Barceloux, D. G., and D. Barceloux. 1999. Zinc. Journal of Toxicology: Clinical Toxicology 37(2):279-292. doi: 10.1081/CLT-100102426

Barney, G. H., M. C. Orgebin-Crist, and M. P. Macapinalac. 1968. Genesis of esophageal parakeratosis and histologic changes in the testes of the zincdeficient rat and their reversal by zinc repletion. The Journal of nutrition 95(4):526-534. doi: 10.1093/jn/95.4.526

Beconi, M. T., C. R. Francia, N. G. Mora, and M. A. Affranchino. 1993. Effect of natural antioxidants on frozen bovine semen preservation. Theriogenology 40(4):841-851. 
Bedford, J. M., and H. I. Calvin. 1974. Changes in -S-S- linked structures of the sperm tail during epididymal maturation, with comparative observations in sub-mammalian species. The Journal of experimental zoology 187(2):181204. doi: $10.1002 /$ jez. 1401870202

Beek, J., H. Nauwynck, D. Maes, and A. Van Soom. 2012. Inhibitors of zincdependent metalloproteases hinder sperm passage through the cumulus oophorus during porcine fertilization in vitro. Reproduction 144(6):687-697. doi: 10.1530/REP-12-0311

Bell, D. J., and P. E. Lake. 1962. A comparison of phosphomonesterase activities in the seminal plasmas of the domestic cock, turkey tom, boar, bull, buck rabbit and of man. J Reprod Fertil 3:363-368.

Bentley, P. J., and B. R. Grubb. 1991. Effects of a zinc-deficient diet on tissue zinc concentrations in rabbits. Journal of animal science 69(12):48764882.

Berruti, G. 2016. Towards defining an 'origin' - The case for the mammalian acrosome. Semin Cell Dev Biol doi: S1084-9521(16)30013-1 [pii]

10.1016/j.semcdb.2016.01.013

Bettger, W. J., and B. L. O'Dell. 1981. A critical physiological role of zinc in the structure and function of biomembranes. Life sciences 28(13):1425-1438.

Beyersmann, D., and H. Haase. 2001. Functions of zinc in signaling, proliferation and differentiation of mammalian cells. Biometals : an international journal on the role of metal ions in biology, biochemistry, and medicine 14(34):331-341. 
Bianchi, F., R. Rousseaux-Prevost, P. Sautiere, and J. Rousseaux. 1992. P2 protamines from human sperm are zinc -finger proteins with one CYS2/HIS2 motif. Biochemical and biophysical research communications 182(2):540-547. doi: 10.1016/0006-291X(92)91766-J

Bjorndahl, L., S. Kjellberg, G. M. Roomans, and U. Kvist. 1986. The human sperm nucleus takes up zinc at ejaculation. International journal of andrology 9(1):77-80.

Bjorndahl, L., and U. Kvist. 2003. Sequence of ejaculation affects the spermatozoon as a carrier and its message. Reproductive biomedicine online 7(4):440-448.

Bjorndahl, L., and U. Kvist. 2010. Human sperm chromatin stabilization: a proposed model including zinc bridges. Molecular human reproduction 16(1):23-29. doi: 10.1093/molehr/gap099

Bjorndahl, L., and U. Kvist. 2011. A model for the importance of zinc in the dynamics of human sperm chromatin stabilization after ejaculation in relation to sperm DNA vulnerability. Syst Biol Reprod Med 57(1-2):86-92. doi: 10.3109/19396368.2010.516306

Bjorndahl, L., and U. Kvist. 2014. Structure of chromatin in spermatozoa. Adv Exp Med Biol 791:1-11. doi: 10.1007/978-1-4614-7783-9_1

Blindauer, C. A., and O. I. Leszczyszyn. 2010. Metallothioneins: unparalleled diversity in structures and functions for metal ion homeostasis and more. Natural product reports 27(5):720-741. doi: 10.1039/b906685n 
Boatman, D. E., and R. S. Robbins. 1991. Bicarbonate: carbon-dioxide regulation of sperm capacitation, hyperactivated motility, and acrosome reactions. Biology of reproduction 44(5):806-813.

Boerke, A., P. S. Tsai, N. Garcia-Gil, I. A. Brewis, and B. M. Gadella. 2008. Capacitation-dependent reorganization of microdomains in the apical sperm head plasma membrane: functional relationship with zona binding and the zona-induced acrosome reaction. Theriogenology 70(8):11881196. doi: 10.1016/j.theriogenology.2008.06.021

Bohring, C., E. Krause, B. Habermann, and W. Krause. 2001. Isolation and identification of sperm membrane antigens recognized by antisperm antibodies, and their possible role in immunological infertility disease. Mol Hum Reprod 7(2):113-118.

Bose, S., G. G. Mason, and A. J. Rivett. 1999. Phosphorylation of proteasomes in mammalian cells. Molecular biology reports 26(1-2):11-14.

Boursnell, J. C., S. Baronos, P. A. Briggs, and E. J. Butler. 1972. The concentrations of zinc in boar seminal plasma and vesicular secretion in relation to those of nitrogenous substances, citrate, galactose and fructose. J Reprod Fertil 29(2):215-227.

Bray, T. M., and W. J. Bettger. 1990. The physiological role of zinc as an antioxidant. Free radical biology \& medicine 8(3):281-291.

Brentjens, J. R., S. Matsuo, G. A. Andres, P. R. Caldwell, and L. Zamboni. 1986. Gametes contain angiotensin converting enzyme (kininase II). Experientia 42(4):399-402. 
Brssow, K. P., J. Rtky, and H. Rodriguez-Martinez. 2008. Fertilization and Early Embryonic Development in the Porcine Fallopian Tube. 43:245-251. doi: 10.1111/j.1439-0531.2008.01169.x

Bucci, D., G. Isani, E. Giaretta, M. Spinaci, C. Tamanini, E. Ferlizza, and G. Galeati. 2014. Alkaline phosphatase in boar sperm function. Andrology 2(1):100-106. doi: 10.1111/j.2047-2927.2013.00159.x

Buchman-Shaked, O., Z. Kraiem, Y. Gonen, and S. Goldman. 2002. Presence of matrix metalloproteinases and tissue inhibitor of matrix metalloproteinase in human sperm. Journal of andrology 23(5):702-708.

Buffone, M. G., J. C. Calamera, S. V. Verstraeten, and G. F. Doncel. 2005. Capacitation-associated protein tyrosine phosphorylation and membrane fluidity changes are impaired in the spermatozoa of asthenozoospermic patients. Reproduction 129(6):697-705. doi: 10.1530/rep.1.00584

Buffone, M. G., T. W. Ijiri, W. Cao, T. Merdiushev, H. K. Aghajanian, and G. L. Gerton. 2012. Heads or tails? Structural events and molecular mechanisms that promote mammalian sperm acrosomal exocytosis and motility. Mol Reprod Dev 79(1):4-18. doi: 10.1002/mrd.21393

Campanero-Rhodes, M. A., M. Menéndez, J. L. Sáiz, L. Sanz, J. J. Calvete, and D. Solís. 2006. Zinc lons Induce the Unfolding and Self-Association of Boar Spermadhesin PSP-I, a Protein with a Single CUB Domain Architecture, and Promote Its Binding to Heparin†. Biochemistry 45(27):8227-8235. doi: 10.1021/bi052621g 
Carrasco, L. C., P. Coy, M. Aviles, J. Gadea, and R. Romar. 2008a. Glycosidase determination in bovine oviducal fluid at the follicular and luteal phases of the oestrous cycle. Reprod Fertil Dev 20(7):808-817.

Carrasco, L. C., R. Romar, M. Aviles, J. Gadea, and P. Coy. 2008b.

Determination of glycosidase activity in porcine oviductal fluid at the different phases of the estrous cycle. Reproduction 136(6):833-842. doi: 10.1530/rep-08-0221

Chakravarty, S., P. Bansal, P. Sutovsky, and S. K. Gupta. 2008. Role of proteasomal activity in the induction of acrosomal exocytosis in human spermatozoa. Reproductive biomedicine online 16(3):391-400.

Chang, M. C. 1951. Fertilizing capacity of spermatozoa deposited into the fallopian tubes. Nature 168(4277):697-698.

Chi, A., and R. G. Kemp. 2000. The primordial high energy compound: ATP or inorganic pyrophosphate? The Journal of biological chemistry 275(46):35677-35679. doi: 10.1074/jbc.C000581200

Chi, Z. H., W. Y. Feng, H. L. Gao, W. Zheng, L. Huang, and Z. Y. Wang. 2009. ZNT7 and Zn2+ are present in different cell populations in the mouse testis. Histology and histopathology 24(1):25-30. doi: 10.14670/hh-24.25

Cho, C. 2012. Testicular and epididymal ADAMs: expression and function during fertilization. Nature reviews. Urology 9(10):550-560. doi: 10.1038/nrurol.2012.167

Choi, Y.-J., S.-J. Uhm, S.-J. Song, H. Song, J.-K. Park, T. Kim, C. Park, and J.-H. Kim. 2008. Cytochrome c upregulation during capacitation and 
spontaneous acrosome reaction determines the fate of pig sperm cells: Linking proteome analysis. Journal of Reproduction and Development 54(1):68-83. doi: 10.1262/jrd.19116

Chu, D. S. 2018. Zinc: A small molecule with a big impact on sperm function. PLoS Biol 16(6):e2006204. doi: 10.1371/journal.pbio.2006204 Chunghee, C. 2005. Mammalian ADAMs with Testis-Specific or -Predominant Expression. In: N. Hooper and U. Lendeckel, editors, The ADAM Family of Proteases. Springer, Dordrecht. p. 239-259.

Ciereszko, A., K. Dabrowski, S. D. Mims, and J. Glogowski. 2000. Characteristics of sperm acrosin-like activity of paddlefish (Polyodon spathula Walbaum). Comparative biochemistry and physiology. Part B, Biochemistry \& molecular biology 125(2):197-203. doi: 10.1016/S0305-0491(99)00167-4

Clapper, D. L., J. A. Davis, P. J. Lamothe, C. Patton, and D. Epel. 1985. Involvement of zinc in the regulation of $\mathrm{pHi}$, motility, and acrosome reactions in sea urchin sperm. The Journal of cell biology 100(6):18171824. doi: $10.1083 /$ jcb.100.6.1817

Colagar, A. H., E. T. Marzony, and M. J. Chaichi. 2009. Zinc levels in seminal plasma are associated with sperm quality in fertile and infertile men. Nutrition research (New York, N.Y.) 29(2):82-88. doi: 10.1016/j.nutres.2008.11.007

Coleman, J. E. 1992. Zinc proteins: enzymes, storage proteins, transcription factors, and replication proteins. Annual review of biochemistry 61:897946. doi: 10.1146/annurev.bi.61.070192.004341 
Coyle, P., J. C. Philcox, L. C. Carey, and A. M. Rofe. 2002. Metallothionein: the multipurpose protein. Cellular and molecular life sciences : CMLS 59(4):627-647.

Crawford, G., A. Ray, A. Gudi, A. Shah, and R. Homburg. 2015. The role of seminal plasma for improved outcomes during in vitro fertilization treatment: review of the literature and meta-analysis. 21(2):275-284. doi: 10.1093/humupd/dmu052

Croxford, T. P., N. H. McCormick, and S. L. Kelleher. 2011. Moderate zinc deficiency reduces testicular Zip6 and Zip10 abundance and impairs spermatogenesis in mice. The Journal of nutrition 141(3):359-365. doi: 10.3945/jn.110.131318

Cui, N., M. Hu, and R. A. Khalil. 2017. Biochemical and Biological Attributes of Matrix Metalloproteinases. Progress in molecular biology and translational science 147:1-73. doi: 10.1016/bs.pmbts.2017.02.005

Dafeldecker, W. P., and B. L. Vallee. 1986. Organ-specific human alcohol dehydrogenase: isolation and characterization of isozymes from testis. Biochemical and biophysical research communications 134(3):1056-1063.

Dai, R. M., and C. C. Li. 2001. Valosin-containing protein is a multi-ubiquitin chain-targeting factor required in ubiquitin-proteasome degradation. Nature cell biology 3(8):740-744. doi: 10.1038/35087056

Davis, B. K. 1981. Timing of fertilization in mammals: sperm cholesterol/phospholipid ratio as a determinant of the capacitation interval. Proc Natl Acad Sci U S A 78(12):7560-7564. 
De Jonge, C. 2017. Biological basis for human capacitation-revisited. Hum Reprod Update 23(3):289-299. doi: 10.1093/humupd/dmw048

Demott, R. P., and S. S. Suarez. 1992. Hyperactivated sperm progress in the mouse oviduct. Biology of reproduction 46(5):779-785.

Dhanda, O. P., B. R. Rao, and M. N. Razdan. 1981. Sorbitol dehydrogenase \& hyaluronidase activity in buffalo semen. Indian journal of experimental biology 19(3):286.

Diaz, E. S., M. Kong, and P. Morales. 2007. Effect of fibronectin on proteasome activity, acrosome reaction, tyrosine phosphorylation and intracellular calcium concentrations of human sperm. Human reproduction 22(5):14201430. doi: $10.1093 /$ humrep/dem023

Ding, B., and Q. Zhong. 2017. Zinc deficiency: An unexpected trigger for autophagy. The Journal of biological chemistry 292(20):8531-8532. doi: 10.1074/jbc.H116.762948

Dissanayake, D., P. S. Wijesinghe, W. D. Ratnasooriya, and S. Wimalasena. 2009. Effects of zinc supplementation on sexual behavior of male rats. Journal of Human Reproductive Sciences 2(2):57-61. doi: 10.4103/09741208.57223

Dobrinski, I., G. G. Ignotz, M. S. Fagnan, S. I. Yudin, and B. A. Ball. 1997. Isolation and characterization of a protein with homology to angiotensin converting enzyme from the periacrosomal plasma membrane of equine spermatozoa. Molecular reproduction and development 48(2):251-260. doi: 10.1002/(sici)1098-2795(199710)48:2<251::Aid-mrd13>3.0.Co;2-0 
Duan, C., and E. Goldberg. 2003. Inhibition of lactate dehydrogenase C4 (LDHC4) blocks capacitation of mouse sperm in vitro. Cytogenetic and genome research 103(3-4):352-359. doi: 10.1159/000076824

Dube, C., S. Tardif, P. LeClerc, and J. L. Bailey. 2003. The importance of calcium in the appearance of p32, a boar sperm tyrosine phosphoprotein, during in vitro capacitation. J Androl 24(5):727-733.

Einarsson, S., B. Gustafsson, and I. Settergren. 1976. Alkaline phosphatase activity of epididymal contents in boars with normal or reduced spermatogenesis. Andrologia 8(1):25-28.

Ekhlasi-Hundrieser, M., K. Gohr, A. Wagner, M. Tsolova, A. Petrunkina, and E. Topfer-Petersen. 2005. Spermadhesin AQN1 is a candidate receptor molecule involved in the formation of the oviductal sperm reservoir in the pig. Biology of reproduction 73(3):536-545. doi:

10.1095/biolreprod.105.040824

Elgazar, V., V. Razanov, M. Stoltenberg, M. Hershfinkel, M. Huleihel, Y. B. Nitzan, E. Lunenfeld, I. Sekler, and W. F. Silverman. 2005. Zinc-regulating proteins, $\mathrm{ZnT}-1$, and metallothionein I/II are present in different cell populations in the mouse testis. The journal of histochemistry and cytochemistry : official journal of the Histochemistry Society 53(7):905912. doi: 10.1369/jhc.4A6482.2005

Ensslin, M., T. Vogel, J. J. Calvete, H. H. Thole, J. Schmidtke, T. Matsuda, and E. Töpfer-Petersen. 1998. Molecular cloning and characterization of P47, a novel boar sperm-associated zona pellucida-binding protein homologous 
to a family of mammalian secretory proteins. Biol Reprod 58(4):10571064. doi: 10.1095/biolreprod58.4.1057

Flesch, J. F. H. M. B. F.M., P.F.E.M. Nievelstein, A.J. Verkleij, L.M.G. van Golde, B. Colenbrander, and B.M. Gadella. 2001. Bicarbonate stimulated phospholipid scrambling induces cholesterol redistribution and enables cholesterol depletion in the sperm plasma membrane. Journal of Cell Science 114:3543-3555.

Fallah, A., A. Mohammad-Hasani, and A. H. Colagar. 2018. Zinc is an Essential Element for Male Fertility: A Review of Zn Roles in Men's Health, Germination, Sperm Quality, and Fertilization. Journal of Reproduction \& Infertility 19(2):069-080.

Ferrer, M., H. Rodriguez, L. Zara, Y. Yu, W. Xu, and R. Oko. 2012a. MMP2 and acrosin are major proteinases associated with the inner acrosomal membrane and may cooperate in sperm penetration of the zona pellucida during fertilization. Cell and Tissue Research 349(3):881-895. doi: $10.1007 / \mathrm{s} 00441-012-1429-1$

Ferrer, M., H. Rodriguez, L. Zara, Y. Yu, W. Xu, and R. Oko. 2012b. MMP2 and acrosin are major proteinases associated with the inner acrosomal membrane and may cooperate in sperm penetration of the zona pellucida during fertilization. Cell and tissue research 349(3):881-895. doi: $10.1007 / \mathrm{s} 00441-012-1429-1$

Ficarro, S., O. Chertihin, V. A. Westbrook, F. White, F. Jayes, P. Kalab, J. A. Marto, J. Shabanowitz, J. C. Herr, D. F. Hunt, and P. E. Visconti. 2003. 
Phosphoproteome analysis of capacitated human sperm. Evidence of tyrosine phosphorylation of a kinase-anchoring protein 3 and valosincontaining protein/p97 during capacitation. The Journal of biological chemistry 278(13):11579-11589. doi: 10.1074/jbc.M202325200

Flesch, F. M., B. Colenbrander, L. M. G. van Golde, and B. M. Gadella. 1999. Capacitation Induces Tyrosine Phosphorylation of Proteins in the Boar Sperm Plasma Membrane. Biochemical and biophysical research communications 262(3):787-792. doi: 10.1006/bbrc.1999.1300

Florman, H. M., M. K. Jungnickel, and K. A. Sutton. 2010. Shedding light on sperm pHertility. Cell 140(3):310-312. doi: 10.1016/j.cell.2010.01.035

Foresta, C., A. Garolla, I. Cosci, M. Menegazzo, M. Ferigo, V. Gandin, and L. De Toni. 2014. Role of zinc trafficking in male fertility: from germ to sperm. Human reproduction 29(6):1134-1145. doi: 10.1093/humrep/deu075

Fraser, L. R. 1998. Interactions between a decapacitation factor and mouse spermatozoa appear to involve fucose residues and a GPI-anchored receptor. Mol Reprod Dev 51(2):193-202. doi: 10.1002/(sici)10982795(199810)51:2<193::aid-mrd9>3.0.co;2-L

Fu, G., Y. Wei, X. Wang, and L. Yu. 2015. Identification of candidate causal genes and their associated pathogenic mechanisms underlying teratozoospermia based on the spermatozoa transcript profiles. Andrologia:n/a-n/a. doi: 10.1111/and.12484 
Gadella, B. M., P. S. Tsai, A. Boerke, and I. A. Brewis. 2008. Sperm head membrane reorganisation during capacitation. Int J Dev Biol 52(5-6):473480. doi: $082583 \mathrm{bg}$

Gatti, J. L., X. Druart, Y. Guerin, F. Dacheux, and J. L. Dacheux. 1999. A 105- to 94-kilodalton protein in the epididymal fluids of domestic mammals is angiotensin I-converting enzyme (ACE); evidence that sperm are the source of this ACE. Biology of reproduction 60(4):937-945.

Gavella, M., and P. Cvitkovic. 1985. Semen LDH-X deficiency and male infertility. Archives of andrology 15(2-3):173-176.

Gervasi, M. G., and P. E. Visconti. 2016. Chang's meaning of capacitation: A molecular perspective. Mol Reprod Dev 83(10):860-874. doi: $10.1002 / \mathrm{mrd} .22663$

Geussova, G., P. Kalab, and J. Peknicova. 2002. Valosine containing protein is a substrate of cAMP-activated boar sperm tyrosine kinase. Mol Reprod Dev 63(3):366-375. doi: 10.1002/mrd.10156

Giachi, G., P. Pallecchi, A. Romualdi, E. Ribechini, J. J. Lucejko, M. P. Colombini, and M. Mariotti Lippi. 2013. Ingredients of a 2,000-y-old medicine revealed by chemical, mineralogical, and botanical investigations. Proc Natl Acad Sci U S A 110(4):1193-1196. doi: 10.1073/pnas. 1216776110

Gibbons, R., S. A. Adeoya-Osiguwa, and L. R. Fraser. 2005. A mouse sperm decapacitation factor receptor is phosphatidylethanolamine-binding protein 1. Reproduction 130(4):497-508. doi: 10.1530/rep.1.00792 
Gillis, B. A., and T. M. Tamblyn. 1984. Association of bovine sperm aldolase with sperm subcellular components. Biology of reproduction 31(1):25-35.

Giojalas, L. C., R. A. Rovasio, G. Fabro, A. Gakamsky, and M. Eisenbach. 2004. Timing of sperm capacitation appears to be programmed according to egg availability in the female genital tract. Fertility and sterility 82(1):247-249. doi: 10.1016/j.fertnstert.2003.11.046

Goto, N., and H. Harayama. 2009. Calyculin A-sensitive protein phosphatases are involved in maintenance of progressive movement in mouse spermatozoa in vitro by suppression of autophosphorylation of protein kinase A. J Reprod Dev 55(3):327-334.

Gualtieri, R., V. Barbato, I. Fiorentino, S. Braun, D. Rizos, S. Longobardi, and R. Talevi. 2014. Treatment with zinc, d-aspartate, and coenzyme Q10 protects bull sperm against damage and improves their ability to support embryo development. Theriogenology 82(4):592-598. doi: https://doi.org/10.1016/j.theriogenology.2014.05.028

Guidobaldi, H. A., M. Cubilla, A. Moreno, M. V. Molino, L. Bahamondes, and L. C. Giojalas. 2017. Sperm chemorepulsion, a supplementary mechanism to regulate fertilization. Human reproduction 32(8):1560-1573. doi: 10.1093/humrep/dex232

Hadwan, M. H., L. A. Almashhedy, and A. R. Alsalman. 2015. Oral Zinc Supplementation Restores Superoxide Radical Scavengers to Normal Levels in Spermatozoa of Iraqi Asthenospermic Patients. International journal for vitamin and nutrition research. Internationale Zeitschrift fur 
Vitamin- und Ernahrungsforschung. Journal international de vitaminologie et de nutrition 85(3-4):165-173. doi: 10.1024/0300-9831/a000235

Harrison, R. A., H. M. Dott, and G. C. Foster. 1978. Effect of ionic strength, serum albumin and other macromolecules on the maintenance of motility and the surface of mammalian spermatozoa in a simple medium. $\mathrm{J}$ Reprod Fertil 52(1):65-73. doi: 10.1530/jrf.0.0520065

He, L. S., X. S. Yan, and D. C. Wu. 1991. Age-dependent variation of zinc-65 metabolism in LACA mice. International journal of radiation biology 60(6):907-916.

Hebles, M., M. Dorado, M. Gallardo, M. González-Martínez, and P. SánchezMartín. 2015. Seminal quality in the first fraction of ejaculate. Systems Biology in Reproductive Medicine 61(2):113-116. doi: $10.3109 / 19396368.2014 .999390$

Henkel, R., C. Baldauf, J. Bittner, W. Weidner, and W. Miska. 2001. Elimination of zinc from the flagella of spermatozoa during epididymal transit is important for motility. Reprod Technol 10(5):6.

Henkel, R., J. Bittner, R. Weber, F. Hüther, and W. Miska. 1999. Relevance of zinc in human sperm flagella and its relation to motility. Fertility and sterility 71(6):1138-1143. doi: 10.1016/S0015-0282(99)00141-7

Hershko, A., and A. Ciechanover. 1992. The ubiquitin system for protein degradation. Annual review of biochemistry 61:761-807. doi: 10.1146/annurev.bi.61.070192.003553 
Hillman, P., D. Ickowicz, R. Vizel, and H. Breitbart. 2013. Dissociation between AKAP3 and PKARII promotes AKAP3 degradation in sperm capacitation. PloS one 8(7):e68873. doi: 10.1371/journal.pone.0068873

Ho, H. C., K. A. Granish, and S. S. Suarez. 2002. Hyperactivated motility of bull sperm is triggered at the axoneme by $\mathrm{Ca} 2+$ and not cAMP. Dev Biol 250(1):208-217.

Holt, W. V., and R. D. North. 1986. Thermotropic phase transitions in the plasma membrane of ram spermatozoa. J Reprod Fertil 78(2):447-457. doi: $10.1530 /$ jrf.0.0780447

Hoshi, M., T. Numakunai, and H. Sawada. 1981. Evidence for participation of sperm proteinases in fertilization of the solitary ascidian, Halocynthia roretzi: effects of protease inhibitors. Dev Biol 86(1):117-121.

Huang, S. T., R. C. Yang, H. T. Wu, C. N. Wang, and J. H. Pang. 2011. Zincchelation contributes to the anti-angiogenic effect of ellagic acid on inhibiting MMP-2 activity, cell migration and tube formation. PloS one 6(5):e18986. doi: 10.1371/journal.pone.0018986

Huggins, C., and A. Johnson. 1933. Chemical observation on fluids of the seminal tract. American Journal of Physiology 1933(103):574-581.

Hung, P. H., and S. S. Suarez. 2010. Regulation of sperm storage and movement in the ruminant oviduct. Soc Reprod Fertil Suppl 67:257-266.

Hung, P. H., and S. S. Suarez. 2012. Alterations to the bull sperm surface proteins that bind sperm to oviductal epithelium. Biology of reproduction 87(4):88. doi: biolreprod.112.099721 [pii] 
10.1095/biolreprod.112.099721

Husnjak, K., S. Elsasser, N. Zhang, X. Chen, L. Randles, Y. Shi, K. Hofmann, K. J. Walters, D. Finley, and I. Dikic. 2008. Proteasome subunit Rpn13 is a novel ubiquitin receptor. Nature 453(7194):481-488. doi:

$10.1038 /$ nature06926

Ignotz, G. G., M. Y. Cho, and S. S. Suarez. 2007. Annexins are candidate oviductal receptors for bovine sperm surface proteins and thus may serve to hold bovine sperm in the oviductal reservoir. Biology of reproduction 77(6):906-913. doi: 10.1095/biolreprod.107.062505

Irani, M., M. Amirian, R. Sadeghi, J. L. Lez, and R. Latifnejad Roudsari. 2017. The Effect of Folate and Folate Plus Zinc Supplementation on Endocrine Parameters and Sperm Characteristics in Sub-Fertile Men: A Systematic Review and Meta-Analysis. Urology journal 14(5):4069-4078.

Jaiswal, A., P. Joshi, M. V. Kumar, J. N. Panda, and L. N. Singh. 1984. Angiotensin converting enzyme in the testis and epididymis of mammals. Andrologia 16(5):410-416.

Jaiswal, B. S., and M. Eisenbach. 2002. Capacitation. In: D. M. Hardy, editor, Fertilization. Academic Press. p. 57-117.

Johnsen, O., R. Eliasson, and C. O. Lofman. 1982. Inhibition of the gelatinolytic and esterolytic activity of human sperm acrosin by zinc. Acta physiologica Scandinavica 114(3):475-476. doi: 10.1111/j.1748-1716.1982.tb07013.x Johnson, F. O., E. T. Gilbreath, L. Ogden, T. C. Graham, and S. Gorham. 2011. Reproductive and developmental toxicities of zinc supplemented rats. 
Reproductive toxicology (Elmsford, N.Y.) 31(2):134-143. doi:

10.1016/j.reprotox.2010.10.009

Johnson, L., S. Wikström, and G. Nylander. 1969. The Vehicle for Zinc in the

Prostatic Secretion of Dogs. Scandinavian Journal of Urology and

Nephrology 3(1):9-11. doi: 10.3109/00365596909135373

Johnson, L. A., J. G. Aalbers, and H. G. Grooten. 1988. Artificial Insemination of Swine: Fecundity of Boar Semen Stored in Beltsville TS (BTS), Modified Modena (MM), or MR-A and Inseminated on One, Three and Four Days After Collection. Reproduction in Domestic Animals 23(2):49-55. (Article) doi: 10.1111/j.1439-0531.1988.tb01080.x

Johnson, L. A., K. F. Weitze, P. Fiser, and W. M. Maxwell. 2000. Storage of boar semen. Anim Reprod Sci 62(1-3):143-172.

Jonakova, V., M. Kraus, L. Veselsky, D. Cechova, K. Bezouska, and M. Ticha. 1998. Spermadhesins of the AQN and AWN families, DQH sperm surface protein and HNK protein in the heparin-binding fraction of boar seminal plasma. J Reprod Fertil 114(1):25-34.

Jones, M. M., J. E. Schoenheit, and A. D. Weaver. 1979. Pretreatment and heavy metal LD50 values. Toxicology and applied pharmacology 49(1):4144.

Kaelberer, M. M., K. L. Buchanan, M. E. Klein, B. B. Barth, M. M. Montoya, X. Shen, and D. V. Bohórquez. 2018. A gut-brain neural circuit for nutrient sensory transduction. Science (New York, N.Y.) 361(6408):eaat5236. doi: 10.1126/science.aat5236 
Kawano, N., N. Araki, K. Yoshida, T. Hibino, N. Ohnami, M. Makino, S. Kanai, H. Hasuwa, M. Yoshida, K. Miyado, and A. Umezawa. 2014. Seminal vesicle protein SVS2 is required for sperm survival in the uterus. P Natl Acad Sci USA 111(11):4145-4150. doi: 10.1073/pnas.1320715111

Kennedy, C. E., K. B. Krieger, M. Sutovsky, W. Xu, P. Vargovic, B. A. Didion, M. R. Ellersieck, M. E. Hennessy, J. Verstegen, R. Oko, and P. Sutovsky. 2014. Protein expression pattern of PAWP in bull spermatozoa is associated with sperm quality and fertility following artificial insemination. Mol Reprod Dev 81(5):436-449. doi: 10.1002/mrd.22309

Kerns, K., P. Morales, and P. Sutovsky. 2016. Regulation of Sperm Capacitation by the 26S Proteasome: An Emerging New Paradigm in Spermatology. Biology of reproduction 94(5):117. doi: 10.1095/biolreprod.115.136622

Kerns, K., M. Zigo, E. Z. Drobnis, M. Sutovsky, and P. Sutovsky. 2018a. Zinc ion flux during mammalian sperm capacitation. Nature Communications 9(1):2061. doi: 10.1038/s41467-018-04523-y

Kerns, K., M. Zigo, and P. Sutovsky. 2018b. Zinc: A Necessary lon for Mammalian Sperm Fertilization Competency. International Journal of Molecular Sciences 19(12):4097. doi: 10.3390/ijms19124097

Khokha, A. M., P. P. Voronov, and S. M. Zimatkin. 1994. [Immunoenzyme and immunohistochemical analysis of class III alcohol dehydrogenase from human testis]. Biokhimiia (Moscow, Russia) 59(7):997-1002. 
Kilic, M. 2007. Effect of fatiguing bicycle exercise on thyroid hormone and testosterone levels in sedentary males supplemented with oral zinc. Neuro endocrinology letters 28(5):681-685.

Kim, A. M., M. L. Bernhardt, B. Y. Kong, R. W. Ahn, S. Vogt, T. K. Woodruff, and T. V. O'Halloran. 2011. Zinc sparks are triggered by fertilization and facilitate cell cycle resumption in mammalian eggs. ACS chemical biology 6(7):716-723. doi: 10.1021/cb200084y

Kim, A. M., S. Vogt, T. V. O'Halloran, and T. K. Woodruff. 2010. Zinc availability regulates exit from meiosis in maturing mammalian oocytes. Nature chemical biology 6(9):674-681. doi: 10.1038/nchembio.419

Kim, I., C. H. Kim, J. H. Kim, J. Lee, J. J. Choi, Z. A. Chen, M. G. Lee, K. C. Chung, C. Y. Hsu, and Y. S. Ahn. 2004. Pyrrolidine dithiocarbamate and zinc inhibit proteasome-dependent proteolysis. Experimental cell research 298(1):229-238. doi: 10.1016/j.yexcr.2004.04.017

Knox, R. V. 2007. Semen Processing, Extending \& Storage for Artificial Insemination in Swine. University of Illinois, Extension Publication

Koch, S., S. P. Acebron, J. Herbst, G. Hatiboglu, and C. Niehrs. 2015. Posttranscriptional Wnt Signaling Governs Epididymal Sperm Maturation. Cell 163(5):1225-1236. doi: S0092-8674(15)01343-4 [pii]

10.1016/j.cell.2015.10.029

Kondoh, G., H. Tojo, Y. Nakatani, N. Komazawa, C. Murata, K. Yamagata, Y. Maeda, T. Kinoshita, M. Okabe, R. Taguchi, and J. Takeda. 2005. Angiotensin-converting enzyme is a GPI-anchored protein releasing factor 
crucial for fertilization. Nature medicine 11(2):160-166. doi:

$10.1038 / \mathrm{nm} 1179$

Kong, M., E. S. Diaz, and P. Morales. 2009. Participation of the human sperm proteasome in the capacitation process and its regulation by protein kinase A and tyrosine kinase. Biology of reproduction 80(5):1026-1035. doi: 10.1095/biolreprod.108.073924

Kongmanas, K., H. Kruevaisayawan, A. Saewu, C. Sugeng, J. Fernandes, P. Souda, J. B. Angel, K. F. Faull, R. J. Aitken, J. Whitelegge, D. Hardy, T. Berger, M. A. Baker, and N. Tanphaichitr. 2014. Proteomic Characterization of Pig Sperm Anterior Head Plasma Membrane Reveals Roles of Acrosomal Proteins in ZP3 Binding. Journal of cellular physiology doi: $10.1002 / j c p .24728$

Kongmanas, K., H. Kruevaisayawan, A. Saewu, C. Sugeng, J. Fernandes, P. Souda, J. B. Angel, K. F. Faull, R. J. Aitken, J. Whitelegge, D. Hardy, T. Berger, M. A. Baker, and N. Tanphaichitr. 2015. Proteomic characterization of pig sperm anterior head plasma membrane reveals roles of acrosomal proteins in ZP3 binding. Journal of cellular physiology 230(2):449-463. doi: 10.1002/jcp.24728

Kowalowka, M., P. Wysocki, L. Fraser, and J. Strzezek. 2008. Extracellular superoxide dismutase of boar seminal plasma. Reproduction in domestic animals $=$ Zuchthygiene 43(4):490-496. doi: 10.1111/j.14390531.2007.00943.x 
Krassnigg, F., H. Niederhauser, R. Placzek, J. Frick, and W. B. Schill. 1986. Investigations on the functional role of angiotensin converting enzyme (ACE) in human seminal plasma. Advances in experimental medicine and biology 198 Pt A:477-485.

Kratz, E. M., A. Kaluza, M. Ferens-Sieczkowska, B. Olejnik, R. Fiutek, M. Zimmer, and A. Piwowar. 2016. Gelatinases and their tissue inhibitors are associated with oxidative stress: a potential set of markers connected with male infertility. Reproduction, fertility, and development 28(7):1029-1037. doi: $10.1071 / \mathrm{rd} 14268$

Kvist, U. 1980a. Importance of spermatozoal zinc as temporary inhibitor of sperm nuclear chromatin decondensation ability in man. Acta physiologica Scandinavica 109(1):79-84. doi: 10.1111/j.1748-1716.1980.tb06567.x

Kvist, U. 1980b. Sperm nuclear chromatin decondensation ability. An in vitro study on ejaculated human spermatozoa. Acta physiologica Scandinavica. Supplementum 486:1-24.

Kvist, U., S. Kjellberg, L. Björndahl, J. C. Soufir, and S. Arver. 1990. Seminal fluid from men with agenesis of the Wolffian ducts: zinc-binding properties and effects on sperm chromatin stability. International Journal of Andrology 13(4):245-252. doi: 10.1111/j.1365-2605.1990.tb01028.x

Labas, V., L. Spina, C. Belleannee, A. P. Teixeira-Gomes, A. Gargaros, F. Dacheux, and J. L. Dacheux. 2015. Analysis of epididymal sperm maturation by MALDI profiling and top-down mass spectrometry. Journal of proteomics 113:226-243. doi: 10.1016/j.jprot.2014.09.031 
Lacorte, L. M., J. C. Rinaldi, L. A. Justulin, Jr., F. K. Delella, A. Moroz, and S. L. Felisbino. 2015. Cadmium exposure inhibits MMP2 and MMP9 activities in the prostate and testis. Biochemical and biophysical research communications 457(4):538-541. doi: 10.1016/j.bbrc.2015.01.019

Lavon, U., and J. C. Boursnel. 1975. The split ejaculate of the boar: Contributions of the epidiymides and seminal vesicles. J. Reprod. Fert. 42(3):541. doi: $10.1530 /$ jrf.0.0420541

Leclerc, P., E. de Lamirande, and C. Gagnon. 1996. Cyclic adenosine $3^{\prime}, 5^{\prime}$ monophosphate-dependent regulation of protein tyrosine phosphorylation in relation to human sperm capacitation and motility. Biology of reproduction 55(3):684-692.

Lee, S. R. 2018a. Critical Role of Zinc as Either an Antioxidant or a Prooxidant in Cellular Systems. Oxidative Medicine and Cellular Longevity 2018:11. doi: $10.1155 / 2018 / 9156285$

Lee, S. R. 2018b. Critical Role of Zinc as Either an Antioxidant or a Prooxidant in Cellular Systems. Oxid Med Cell Longev 2018:9156285. doi: $10.1155 / 2018 / 9156285$

Leemans, B., B. M. Gadella, T. A. Stout, H. Nelis, M. Hoogewijs, and A. Van Soom. 2015. An alkaline follicular fluid fraction induces capacitation and limited release of oviduct epithelium-bound stallion sperm. Reproduction 150(3):193-208. doi: 10.1530/rep-15-0178 
Lefebvre, R., and S. S. Suarez. 1996. Effect of capacitation on bull sperm binding to homologous oviductal epithelium. Biology of reproduction 54(3):575582.

Leitzmann, M. F., M. J. Stampfer, K. Wu, G. A. Colditz, W. C. Willett, and E. L. Giovannucci. 2003. Zinc Supplement Use and Risk of Prostate Cancer. JNCI: Journal of the National Cancer Institute 95(13):1004-1007. doi: 10.1093/jnci/95.13.1004

Levaot, N., and M. Hershfinkel. 2018. How cellular Zn(2+) signaling drives physiological functions. Cell Calcium 75:53-63. doi: S01434160(18)30116-7 [pii]

10.1016/j.ceca.2018.08.004

Lichten, L. A., and R. J. Cousins. 2009. Mammalian zinc transporters: nutritional and physiologic regulation. Annual review of nutrition 29:153-176. doi: 10.1146/annurev-nutr-033009-083312

Lippa, E. 1991. The eye: topical carbonic anhydrase inhibitors. In: S. Dodgson, R. Tashian, G. Gros and N. Carter, editors, The Carbonic Anhydrases: Cellular Physiology and Molecular Genetics. Plenum Press, New York. p. $171-182$.

Lishko, P. V., I. L. Botchkina, A. Fedorenko, and Y. Kirichok. 2010. Acid extrusion from human spermatozoa is mediated by flagellar voltage-gated proton channel. Cell 140(3):327-337. doi: 10.1016/j.cell.2009.12.053 
Lishko, P. V., and Y. Kirichok. 2010. The role of Hv1 and CatSper channels in sperm activation. J Physiol 588(Pt 23):4667-4672. doi:

10.1113/jphysiol.2010.194142

Liu, D.-Y., B.-S. Sie, M.-L. Liu, F. Agresta, and H. G. Baker. 2009. Relationship between seminal plasma zinc concentration and spermatozoa-zona pellucida binding and the ZP-induced acrosome reaction in subfertile men. 11(4):499-507. doi: 10.1038/aja.2009.23

Llobet, J. M., J. L. Domingo, M. T. Colomina, E. Mayayo, and J. Corbella. 1988. Subchronic oral toxicity of zinc in rats. Bulletin of environmental contamination and toxicology 41(1):36-43.

Loeb, J. 1914. On Some Non-Specific Factors for the Entrance of the Spermatozoon into the Egg. Science (New York, N.Y.) 40(1026):316-318.

Lopez-Gonzalez, I., C. L. Trevino, and A. Darszon. 2016. Regulation of Spermatogenic Cell T-Type $\mathrm{Ca}(2+)$ Currents by $\mathrm{Zn}(2+)$ : Implications in Male Reproductive Physiology. Journal of cellular physiology 231(3):659667. doi: $10.1002 / j c p .25112$

Lovell, M. A. 2009. A potential role for alterations of zinc and zinc transport proteins in the progression of Alzheimer's disease. Journal of Alzheimer's disease : JAD 16(3):471-483. doi: 10.3233/jad-2009-0992

Lu, C., R. Lee, Y. Hwu, S. Chu, Y. Chen, W. Chang, S. Lin, and S. Li. 2011. SERPINE2, a serine protease inhibitor extensively expressed in adult male mouse reproductive tissues, may serve as a murine sperm 
decapacitation factor. Biology of reproduction 84(3):514-525. doi:

10.1095/biolreprod. 110.085100

Luise, C., M. Capra, M. Donzelli, G. Mazzarol, M. G. Jodice, P. Nuciforo, G. Viale, P. P. Di Fiore, and S. Confalonieri. 2011. An atlas of altered expression of deubiquitinating enzymes in human cancer. PloS one 6(1):e15891. doi: 10.1371/journal.pone.0015891

Luque, G. M., T. Dalotto-Moreno, D. Martin-Hidalgo, C. Ritagliati, L. C. Puga Molina, A. Romarowski, P. A. Balestrini, L. J. Schiavi-Ehrenhaus, N. Gilio, D. Krapf, P. E. Visconti, and M. G. Buffone. 2018. Only a subpopulation of mouse sperm displays a rapid increase in intracellular calcium during capacitation. Journal of cellular physiology doi: 10.1002/jcp.26883

Mahajan, S. K., A. S. Prasad, and F. D. McDonald. 1984. Sexual dysfunction in uremic male: improvement following oral zinc supplementation. Contributions to nephrology 38:103-111.

Mankad, M., N. G. Sathawara, H. Doshi, H. N. Saiyed, and S. Kumar. 2006. Seminal plasma zinc concentration and alpha-glucosidase activity with respect to semen quality. Biological trace element research 110(2):97106. doi: $10.1385 /$ bter:110:2:97

Manzo, S., S. Schiavo, M. Oliviero, A. Toscano, M. Ciaravolo, and P. Cirino. 2017. Immune and reproductive system impairment in adult sea urchin exposed to nanosized $\mathrm{ZnO}$ via food. The Science of the total environment 599-600:9-13. doi: 10.1016/j.scitotenv.2017.04.173 
Maret, W., and A. Krezel. 2007. Cellular zinc and redox buffering capacity of metallothionein/thionein in health and disease. Molecular medicine (Cambridge, Mass.) 13(7-8):371-375. doi: 10.2119/2007-00036.Maret Marklund, S. L. 1984. Extracellular superoxide dismutase and other superoxide dismutase isoenzymes in tissues from nine mammalian species. The Biochemical journal 222(3):649-655.

Marti, E., L. Mara, J. I. Marti, T. Muino-Blanco, and J. A. Cebrian-Perez. 2007. Seasonal variations in antioxidant enzyme activity in ram seminal plasma. Theriogenology 67(9):1446-1454. doi: 10.1016/j.theriogenology.2007.03.002

Mason, G. G., K. B. Hendil, and A. J. Rivett. 1996. Phosphorylation of proteasomes in mammalian cells. Identification of two phosphorylated subunits and the effect of phosphorylation on activity. European journal of biochemistry / FEBS 238(2):453-462.

Massanyi, P., J. Trandzik, P. Nad, B. Korenekova, M. Skalicka, R. Toman, N. Lukac, P. Strapak, M. Halo, and J. Turcan. 2003. Concentration of copper, iron, zinc, cadmium, lead, and nickel in boar semen and relation to the spermatozoa quality. Journal of environmental science and health. Part A, Toxic/hazardous substances \& environmental engineering 38(11):26432651. doi: 10.1081/ESE-120024453

Matsumura, K., and K. Aketa. 1991. Proteasome (multicatalytic proteinase) of sea urchin sperm and its possible participation in the acrosome reaction. Mol Reprod Dev 29(2):189-199. doi: 10.1002/mrd.1080290215 
McCall, K. A., C.-C. Huang, and C. A. Fierke. 2000. Function and Mechanism of Zinc Metalloenzymes. The Journal of nutrition 130(5):1437S-1446S. doi: $10.1093 / \mathrm{jn} / 130.5 .1437 \mathrm{~s}$

McCord, J. M., and I. Fridovich. 1969. Superoxide dismutase. An enzymic function for erythrocuprein (hemocuprein). The Journal of biological chemistry 244(22):6049-6055.

Ménézo, Y., L. Pluntz, J. Chouteau, T. Gurgan, A. Demirol, A. Dalleac, and M. Benkhalifa. 2011. Zinc concentrations in serum and follicular fluid during ovarian stimulation and expression of $\mathrm{Zn} 2+$ transporters in human oocytes and cumulus cells. Reproductive biomedicine online 22(6):647-652. doi: http://dx.doi.org/10.1016/j.rbmo.2011.03.015

Merrells, K. J., H. Blewett, J. A. Jamieson, C. G. Taylor, and M. Suh. 2009. Relationship between abnormal sperm morphology induced by dietary zinc deficiency and lipid composition in testes of growing rats. The British journal of nutrition 102(2):226-232. doi: 10.1017/s0007114508159037

Michailov, Y., D. Ickowicz, and H. Breitbart. 2014. Zn2+-stimulation of sperm capacitation and of the acrosome reaction is mediated by EGFR activation. Dev Biol 396(2):246-255. doi: 10.1016/j.ydbio.2014.10.009 Mildvan, A. S., R. D. Kobes, and W. J. Rutter. 1971. Magnetic resonance studies of the role of the divalent cation in the mechanism of yeast aldolase. Biochemistry 10(7):1191-1204.

Miles, E. L., C. O'Gorman, J. Zhao, M. Samuel, E. Walters, Y. J. Yi, M. Sutovsky, R. S. Prather, K. D. Wells, and P. Sutovsky. 2013. Transgenic pig carrying 
green fluorescent proteasomes. Proc Natl Acad Sci U S A 110(16):63346339. doi: 10.1073/pnas.1220910110

Miller, D. J. 2015. Regulation of sperm function by oviduct fluid and the epithelium: Insight into the role of glycans. Reprod Domest Anim 50 Suppl 2:31-39. doi: 10.1111/rda.12570

Miller, M. R., S. J. Kenny, N. Mannowetz, S. A. Mansell, M. Wojcik, S. Mendoza, R. S. Zucker, K. Xu, and P. V. Lishko. 2018. Asymmetrically Positioned Flagellar Control Units Regulate Human Sperm Rotation. Cell reports 24(10):2606-2613. doi: 10.1016/j.celrep.2018.08.016

Mochida, K., L. L. Tres, and A. L. Kierszenbaum. 2000. Structural features of the $26 \mathrm{~S}$ proteasome complex isolated from rat testis and sperm tail. Mol Reprod Dev 57(2):176-184. doi: 10.1002/10982795(200010)57:2<176::AID-MRD9>3.0.CO;2-O [pii] 10.1002/1098-2795(200010)57:2<176::AID-MRD9>3.0.CO;2-O Mogielnicka-Brzozowska, M., R. Strzezek, K. Wasilewska, and W. Kordan. 2015. Prostasomes of canine seminal plasma - zinc-binding ability and effects on motility characteristics and plasma membrane integrity of spermatozoa. Reproduction in domestic animals = Zuchthygiene 50(3):484-491. doi: 10.1111/rda.12516

Mogielnicka-Brzozowska, M., P. Wysocki, J. Strzezek, and W. Kordan. 2011. Zinc-binding proteins from boar seminal plasma -- isolation, biochemical characteristics and influence on spermatozoa stored at 4 degrees C. Acta biochimica Polonica 58(2):171-177. 
Morales, P., E. S. Diaz, and M. Kong. 2007. Proteasome activity and its relationship with protein phosphorylation during capacitation and acrosome reaction in human spermatozoa. Society of Reproduction and Fertility supplement 65:269-273.

Morales, P., M. Kong, E. Pizarro, and C. Pasten. 2003. Participation of the sperm proteasome in human fertilization. Human reproduction 18(5):1010-1017.

Mori, E., H. Fukuda, S. Imajoh-Ohmi, T. Mori, and S. Takasaki. 2012. Purification of $\mathrm{N}$-acetyllactosamine-binding activity from the porcine sperm membrane: possible involvement of an ADAM complex in the carbohydrate-binding activity of sperm. J Reprod Dev 58(1):117-125.

Nagase, H., R. Visse, and G. Murphy. 2006. Structure and function of matrix metalloproteinases and TIMPs. Cardiovascular research 69(3):562-573. doi: 10.1016/j.cardiores.2005.12.002

Nagase, H., and J. F. Woessner, Jr. 1999. Matrix metalloproteinases. The Journal of biological chemistry 274(31):21491-21494. doi: 10.1074/jbc.274.31.21491

Nanassy, L., L. Liu, J. Griffin, and D. T. Carrell. 2011. The clinical utility of the protamine 1/protamine 2 ratio in sperm. Protein and peptide letters 18(8):772-777. doi: 10.2174/092986611795713934

Narasimhaiah, M., A. Arunachalam, S. Sellappan, V. K. Mayasula, P. R. Guvvala, S. K. Ghosh, V. Chandra, J. Ghosh, and H. Kumar. 2018. Organic zinc and copper supplementation on antioxidant protective mechanism and their correlation with sperm functional characteristics in 
goats. Reproduction in domestic animals = Zuchthygiene 53(3):644-654. doi: $10.1111 /$ rda. 13154

Nenkova, G., L. Petrov, and A. Alexandrova. 2017. Role of Trace Elements for Oxidative Status and Quality of Human Sperm. Balkan medical journal doi: 10.4274/balkanmedj.2016.0147

Nixon, B., D. A. Maclntyre, L. A. Mitchell, G. M. Gibbs, M. O'Bryan, and R. J. Aitken. 2006. The identification of mouse sperm-surface-associated proteins and characterization of their ability to act as decapacitation factors. Biology of reproduction 74(2):275-287. doi:

10.1095/biolreprod.105.044644

Noguchi, S., Y. Hatanaka, T. Tobita, and M. Nakano. 1992. Structural analysis of the N-linked carbohydrate chains of the 55-kDa glycoprotein family (PZP3) from porcine zona pellucida. European journal of biochemistry / FEBS 204(3):1089-1100. doi: doi.org/10.1111/j.1432-1033.1992.tb16733.x

Noguchi, S., and M. Nakano. 1992. Structure of the acidic N-linked carbohydrate chains of the 55-kDa glycoprotein family (PZP3) from porcine zona pellucida. European Journal of Biochemistry 209(3):883-894. doi: 10.1111/j.1432-1033.1992.tb17361.x

O'Flaherty, C., M. Beconi, and N. Beorlegui. 1997. Effect of natural antioxidants, superoxide dismutase and hydrogen peroxide on capacitation of frozenthawed bull spermatozoa. Andrologia 29(5):269-275. 
O'Flaherty, C., and D. M. Fournier. 2017. Reactive oxygen species and protein modifications in spermatozoa. Biology of reproduction doi: 10.1093/biolre/iox104

Outten, C. E., and T. V. O'Halloran. 2001. Femtomolar sensitivity of metalloregulatory proteins controlling zinc homeostasis. Science (New York, N.Y.) 292(5526):2488-2492. doi: 10.1126/science.1060331

Pacey, A. A., N. Davies, M. A. Warren, C. L. Barratt, and I. D. Cooke. 1995. Hyperactivation may assist human spermatozoa to detach from intimate association with the endosalpinx. Human reproduction 10(10):2603-2609.

Park, K., S. Jeon, Y.-J. Song, and L. S. H. Yi. 2012. Proteomic analysis of boar spermatozoa and quantity changes of superoxide dismutase 1 , glutathione peroxidase, and peroxiredoxin 5 during epididymal maturation. Animal Reproduction Science 135(1):53-61. doi: https://doi.org/10.1016/j.anireprosci.2012.08.027

Pasten, C., P. Morales, and M. Kong. 2005. Role of the sperm proteasome during fertilization and gamete interaction in the mouse. Mol Reprod Dev 71(2):209-219. doi: 10.1002/mrd.20280

Pastor-Soler, N., V. Beaulieu, T. N. Litvin, N. Da Silva, Y. Chen, D. Brown, J. Buck, L. R. Levin, and S. Breton. 2003. Bicarbonate-regulated adenylyl cyclase (sAC) is a sensor that regulates $\mathrm{pH}$-dependent V-ATPase recycling. The Journal of biological chemistry 278(49):49523-49529. doi: 10.1074/jbc.M309543200 
Patek, E., and K. Hagenfeldt. 1974. Trace elements in the human fallopian tube epithelium. Copper, zinc, manganese and potassium in the menstrual cycle. International journal of fertility 19(2):85-88.

Peeker, R., L. Abramsson, and S. L. Marklund. 1997. Superoxide dismutase isoenzymes in human seminal plasma and spermatozoa. Molecular human reproduction 3(12):1061-1066.

Petrunkina, A., A. Lakamp, M. Gentzel, M. Ekhlasi-Hundrieser, and E. TopferPetersen. 2003. Fate of lactadherin P47 during post-testicular maturation and capacitation of boar spermatozoa. Reproduction 125(3):377-387. doi: 10.1530/rep.0.1250377

Platt, M. D., A. M. Salicioni, D. F. Hunt, and P. E. Visconti. 2009. Use of Differential Isotopic Labeling and Mass Spectrometry To Analyze Capacitation-Associated Changes in the Phosphorylation Status of Mouse Sperm Proteins. Journal of proteome research 8(3):1431-1440. doi: $10.1021 / p r 800796 j$

Plum, L. M., L. Rink, and H. Haase. 2010. The essential toxin: impact of zinc on human health. International journal of environmental research and public health 7(4):1342-1365. doi: 10.3390/ijerph7041342

Porath, J., J. Carlsson, I. Olsson, and G. Belfrage. 1975. Metal chelate affinity chromatography, a new approach to protein fractionation. Nature 258(5536):598-599.

Posthuma, L., R. Baerselman, R. P. M. Van Veen, and E. M. Dirven-Van Breemen. 1997. Single and Joint Toxic Effects of Copper and Zinc on 
Reproduction ofEnchytraeus crypticusin Relation to Sorption of Metals in Soils. Ecotoxicology and Environmental Safety 38(2):108-121. doi: https://doi.org/10.1006/eesa.1997.1568

Powell, S. R. 2000. The antioxidant properties of zinc. The Journal of nutrition 130(5S Suppl):1447s-1454s. doi: 10.1093/jn/130.5.1447S

Prakash, A., K. Bharti, and A. B. Majeed. 2015. Zinc: indications in brain disorders. Fundamental \& clinical pharmacology 29(2):131-149. doi: $10.1111 / \mathrm{fcp} .12110$

Prasad, A. S. 2009. Zinc: role in immunity, oxidative stress and chronic inflammation. Curr Opin Clin Nutr Metab Care 12(6):646-652. doi: 10.1097/MCO.0b013e3283312956

Prasad, A. S. 2013. Discovery of human zinc deficiency: its impact on human health and disease. Adv Nutr 4(2):176-190. doi: 4/2/176

10.3945/an.112.003210

Prasad, A. S., C. S. Mantzoros, F. W. Beck, J. W. Hess, and G. J. Brewer. 1996. Zinc status and serum testosterone levels of healthy adults. Nutrition (Burbank, Los Angeles County, Calif.) 12(5):344-348.

Prasad, A. S., and D. Oberleas. 1970. Binding of zinc to amino acids and serum proteins in vitro. The Journal of laboratory and clinical medicine 76(3):416425.

Pursel, V. G., and L. A. Johnson. 1975. Freezing of boar spermatozoa: fertilizing capacity with concentrated semen and a new thawing procedure. Journal of animal science 40(1):99-102. 
Qi, H., M. M. Moran, B. Navarro, J. A. Chong, G. Krapivinsky, L. Krapivinsky, Y. Kirichok, I. S. Ramsey, T. A. Quill, and D. E. Clapham. 2007. All four CatSper ion channel proteins are required for male fertility and sperm cell hyperactivated motility. Proc Natl Acad Sci U S A 104(4):1219-1223. doi: 10.1073/pnas.0610286104

Qin, Y., P. J. Dittmer, J. G. Park, K. B. Jansen, and A. E. Palmer. 2011. Measuring steady-state and dynamic endoplasmic reticulum and Golgi Zn2+ with genetically encoded sensors. Proc Natl Acad Sci U S A 108(18):7351-7356. doi: 10.1073/pnas.1015686108

Que, E. L., R. Bleher, F. E. Duncan, B. Y. Kong, S. C. Gleber, S. Vogt, S. Chen, S. A. Garwin, A. R. Bayer, V. P. Dravid, T. K. Woodruff, and T. V. O'Halloran. 2015. Quantitative mapping of zinc fluxes in the mammalian egg reveals the origin of fertilization-induced zinc sparks. Nat Chem 7(2):130-139. doi: 10.1038/nchem.2133

Que, E. L., F. E. Duncan, A. R. Bayer, S. J. Philips, E. W. Roth, R. Bleher, S. C. Gleber, S. Vogt, T. K. Woodruff, and T. V. O'Halloran. 2017. Zinc sparks induce physiochemical changes in the egg zona pellucida that prevent polyspermy. Integrative biology : quantitative biosciences from nano to macro 9(2):135-144. doi: 10.1039/c6ib00212a

Rabouille, C., T. Misteli, R. Watson, and G. Warren. 1995. Reassembly of Golgi stacks from mitotic Golgi fragments in a cell-free system. The Journal of cell biology 129(3):605-618. 
Rawe, V. Y., E. S. Diaz, R. Abdelmassih, C. Wojcik, P. Morales, P. Sutovsky, and H. E. Chemes. 2008. The role of sperm proteasomes during sperm aster formation and early zygote development: implications for fertilization failure in humans. Human reproduction (Oxford, England) 23(3):573-580. doi: 10.1093/humrep/dem385

Redgrove, K. A., A. L. Anderson, M. D. Dun, E. A. McLaughlin, M. K. O'Bryan, R. J. Aitken, and B. Nixon. 2011. Involvement of multimeric protein complexes in mediating the capacitation-dependent binding of human spermatozoa to homologous zonae pellucidae. Dev Biol 356(2):460-474. doi: 10.1016/j.ydbio.2011.05.674

Reeves, P. G., and B. L. O'Dell. 1988. Zinc deficiency in rats and angiotensinconverting enzyme activity: comparative effects on lung and testis. The Journal of nutrition 118(5):622-626. doi: 10.1093/jn/118.5.622

Riffo, M., S. Leiva, and J. Astudillo. 1992. Effect of zinc on human sperm motility and the acrosome reaction. Int J Androl 15(3):229-237.

Robert, M., and C. Gagnon. 1999. Semenogelin I: a coagulum forming, multifunctional seminal vesicle protein. Cellular and molecular life sciences : CMLS 55(6-7):944-960.

Robertson, S. A. 2005. Seminal plasma and male factor signalling in the female reproductive tract. Cell and Tissue Research 322(1):43-52. doi: $10.1007 / \mathrm{s} 00441-005-1127-3$

Rodriguez-Martinez, H., U. Kvist, F. Saravia, M. Wallgren, A. Johannisson, L. Sanz, F. J. Pena, E. A. Martinez, J. Roca, J. M. Vazquez, and J. J. 
Calvete. 2009. The physiological roles of the boar ejaculate. Soc Reprod Fertil Suppl 66:1-21.

Rodriguez-Martinez, H., F. Saravia, M. Wallgren, J. Roca, and F. J. Pena. 2008.

Influence of seminal plasma on the kinematics of boar spermatozoa during freezing. Theriogenology 70(8):1242-1250. doi:

10.1016/j.theriogenology.2008.06.007

Rodriguez-Miranda, E., M. G. Buffone, S. E. Edwards, T. S. Ord, K. Lin, M. D.

Sammel, G. L. Gerton, S. B. Moss, and C. J. Williams. 2008. Extracellular adenosine $5^{\prime}$-triphosphate alters motility and improves the fertilizing capability of mouse sperm. Biology of reproduction 79(1):164-171. doi:

biolreprod.107.065565 [pii]

10.1095/biolreprod.107.065565

Rogers, J., and R. Yanagimachi. 1975. Release of hyaluronidase from guinea-pig spermatozoa through an acrosome reaction initiated by calcium. J Reprod Fertil 44(1):135-138.

Roomans, G. M., E. Lundevall, L. Bjorndahl, and U. Kvist. 1982. Removal of zinc from subcellular regions of human spermatozoa by EDTA treatment studied by X-ray microanalysis. Int J Androl 5(5):478-486.

Rosales, O., C. Opazo, E. S. Diaz, J. V. Villegas, R. Sanchez, and P. Morales. 2011. Proteasome activity and proteasome subunit transcripts in human spermatozoa separated by a discontinuous Percoll gradient. Andrologia 43(2):106-113. doi: 10.1111/j.1439-0272.2009.01029.x 
Rukgauer, M., J. Klein, and J. D. Kruse-Jarres. 1997. Reference values for the trace elements copper, manganese, selenium, and zinc in the serum/plasma of children, adolescents, and adults. Journal of trace elements in medicine and biology : organ of the Society for Minerals and Trace Elements (GMS) 11(2):92-98. doi: 10.1016/s0946-672x(97)80032-6

Saengsoi, W., W. Y. Shia, C. L. Shyu, J. T. Wu, C. Warinrak, W. M. Lee, and F. P. Cheng. 2011. Detection of matrix metalloproteinase (MMP)-2 and MMP-9 in canine seminal plasma. Animal reproduction science 127(12):114-119. doi: 10.1016/j.anireprosci.2011.07.004

Saitoh, Y., H. Sawada, and H. Yokosawa. 1993. High-molecular-weight protease complexes (proteasomes) of sperm of the ascidian, Halocynthia roretzi: isolation, characterization, and physiological roles in fertilization. Dev Biol 158(1):238-244. doi: 10.1006/dbio.1993.1182

S0012-1606(83)71182-6 [pii]

Sakai, N., H. Sawada, and H. Yokosawa. 2003. Extracellular ubiquitin system implicated in fertilization of the ascidian, Halocynthia roretzi: isolation and characterization. Dev Biol 264(1):299-307. doi: S0012160603004500 [pii] Saldívar-Hernández, A., M. E. González-González, A. Sánchez-Tusié, I. Maldonado-Rosas, P. López, C. L. Treviño, F. Larrea, and M. Chirinos. 2015. Human sperm degradation of zona pellucida proteins contributes to fertilization. Reproductive biology and endocrinology : RB\&E 13(1):99. doi: $10.1186 / \mathrm{s} 12958-015-0094-0$ 
Sánchez, R., M. Deppe, M. Schulz, P. Bravo, J. Villegas, P. Morales, and J. Risopatrón. 2011. Participation of the sperm proteasome during in vitro fertilisation and the acrosome reaction in cattle. Andrologia 43(2):114-120. doi: 10.1111/j.1439-0272.2009.01031.x

Sawada, H., H. Yokosawa, T. Someno, T. Saino, and S. Ishii. 1984. Evidence for the participation of two sperm proteases, spermosin and acrosin, in fertilization of the ascidian, Halocynthia roretzi: inhibitory effects of leupeptin analogs on enzyme activities and fertilization. Dev Biol 105(1):246-249.

Schmid, S., H. Henning, A. M. Petrunkina, K. F. Weitze, and D. Waberski. 2013. Response to capacitating stimuli indicates extender-related differences in boar sperm function12. Journal of animal science 91(10):5018-5025. doi: $10.2527 /$ jas.2013-6287

Scott, B. J., and A. R. Bradwell. 1983. Identification of the serum binding proteins for iron, zinc, cadmium, nickel, and calcium. Clinical chemistry 29(4):629633.

Seiler, H. G., and H. Sigel. 1988. Handbook on toxicity of inorganic compounds. Marcel Dekker, United States.

Sensi, S. L., L. M. Canzoniero, S. P. Yu, H. S. Ying, J. Y. Koh, G. A. Kerchner, and D. W. Choi. 1997. Measurement of intracellular free zinc in living cortical neurons: routes of entry. The Journal of neuroscience : the official journal of the Society for Neuroscience 17(24):9554-9564. 
Sharif, M., K. Kerns, P. Sutovsky, and D. J. Miller. 2017. Proteasomal activity is necessary for porcine sperm release from immobilzed oviduct glycans. In: Society for the Study of Reproduction, Washington, D.C.

Shihan, M., K. H. Chan, L. Konrad, and G. Scheiner-Bobis. 2015. Non-classical testosterone signaling in spermatogenic GC-2 cells is mediated through ZIP9 interacting with Gnalpha11. Cellular signalling 27(10):2077-2086. doi: 10.1016/j.cellsig.2015.07.013

Shimokawa Ki, K., M. Katayama, Y. Matsuda, H. Takahashi, I. Hara, H. Sato, and S. Kaneko. 2002. Matrix metalloproteinase (MMP)-2 and MMP-9 activities in human seminal plasma. Molecular human reproduction 8(1):32-36.

Shukla, K. K., A. A. Mahdi, and S. Rajender. 2012. Ion Channels in Sperm Physiology and Male Fertility and Infertility. Journal of Andrology 33(5):777-788. doi: 10.2164/jandrol.111.015552

Siciliano, L., C. De Stefano, M. F. Petroni, A. Vivacqua, V. Rago, and A. Carpino. 2000. Prostatic origin of a zinc binding high molecular weight protein complex in human seminal plasma. Molecular human reproduction 6(3):215-218.

Sikka, S. C. 2001. Relative impact of oxidative stress on male reproductive function. Current medicinal chemistry 8(7):851-862.

Singh, U. S., M. V. Kumar, and J. N. Panda. 1985. Angiotensin converting enzyme in semen and its possible role in capacitation. Andrologia 17(5):472-475. 
Smith, T. T., and R. Yanagimachi. 1991. Attachment and release of spermatozoa from the caudal isthmus of the hamster oviduct. J Reprod Fertil 91(2):567573.

Song, C. Y., B. Gao, H. Wu, X. Y. Wang, G. H. Chen, and J. Mao. 2010. Spatial and temporal expression of spermadhesin genes in reproductive tracts of male and female pigs and ejaculated sperm. Theriogenology 73(5):551559. doi: 10.1016/j.theriogenology.2009.09.030

Sørensen, M. B., M. Stoltenberg, G. Danscher, and E. Ernst. 1999. Chelation of intracellular zinc ions affects human sperm cell motility. MHR: Basic science of reproductive medicine 5(4):338-341. doi:

10.1093/molehr/5.4.338

Soucek, D. A., and J. C. Vary. 1984. Some properties of acid and alkaline phosphates from boar sperm plasma membranes. Biology of reproduction 31(4):687-693.

Stephenson, J. L., and B. G. Brackett. 1999. Influences of zinc on fertilisation and development of bovine oocytes in vitro. Zygote 7(3):195-201. doi: $10.1017 / \mathrm{S} 096719949900057 x$

Steven, F. S., M. M. Griffin, and E. N. Chantler. 1982. Inhibition of human and bovine sperm acrosin by divalent metal ions. Possible role of zinc as a regulator of acrosin activity. Int J Androl 5(4):401-412. doi: 10.1111/j.13652605.1982.tb00270.x

Stoltenberg, M., M. Sørensen, and G. Danscher. 1997. Histochemical demonstration of zinc ions in ejaculated human semen. International 
journal of andrology 20(4):229-236. doi: 10.1046/j.13652605.1997.00060.x

Strzezek, R., and L. Fraser. 2009. Characteristics of spermatozoa of whole ejaculate and sperm-rich fraction of dog semen following exposure to media varying in osmolality. Reproductive biology 9(2):113-126.

Suarez, S. S. 2008. Regulation of sperm storage and movement in the mammalian oviduct. Int J Dev Biol 52(5-6):455-462. doi: $10.1387 / \mathrm{ijdb} .072527 \mathrm{ss}$

Suarez, S. S. 2015. Mammalian sperm interactions with the female reproductive tract. Cell Tissue Res 10.1007/s00441-015-2244-2doi: 10.1007/s00441015-2244-2

Sugihara, T., R. Wadhwa, S. C. Kaul, and Y. Mitsui. 1999. A novel testis-specific metallothionein-like protein, tesmin, is an early marker of male germ cell differentiation. Genomics 57(1):130-136. doi: 10.1006/geno.1999.5756

Sutovsky, P. 2011. Sperm proteasome and fertilization. Reproduction 142(1):114. doi: 10.1530/REP-11-0041

Sutovsky, P., G. Manandhar, T. C. McCauley, J. N. Caamano, M. Sutovsky, W. E. Thompson, and B. N. Day. 2004. Proteasomal interference prevents zona pellucida penetration and fertilization in mammals. Biology of reproduction 71(5):1625-1637. doi: 10.1095/biolreprod.104.032532

Sutovsky, P., T. C. McCauley, M. Sutovsky, and B. N. Day. 2003. Early degradation of paternal mitochondria in domestic pig (Sus scrofa) is prevented by selective proteasomal inhibitors lactacystin and MG132. 
Biology of reproduction 68(5):1793-1800. doi:

10.1095/biolreprod.102.012799

biolreprod.102.012799 [pii]

Szewczyk, B. 2013. Zinc homeostasis and neurodegenerative disorders.

Frontiers in aging neuroscience 5:33. doi: 10.3389/fnagi.2013.00033

Talevi, R., V. Barbato, I. Fiorentino, S. Braun, S. Longobardi, and R. Gualtieri. 2013. Protective effects of in vitro treatment with zinc, d-aspartate and coenzyme q10 on human sperm motility, lipid peroxidation and DNA fragmentation. Reproductive Biology \& Endocrinology 11(1):1-10. (Article) doi: 10.1186/1477-7827-11-81

Tapiero, H., and K. D. Tew. 2003. Trace elements in human physiology and pathology: zinc and metallothioneins. Biomedicine \& pharmacotherapy $=$ Biomedecine \& pharmacotherapie 57(9):399-411.

Tardif, S., C. Dube, and J. L. Bailey. 2003. Porcine sperm capacitation and tyrosine kinase activity are dependent on bicarbonate and calcium but protein tyrosine phosphorylation is only associated with calcium. Biology of reproduction 68(1):207-213. doi: 10.1095/biolreprod.102.005082

Tardif, S., C. Dube, S. Chevalier, and J. L. Bailey. 2001. Capacitation is associated with tyrosine phosphorylation and tyrosine kinase-like activity of pig sperm proteins. Biology of reproduction 65(3):784-792. doi: 10.1095/biolreprod65.3.784

Tentes, I., B. Asimakopoulos, E. Mourvati, K. Diedrich, S. Al-Hasani, and N. Nikolettos. 2007. Matrix metalloproteinase (MMP)-2 and MMP-9 in 
seminal plasma. J Assist Reprod Genet 24(7):278-281. doi:

$10.1007 / \mathrm{s} 10815-007-9129-6$

Underwood, E., and M. Somers. 1969. Studies of zinc nutrition in sheep. I. The relation of zinc to growth, testicular development, and spermatogenesis in young rams. Australian Journal of Agricultural Research 20(5):889-897. doi: https://doi.org/10.1071/AR9690889

UniProt Consortium, T. 2018. UniProt: the universal protein knowledgebase. Nucleic Acids Research 46(5):2699-2699. doi: 10.1093/nar/gky092

Vallee, B. L., and D. S. Auld. 1990. Zinc coordination, function, and structure of zinc enzymes and other proteins. Biochemistry 29(24):5647-5659.

Vallee, B. L., and D. S. Auld. 1992. Active zinc binding sites of zinc metalloenzymes. In: H. Birkedal-Hansen, Z. Werb, W. H and H. Van Wart, editors, Matrix Metalloproteinases and Inhibitors. Fischer, Stuttgart, Germany. p. 5.

Vallee, B. L., J. E. Coleman, and D. S. Auld. 1991. Zinc fingers, zinc clusters, and zinc twists in DNA-binding protein domains. Proc Natl Acad Sci U S A 88(3):999-1003.

Vallee, B. L., and K. H. Falchuk. 1993. The biochemical basis of zinc physiology. Physiological reviews 73(1):79-118. doi: 10.1152/physrev.1993.73.1.79

Verma, R., L. Aravind, R. Oania, W. H. McDonald, J. R. Yates, 3rd, E. V. Koonin, and R. J. Deshaies. 2002. Role of Rpn11 metalloprotease in deubiquitination and degradation by the $26 \mathrm{~S}$ proteasome. Science (New York, N.Y.) 298(5593):611-615. doi: 10.1126/science.1075898 
Vettakkorumakankav, N. N., and V. S. Ananthanarayanan. 1999. Ca2+ and Zn2+ binding properties of peptide substrates of vertebrate collagenase, MMP1. 1432(2):356-370. doi: $10.1016 / \mathrm{s} 0167-4838(99) 00111-9$

Vijayaraghavan, S., G. A. Liberty, J. Mohan, V. P. Winfrey, G. E. Olson, and D. W. Carr. 1999. Isolation and molecular characterization of AKAP110, a novel, sperm-specific protein kinase A-anchoring protein. Mol Endocrinol 13(5):705-717. doi: 10.1210/mend.13.5.0278

Vinkenborg, J. L., T. J. Nicolson, E. A. Bellomo, M. S. Koay, G. A. Rutter, and M. Merkx. 2009. Genetically encoded FRET sensors to monitor intracellular Zn2+ homeostasis. Nature methods 6(10):737-740. doi:

10.1038/nmeth.1368

Virji, N. 1985. LDH-C4 in human seminal plasma and its relationship to testicular function. I. Methodological aspects. International journal of andrology 8(3):193-200.

Visconti, P. E., J. L. Bailey, G. D. Moore, D. Pan, P. Olds-Clarke, and G. S. Kopf. 1995a. Capacitation of mouse spermatozoa. I. Correlation between the capacitation state and protein tyrosine phosphorylation. Development 121(4):1129-1137.

Visconti, P. E., G. D. Moore, J. L. Bailey, P. Leclerc, S. A. Connors, D. Pan, P. Olds-Clarke, and G. S. Kopf. 1995b. Capacitation of mouse spermatozoa. II. Protein tyrosine phosphorylation and capacitation are regulated by a cAMP-dependent pathway. Development 121(4):1139-1150. 
Vivacqua, A., L. Siciliano, M. Sabato, A. Palma, and A. Carpino. 2004.

Prostasomes as zinc ligands in human seminal plasma. International journal of andrology 27(1):27-31.

Vivet, F., P. Callard, and A. Gamoudi. 1987. Immunolocalization of angiotensin 1 converting enzyme in the human male genital tract by the avidin-biotincomplex method. Histochemistry 86(5):499-502.

Wallgren, M., F. Saravia, and H. Rodriguez-Martinez. 2010. The vanguard sperm cohort of the boar ejaculate is overrepresented in the tubal sperm reservoir in vivo. J Reprod Dev 56(1):68-72.

Wang, D., J. Hu, I. A. Bobulescu, T. A. Quill, P. McLeroy, O. W. Moe, and D. L. Garbers. 2007. A sperm-specific $\mathrm{Na}+/ \mathrm{H}+$ exchanger (sNHE) is critical for expression and in vivo bicarbonate regulation of the soluble adenylyl cyclase (sAC). Proc Natl Acad Sci U S A 104(22):9325-9330. doi: 10.1073/pnas.0611296104

Warinrak, C., J. T. Wu, W. L. Hsu, J. W. Liao, S. C. Chang, and F. P. Cheng. 2015. Expression of matrix metalloproteinases (MMP-2, MMP-9) and their inhibitors (TIMP-1, TIMP-2) in canine testis, epididymis and semen. Reproduction in domestic animals $=$ Zuchthygiene 50(1):48-57. doi: $10.1111 /$ rda. 12448

Wastney, M. E., R. L. Aamodt, W. F. Rumble, and R. I. Henkin. 1986. Kinetic analysis of zinc metabolism and its regulation in normal humans. The American journal of physiology 251(2 Pt 2):R398-408. doi:

10.1152/ajpregu.1986.251.2.R398 
Wellenreuther, G., M. Cianci, R. Tucoulou, W. Meyer-Klaucke, and H. Haase. 2009. The ligand environment of zinc stored in vesicles. Biochemical and biophysical research communications 380(1):198-203. doi: 10.1016/j.bbrc.2009.01.074

Wheat, T. E., and E. Goldberg. 1983. Sperm-specific lactate dehydrogenase C4: antigenic structure and immunosuppression of fertility. In: J. G. Scandalios and G. S. Whitt, editors, Isozymes. Current topics in Biological and Medical Research. Liss, New York. p. 113-130.

Winters, R. A., L. M. Nettenstrom, D. G. Lopez, K. L. Willenburg, R. Vishwanath, N. V. Bovin, and D. J. Miller. 2018. Effect of sorting boar spermatozoa by sex chromosomes on oviduct cell binding. Theriogenology 108:22-28. doi: 10.1016/j.theriogenology.2017.11.010

Wojcik, C., M. Benchaib, J. Lornage, J. C. Czyba, and J. F. Guerin. 2000. Proteasomes in human spermatozoa. International journal of andrology 23(3):169-177.

Wroblewski, N., W.-B. Schill, and R. Henkel. 2003. Metal chelators change the human sperm motility pattern. Fertility and sterility 79:1584-1589. doi: $10.1016 / \mathrm{S} 0015-0282(03) 00255-3$

Xu, K., and H. Qi. 2014. Sperm-specific AKAP3 is a dual-specificity anchoring protein that interacts with both protein kinase a regulatory subunits via conserved N-terminal amphipathic peptides. Mol Reprod Dev 81(7):595607. doi: $10.1002 / \mathrm{mrd} .22329$ 
Yamaguchi, S., C. Miura, K. Kikuchi, F. T. Celino, T. Agusa, S. Tanabe, and T. Miura. 2009. Zinc is an essential trace element for spermatogenesis. Proceedings of the National Academy of Sciences 106(26):10859-10864. doi: 10.1073/pnas.0900602106

Yanagimachi, R. 1982. Requirement of extracellular calcium ions for various stages of fertilization and fertilization-related phenomena in the hamster. Gamete Research 5(4):323-344. doi: doi:10.1002/mrd.1120050404

Yanagimachi, R., and Y. Kanoh. 1953. Manner of Sperm Entry in Herring Egg, with Special Reference to the Role of Calcium lons in Fertilization. Journal of the Faculty of Science Hokkaido University 11(3):487.

Yanagimachi, R., and N. Usui. 1974. Calcium dependence of the acrosome reaction and activation of guinea pig spermatozoa. Experimental cell research 89(1):161-174. doi: https://doi.org/10.1016/0014-4827(74)901992

Yi, Y. J., G. Manandhar, R. J. Oko, W. G. Breed, and P. Sutovsky. 2007. Mechanism of sperm-zona pellucida penetration during mammalian fertilization: 26S proteasome as a candidate egg coat lysin. Soc Reprod Fertil Suppl 63:385-408.

Yi, Y. J., G. Manandhar, M. Sutovsky, V. Jonakova, C. S. Park, and P. Sutovsky. 2010a. Inhibition of 19S proteasomal regulatory complex subunit PSMD8 increases polyspermy during porcine fertilization in vitro. Journal of reproductive immunology 84(2):154-163. doi: 10.1016/j.jri.2009.11.002 
Yi, Y. J., G. Manandhar, M. Sutovsky, S. W. Zimmerman, V. Jonakova, F. W. van Leeuwen, R. Oko, C. S. Park, and P. Sutovsky. 2010b. Interference with the 195 proteasomal regulatory complex subunit PSMD4 on the sperm surface inhibits sperm-zona pellucida penetration during porcine fertilization. Cell Tissue Res 341(2):325-340. doi: 10.1007/s00441-0100988-2

Yi, Y. J., C. S. Park, E. S. Kim, E. S. Song, J. H. Jeong, and P. Sutovsky. 2009. Sperm-surface ATP in boar spermatozoa is required for fertilization: relevance to sperm proteasomal function. Syst Biol Reprod Med 55(2):8596. doi: $10.1080 / 19396360802699074$

Yi, Y. J., M. Sutovsky, C. Kennedy, and P. Sutovsky. 2012a. Identification of the inorganic pyrophosphate metabolizing, ATP substituting pathway in mammalian spermatozoa. PloS one 7(4):e34524. doi:

10.1371/journal.pone.0034524

Yi, Y. J., S. W. Zimmerman, G. Manandhar, J. F. Odhiambo, C. Kennedy, V. Jonakova, P. Manaskova-Postlerova, M. Sutovsky, C. S. Park, and P. Sutovsky. 2012b. Ubiquitin-activating enzyme (UBA1) is required for sperm capacitation, acrosomal exocytosis and sperm-egg coat penetration during porcine fertilization. Int J Androl 35(2):196-210. doi: 10.1111/j.13652605.2011.01217.x

Yi, Y. J., S. W. Zimmerman, G. Manandhar, J. F. Odhiambo, C. Kennedy, V. Jonáková, P. Maňásková-Postlerová, M. Sutovsky, C. S. Park, and P. Sutovsky. 2012c. Ubiquitin-activating enzyme (UBA1) is required for 
sperm capacitation, acrosomal exocytosis and sperm-egg coat penetration during porcine fertilization. International Journal of Andrology 35(2):196210. (Article) doi: 10.1111/j.1365-2605.2011.01217.x

Yokota, N., and H. Sawada. 2007. Sperm proteasomes are responsible for the acrosome reaction and sperm penetration of the vitelline envelope during fertilization of the sea urchin Pseudocentrotus depressus. Dev Biol 308(1):222-231. doi: S0012-1606(07)01049-4 [pii]

10.1016/j.ydbio.2007.05.025

Yotsumoto, H., S. Sato, and M. Shibuya. 1984. Localization of angiotensin converting enzyme (dipeptidyl carboxypeptidase) in swine sperm by immunofluorescence. Life sciences 35(12):1257-1261.

Yurewicz, E. C., B. A. Pack, and A. G. Sacco. 1991. Isolation, composition, and biological activity of sugar chains of porcine oocyte zona pellucida $55 \mathrm{~K}$ glycoproteins. Mol Reprod Dev 30(2):126-134. doi:

10.1002/mrd.1080300209

Zalewski, P. D., I. J. Forbes, and W. H. Betts. 1993. Correlation of apoptosis with change in intracellular labile $\mathrm{Zn}(\mathrm{II})$ using zinquin [(2-methyl-8-ptoluenesulphonamido-6-quinolyloxy)acetic acid], a new specific fluorescent probe for Zn(II). The Biochemical journal 296 ( Pt 2):403-408.

Zeng, Y., J. A. Oberdorf, and H. M. Florman. 1996. pH regulation in mouse

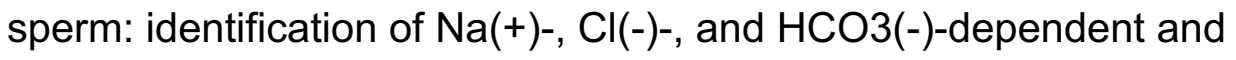
arylaminobenzoate-dependent regulatory mechanisms and 
characterization of their roles in sperm capacitation. Dev Biol 173(2):510520.

Zhang, N., F. E. Duncan, E. L. Que, T. V. O'Halloran, and T. K. Woodruff. 2016. The fertilization-induced zinc spark is a novel biomarker of mouse embryo quality and early development. Sci Rep 6:22772. doi: 10.1038/srep22772

Zhao, J., X. Dong, X. Hu, Z. Long, L. Wang, Q. Liu, B. Sun, Q. Wang, Q. Wu, and L. Li. 2016. Zinc levels in seminal plasma and their correlation with male infertility: A systematic review and meta-analysis. Sci Rep 6doi: 10.1038/srep22386

Zhao, M. H., J. W. Kwon, S. Liang, S. H. Kim, Y. H. Li, J. S. Oh, N. H. Kim, and X. S. Cui. 2014. Zinc regulates meiotic resumption in porcine oocytes via a protein kinase C-related pathway. PloS one 9(7):e102097. doi: 10.1371/journal.pone.0102097

Zhu, J., X. Xu, J. R. Cosgrove, and G. R. Foxcroft. 2000. Effects of semen plasma from different fractions of individual ejaculates on IVF in pigs. Theriogenology 54(9):1443-1452. (Article) doi: 10.1016/S0093$691 \times(00) 00466-0$

Zigo, M., V. Jonakova, M. Sulc, and P. Manaskova-Postlerova. 2013. Characterization of sperm surface protein patterns of ejaculated and capacitated boar sperm, with the detection of ZP binding candidates. International journal of biological macromolecules 61:322-328. doi: 10.1016/j.ijbiomac.2013.07.014 
Zimmerman, S. W., G. Manandhar, Y. J. Yi, S. K. Gupta, M. Sutovsky, J. F. Odhiambo, M. D. Powell, D. J. Miller, and P. Sutovsky. 2011. Sperm proteasomes degrade sperm receptor on the egg zona pellucida during mammalian fertilization. PloS one 6(2):e17256. doi: 10.1371/journal.pone.0017256

Zimmerman, S. W., Y. J. Yi, M. Sutovsky, F. W. van Leeuwen, G. Conant, and P. Sutovsky. 2014. Identification and characterization of RING-finger ubiquitin ligase UBR7 in mammalian spermatozoa. Cell Tissue Res 356(1):261-278. doi: 10.1007/s00441-014-1808-x 
VITA

Karl Kerns grew up on a swine seedstock operation in southwestern lowa. He completed his Bachelor of Science degree in Animal Science at lowa State University in 2014. During his time at lowa State, he worked as an undergraduate research assistant in the laboratory of Dr. Jason Ross. During his undergraduate career, he held many internships. These included with Trans Ova Genetics, Smithfield Premium Genetics, the USDA ARS Beltsville Animal Biosciences \& Biotechnology Laboratory, and Dr. William van Wettere's laboratory at the University of Adelaide (Adelaide, South Australia, Australia). He was awarded the University of Missouri Graduate Studies Fellowship and F.B. Miller Fellowship and began his graduate studies in the laboratory of Dr. Peter Sutovsky in August 2014. During that time, he won many awards and contests at the college and national level, with his pinnacle achievement of an USDA NIFA Predoctoral Fellowship $(\$ 95,000)$ and co-authored two provisional patents currently undergoing submission. The findings from his research have been featured in more than 18 news outlets nationally and internationally. His dissertation research focused on the role of zinc ions in regulating and managing sperm fertilization competency in boar, bull, and human in an effort to improve male fertility of livestock and humans. Pending successful dissertation defense, he will graduate in May 2019 and plans to continue post-doctoral research under the direction of Dr. Peter Sutovsky while jointly pursuing business startup opportunities based on his dissertation findings. 